\title{
Solar-like oscillations in red giants observed with Kepler: comparison of global oscillation parameters from different methods ${ }^{\star}$
}

\author{
S. Hekker ${ }^{1}$, Y. Elsworth ${ }^{1}$, J. De Ridder ${ }^{2}$, B. Mosser ${ }^{3}$, R. A. García ${ }^{4}$, T. Kallinger ${ }^{5,6}$, S. Mathur ${ }^{7}$, D. Huber ${ }^{8}$, \\ D. L. Buzasi ${ }^{9}$, H. L. Preston ${ }^{9}, 10$, S. J. Hale ${ }^{1}$, J. Ballot ${ }^{11}$, W. J. Chaplin ${ }^{1}$, C. Régulo ${ }^{12,13}$, T. R. Bedding ${ }^{8}$, D. Stello ${ }^{8}$, \\ W. J. Borucki ${ }^{14}$, D. G. Koch ${ }^{14}$, J. Jenkins ${ }^{15}$, C. Allen ${ }^{16}$, R. L. Gilliland ${ }^{17}$, H. Kjeldsen ${ }^{18}$, and J. Christensen-Dalsgaard ${ }^{18}$ \\ ${ }^{1}$ University of Birmingham, School of Physics and Astronomy, Edgbaston, Birmingham B15 2TT, UK \\ e-mail: saskia@ison.ph.bham.ac.uk \\ 2 Instituut voor Sterrenkunde, KU Leuven, Celestijnenlaan 200D, 3001 Leuven, Belgium \\ 3 LESIA, UMR8109, Université Pierre et Marie Curie, Université Denis Diderot, Observatoire de Paris, 92195 Meudon Cedex, \\ France \\ ${ }^{4}$ Laboratoire AIM, CEA/DSM-CNRS, Université Paris 7 Denis Diderot, IRFU/SAp, Centre de Saclay, 91191 Gif-sur-Yvette, France \\ 5 Department of Physics and Astronomy, University of British Colombia, 6224 Agricultural Road, Vancouver, BC V6T 1Z1, Canada \\ ${ }^{6}$ Institute for Astronomy, University of Vienna, Türkenschanzstrasse 17, 1180 Vienna, Austria \\ High Altitude Observatory, NCAR, PO Box 3000, Boulder, CO 80307, USA \\ 8 Sydney Institute for Astronomy (SIfA), School of Physics, University of Sydney, NSW 2006, Australia \\ 9 Eureka Scientific, 2452 Delmer Street Suite 100, Oakland, CA 94602-3017, USA \\ 10 Department of Mathematical Sciences, University of South Africa, Box 392, UNISA 0003, South Afrika \\ 11 Laboratoire d'Astrophysique de Toulouse-Tarbes, Université de Toulouse, CNRS, 31400 Toulouse, France \\ 12 Universidad de La Laguna, Dpto de Astrofísica, 38206 Tenerife, Spain \\ 13 Instituto de Astrofísica de Canarias, 38205 La Laguna, Tenerife, Spain \\ 14 NASA Ames Research Center, MS 244-30, Moffet Field, CA 94035, USA \\ 15 SETI Institute, NASA Ames Research Center, MS 244-30, Moffet Field, CA 94035, USA \\ 16 Orbital Sciences Corp., NASA Ames Research Center, Moffet Field, CA 94035, USA \\ 17 Space Telescope Science Institute, 3700 San Martin Drive, Baltimore, MD 21218, USA \\ 18 Department of Physics and Astronomy, Building 1520, Aarhus University, 8000 Aarhus C, Denmark
}

Received 9 June 2010 / Accepted 13 August 2010

\section{ABSTRACT}

\begin{abstract}
Context. The large number of stars for which uninterrupted high-precision photometric timeseries data are being collected with Kepler and CoRoT initiated the development of automated methods to analyse the stochastically excited oscillations in main-sequence, subgiant and red-giant stars.

Aims. We investigate the differences in results for global oscillation parameters of $\mathrm{G}$ and $\mathrm{K}$ red-giant stars due to different methods and definitions. We also investigate uncertainties originating from the stochastic nature of the oscillations.

Methods. For this investigation we use Kepler data obtained during the first four months of operation. These data have been analysed by different groups using already published methods and the results are compared. We also performed simulations to investigate the uncertainty on the resulting parameters due to different realizations of the stochastic signal.

Results. We obtain results for the frequency of maximum oscillation power $\left(v_{\max }\right)$ and the mean large separation $(\langle\Delta v\rangle)$ from different methods for over one thousand red-giant stars. The results for these parameters agree within a few percent and seem therefore robust to the different analysis methods and definitions used here. The uncertainties for $v_{\max }$ and $\langle\Delta v\rangle$ due to differences in realization noise are not negligible and should be taken into account when using these results for stellar modelling.
\end{abstract}

Key words. asteroseismology - stars: late-type - methods: observational - techniques: photometric

\section{Introduction}

Oscillations in stars provide information on their internal structures. In the Sun, oscillations are stochastically excited in the outer turbulent layer and these so-called solar-like oscillations have provided a detailed picture of its internal structure including the size of the core, the location of the tachocline and differential rotation (see for a recent overview Chaplin \& Basu 2008). With asteroseismology we observe stellar oscillations to reveal

$\star$ Table 7 is only available in electronic form at http://www . aanda.org similar information on the internal structures of stars other than the Sun.

Stars with turbulent outer layers are expected to exhibit solar-like oscillations and these are indeed observed in solar-type stars, subgiants and G-K red-giant stars. For recent overviews of these observations and interpretations see e.g., Bedding \& Kjeldsen (2008), Hekker (2010).

Currently, long time series of uninterrupted high-precision photometric data of oscillating stars are being measured by the space missions CoRoT (Convection Rotation and planetary Transits, Baglin et al. 2006) and Kepler (Borucki et al. 2009, 
see also the MAST website ${ }^{1}$ for Kepler data products available for general public). These missions have increased spectacularly the number of $\mathrm{G}$ and $\mathrm{K}$ red-giant stars in which solar-like oscillations, both radial and non-radial, are clearly detected. First results on red-giant seismology from both missions were presented by e.g. De Ridder et al. (2009), Hekker et al. (2009), Miglio et al. (2009), Carrier et al. (2010), Kallinger et al. (2010b), Mosser et al. (2010), Bedding et al. (2010), Hekker et al. (2010b), Stello et al. (2010).

In general, the time series are processed to give information on their frequency content using Fourier methods that take account of the non-uniform sampling in the time domain. When analysing these Fourier spectra, one can distinguish between an approach of detailed modelling of individual oscillation modes using Lorentz fitting (e.g., Appourchaux et al. 2008; Benomar et al. 2009; Deheuvels et al. 2010), and a more global analysis of the power spectrum which yields average seismic quantities such as the frequency of maximum power $\left(v_{\max }\right)$, the mean large frequency spacing $(\langle\Delta v\rangle)$, and a parametric fit of the granulation background. From these global seismic observables astrophysically interesting information can be extracted, such as constraints on our galaxy's starburst history (Miglio et al. 2009) and a precise determination of red giant radii and masses (e.g., Kallinger et al. 2010b).

However, as will be shown in Sect. 2, different authors use different definitions and different approximations to derive $v_{\max }$ and $\langle\Delta v\rangle$. None of these approaches can be claimed as "the" only correct one, yet there is a need to convey results to theoreticians for modelling. In this paper we quantitatively investigate to what extent the results from different approaches differ. We also study how robust are the different definitions against variations in the power spectrum due to the stochastic realization of the oscillation modes. Understanding both these sources of uncertainty in the observations is of importance for the physical interpretation of the results and seismic modelling. Finally, we discuss what can be gained by combining different methods.

The results for the global oscillation parameters of stars observed with Kepler as presented here are used for a more detailed study by Huber et al. (2010) and to investigate the asteroseismic masses and radii of the stars by Kallinger et al. (2010a).

\section{Oscillation parameters}

The particular oscillation parameters discussed in this study are the frequency of maximum oscillation power $\left(v_{\max }\right)$ and the large frequency separation $(\Delta v)$. In general, $\Delta v$ is the separation between oscillation modes with the same degree $\ell$ and consecutive radial orders $n$ and is sensitive to the sound travel time across the star. For high-order low-degree modes we expect the eigenfrequencies $\left(v_{n, \ell}\right)$ to follow approximately the asymptotic relation by Tassoul (1980), which can be expressed as:

$v_{n, \ell} \simeq \Delta v\left(n+\frac{\ell}{2}+\varepsilon\right)-\ell(\ell+1) D_{0}$,

in which $\varepsilon$ is sensitive to the surface layers and $D_{0}$ to layers deeper inside the star. Based on previous results, we take $D_{0}$ to be small compared to $\Delta v$. The large separation is approximately constant. However, it is well known from observations of the Sun and other stars that $\Delta v$ does depend on both frequency and angular degree.

\footnotetext{
${ }^{1}$ http://stdatu.stsci.edu/kepler/
}

We first describe the general methods by which we obtain $v_{\max }$ and $\langle\Delta v\rangle$. This will then be followed by a more detailed description of each method used in the present study, with the aim of investigating the similarities and differences in the resulting values obtained from different determinations.

The presence of modes due to resolved, stochasticallyexcited solar-like oscillations leads to a power excess which has a shape that can often be approximated by a Gaussian distribution. This characteristic feature is used to estimate $v_{\max }$ as the centroid of a Gaussian fitted to the (smoothed) power excess. A complexity is that the oscillations in red giants occur in a frequency regime also containing signal due to stellar activity, granulation and possibly instrumental effects. This "background" power has a slope that can influence the determination of $v_{\max }$, and must be taken into account. The background can be modeled by a sum of power laws and white noise (e.g. Harvey 1985; Hekker et al. 2009; Kallinger et al. 2010b). In some cases a simplification is valid and a linear approximation to the background over the frequency range of the oscillations is used.

The regularity in the large frequency spacing between the modes of solar-like oscillations provides a clear signature in the power spectrum of the power spectrum (PS $\otimes P S)$, which is equivalent to the autocorrelation of the timeseries. The highest features in the PS $\otimes$ PS occur at submultiples of $\Delta v$, i.e., $\Delta v / 2$, $\Delta v / 4$. Similar features from submultiples of $\Delta v$ are present in an autocorrelation of the power spectrum. Many algorithms use a rough version of the relation between $v_{\max }$ and $\Delta v$ as described by Hekker et al. (2009), Stello et al. (2009), Mosser et al. (2010) to identify which feature is due to which submultiple:

$\Delta v \sim v_{\max }^{0.73 \rightarrow 0.8}$,

in which $0.73 \rightarrow 0.8$ indicates the range of values used for the exponent. When the individual modes in the power spectrum are fitted directly, in a procedure known as peak bagging, the large separation as a function of frequency can be obtained directly. Peakbagging is only used in one approach, which is described in more detail by Kallinger et al. (2010a).

Having described the general concepts for determining $v_{\max }$ and $\langle\Delta v\rangle$, we now describe the methods used by the different teams that analysed the data. The methods are also summarised in Table 1. We refer to the references for more detailed descriptions of the methods developed by the individual teams.

- In the autocorrelation method described by Mosser \& Appourchaux (2009, hereafter COR) $v_{\max }$ is obtained as the centroid of a Gaussian fit to the smoothed power spectrum. For the smoothing, a Gaussian with full width half maximum $(F W H M)$ of $3\langle\Delta v\rangle$ is used. $\langle\Delta v\rangle$ is determined from the first peak in the autocorrelation of the timeseries apodised with a Hanning filter, where the $F W H M$ of the excess envelope is used to compute the mean value.

- In the automated Bayesian Markov-Chain Monte Carlo algorithm (Kallinger et al. 2010b; Gruberbauer et al. 2009, hereafter (AN), $v_{\max }$ is defined as the centroid of a Gaussian fitted to the unsmoothed power spectrum. $\langle\Delta v\rangle$ is obtained from peakbagging, i.e. from fitting a sequence of Lorentzian profiles spanning three radial orders to the backgroundcorrected power spectrum, parameterised by the large and small frequency separations.

- In the method described by Mathur et al. (2010, hereafter A2Z) and adapted for the analysis of red giants, $v_{\max }$ is defined from a Gaussian fit to the smoothed power spectrum. For the smoothing a boxcar of width $4\langle\Delta v\rangle$ is used. $\langle\Delta v\rangle$ is determined from the highest feature in the PS $\otimes \mathrm{PS}$, which is 
Table 1. Summary of the different methods used to determine $v_{\max }$ and $\langle\Delta v\rangle$.

\begin{tabular}{l|cccccc}
\hline \hline ID & COR & CAN & A2Z & SYD & DLB & OCT \\
\hline$v_{\max }$ & centroid Gaussian & centroid Gaussian & centroid Gaussian & peak smoothed PS & $\begin{array}{c}\text { centroid Gaussian } \\
\text { centroid Gaussian (I) } \\
\text { first moment (II) }\end{array}$ \\
smoothing & $3 \Delta v$ Gauss & none & $4 \Delta v$ box & $2 \Delta v$ Gauss & $10 \mu$ Hz Gauss & $2 \Delta v$ box \\
$\Delta v$ & autocor timeseries & fit to PS & PS $\otimes$ PS & autocor PS & autocor PS & PS $\otimes$ PS \\
freq interval & $F W H M$ osc excess & 3 radial orders & $v_{\max } \pm v_{\max } / 3$ & $v_{\max } \pm 5-7 \Delta v$ & $v_{\max } \pm v_{\max } / 4$ & osc excess \\
background & $a v^{-\beta}$ & $w+\sum_{i=1}^{3} \frac{B_{i}}{1+C_{i} v^{4}}$ & $w+\frac{B}{1+C v^{\alpha}}+a v^{-\beta}$ & $w+\sum_{i} \frac{B_{i}}{1+\left(C_{i} v\right)^{2}+\left(C_{i} v\right)^{4}}$ & $w+\frac{B}{1+C v^{2}}$ & linear approx \\
Eq. (2) & yes & no & yes & yes & yes & yes \\
\hline
\end{tabular}

Notes. We also mention the way the smoothing is applied to the power spectrum in the determination of $v_{\max }$, the frequency interval over which $\langle\Delta v\rangle$ is computed, how the background is determined and whether Eq. (2) is used. For the background we indicate the general formulae with $w$ indicating white noise, $v$ frequency, and $a, B, C, \alpha$ and $\beta$ free parameters.

computed over a range $v_{\max } \pm v_{\max } / 3$. The results for $\langle\Delta v\rangle$ are cross-checked with results from the autocorrelation method (Mosser \& Appourchaux 2009).

- Huber et al. (2009, hereafter SYD) compute $v_{\max }$ as the peak of the smoothed power spectrum. The smoothed power spectrum is computed using a Gaussian with a $F W H M$ of $2\langle\Delta v\rangle$. $\langle\Delta v\rangle$ is computed from the autocorrelation of the power spectrum in the frequency interval $v_{\max } \pm 5-7\langle\Delta v\rangle_{\exp }$, where $\langle\Delta v\rangle_{\exp }$ is calculated using Eq. (2).

- In the next method (Buzasi, Preston; hereafter DLB), $v_{\max }$ is determined from a Gaussian fit to the smoothed power spectrum. The power spectrum is smoothed using a Gaussian filter with a width equal to $10 \mu \mathrm{Hz}$. $\langle\Delta v\rangle$ is computed from the autocorrelation of the power spectrum in the range $v_{\max } \pm$ $v_{\max } / 4$.

- In the method described by Hekker et al. (2010a) and adapted for red giants, $v_{\max }$ is determined in two ways. In the first method (OCT I), $v_{\max }$ is defined as the centroid of a Gaussian fit to the smoothed power spectrum. In the second method (OCT II), $v_{\max }$ is computed as the first moment of the area under the smoothed power envelope. The smoothing is obtained using a boxcar with a width of $2\langle\Delta v\rangle .\langle\Delta v\rangle$ is computed from the $\mathrm{PS} \otimes \mathrm{PS}$ of the full frequency range in which oscillation excess has been detected.

From this overview it is clear that similar approaches are used by the different teams to determine $v_{\max }$ and $\langle\Delta v\rangle$, although there are differences in the smoothing for $v_{\max }$ and in the range over which the mean $\Delta v$ is computed. On average, neither of these effects are significant in comparison with other sources of uncertainty. We illustrate this for $\langle\Delta v\rangle$ with the data shown in Fig. 1, which shows the results from several methods for $v_{\max }$ and $\langle\Delta v\rangle$ for five typical stars. The observed values are shown by the symbols with the ranges over which $\langle\Delta v\rangle$ has been computed indicated by the small horizontal lines. Formal errors, where available, are also shown. It is obvious from this figure that the impact of the frequency range is small and within the uncertainties. This is plausible for the following reasons. Firstly, the trend in $\Delta v$ over a typical frequency range is approximately linear with frequency. Note that, when analysed in detail, it can be shown that $\Delta v$ may vary rapidly with frequency (Mosser et al. 2010). Secondly, most of the time, the region selected is symmetrical with respect to $v_{\max }$. The consequence of these two factors is that the value returned for $\langle\Delta v\rangle$ is roughly independent of the precise range chosen. Furthermore, $\Delta v$ changes relatively slowly with frequency and so the impact of a small change in the central position is small, i.e., within the general uncertainties in the determination of $\langle\Delta v\rangle$.

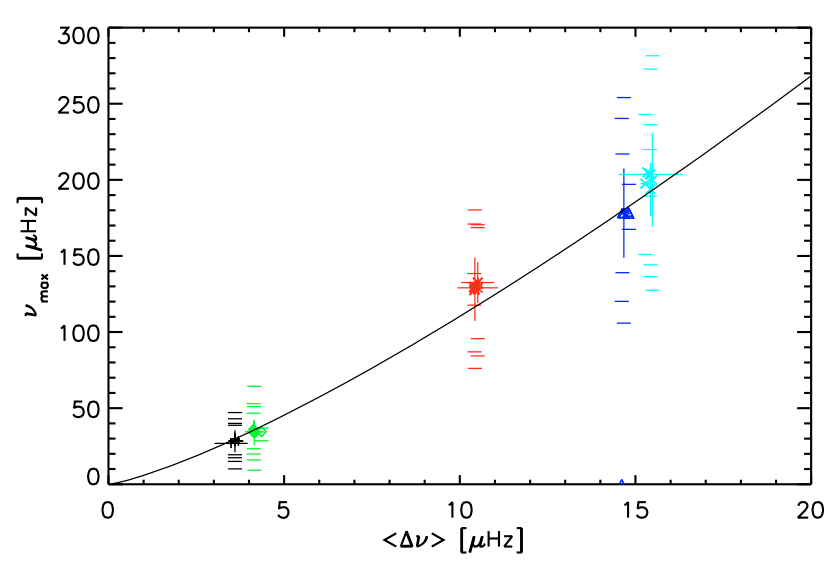

Fig. 1. $v_{\max }$ versus $\langle\Delta v\rangle$ for five stars observed with Kepler. For each star all results are plotted with the uncertainties derived by the different methods. The ranges in frequency over which $\langle\Delta v\rangle$ values are computed are indicated with the horizontal lines above and below the results. A different colour and symbol are used for results of each star. The black solid line indicates the approximate relation between $\langle\Delta v\rangle$ and $v_{\max }$.

Differences in results for $v_{\max }$ and $\langle\Delta v\rangle$ may also arise from the following issues:

- the preparation of the power spectrum;

- identification of the frequency range of oscillations;

- computation of the background signal;

- definition of a positive detection / artefact;

- computation of uncertainties.

These issues are addressed in Sects. 4 and 5.

\section{Comparison using simulations}

To obtain a measure of the variation in the observed parameters due to the stochastic nature of the oscillations, which we will refer to as realization noise, we first present results of a comparison using different realizations of simulated timeseries. Because we observe stochastically excited oscillations, the observed oscillation characteristics will change with time. Timeseries with an infinite timespan would provide the "real" or "limit" oscillation parameters. In reality we are dealing with data with a limited timespan and to investigate the scatter in the results due to this, we simulated 100 realizations for five "Kepler" stars using the simulator described by De Ridder et al. (2006). The input parameters are listed in Table 2 and were derived from the observations of the Kepler targets with Kepler ID 1720425, 2013502, 
Table 2. Input parameters for synthetic timeseries.

\begin{tabular}{cccccccccc}
\hline \hline Simulation & $\begin{array}{c}v_{\max } \\
\mu \mathrm{Hz}\end{array}$ & $\begin{array}{c}\Delta v \\
\mu \mathrm{Hz}\end{array}$ & $\begin{array}{c}\delta v_{02} \\
\mu \mathrm{Hz}\end{array}$ & $\begin{array}{c}\text { width }_{\text {env }} \\
\mu \mathrm{Hz}\end{array}$ & $\begin{array}{c}\text { height } \\
10^{3} \mathrm{ppm}^{2} \mu \mathrm{Hz}^{-1}\end{array}$ & $\begin{array}{c}\tau_{\ell=0} \\
\text { days }\end{array}$ & $\begin{array}{c}\tau_{\ell=1} \\
\text { days }\end{array}$ & $\begin{array}{c}\tau_{\ell=2} \\
\text { days }\end{array}$ & $\begin{array}{c}\tau_{\ell=3} \\
\text { days }\end{array}$ \\
\hline 1 & 20.0 & 2.7 & 0.38 & 5.0 & 40.0 & 50.0 & 15.0 & 30.0 & 30.0 \\
2 & 50.0 & 6.5 & 0.80 & 6.5 & 10.0 & 50.0 & 15.0 & 30.0 & 30.0 \\
3 & 80.0 & 9.1 & 1.2 & 6.5 & 14.4 & 50.0 & 15.0 & 30.0 & 30.0 \\
4 & 120.0 & 11.2 & 1.4 & 5.0 & 19.6 & 50.0 & 15.0 & 30.0 & 30.0 \\
5 & 170.0 & 13.9 & 1.7 & 7.0 & 6.4 & 50.0 & 15.0 & 30.0 & 30.0 \\
6 & 20.0 & 2.7 & 0.38 & 5.0 & 40.0 & 100.0 & 30.0 & 60.0 & 60.0 \\
7 & 50.0 & 6.5 & 0.80 & 6.5 & 10.0 & 100.0 & 30.0 & 60.0 & 60.0 \\
8 & 80.0 & 9.1 & 1.2 & 6.5 & 14.4 & 100.0 & 30.0 & 60.0 & 60.0 \\
9 & 120.0 & 11.2 & 1.4 & 5.0 & 19.6 & 100.0 & 30.0 & 60.0 & 60.0 \\
10 & 170.0 & 13.9 & 1.7 & 7.0 & 6.4 & 100.0 & 30.0 & 60.0 & 60.0 \\
\hline
\end{tabular}

Notes. Frequency of maximum oscillation power $\left(v_{\max }\right)$, large separation between modes of consecutive order $(\Delta v)$, small separation between modes with degree 0 and $2\left(\delta v_{02}\right)$, half width at half maximum of a Gaussian fit to the oscillation power excess (width env $_{\text {) }}$ in $\mu \mathrm{Hz}$, the height of this Gaussian fit (height ${ }_{\text {env }}$ ) to the power excess envelope in $\mathrm{ppm}^{2} \mu \mathrm{Hz}^{-1}$ and mode life times $(\tau)$ for modes of different degree $\ell=(0,1,2,3)$ in days.
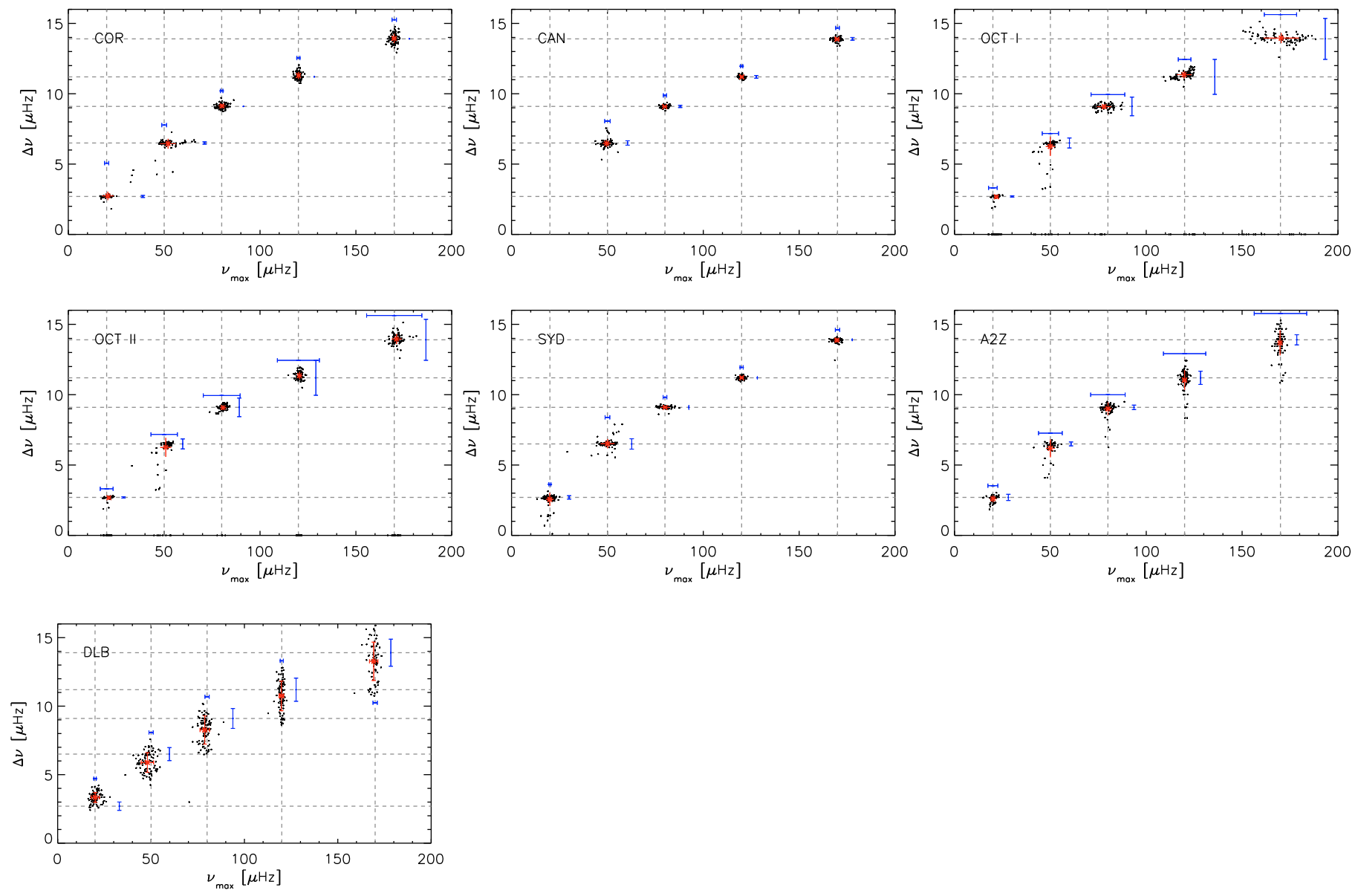

Fig. 2. Results for $v_{\max }$ and $\Delta v$ of the analysis of synthetic time series using different methods: COR, CAN, OCT I, OCT II, SYD, A2Z and DLB. The black dots indicate the values obtained by the methods for each individual realization, the red asterisks indicate the mean value and scatter of these results. The typical uncertainties on the computed values for each model are indicated with the blue error bars. The gray dashed lines indicate the input values.

2696732, 3526061, and 6033938 (see Table 7 for their characteristics). These stars are selected based on a visual inspection of their power spectra. By taking parameters obtained from observed stars instead of from for instance scaling relations, we aim to resemble the Kepler observations as well as possible. This also means that scaling relations are not readily applicable anymore, because stars with for instance different metallicity and/or mass are likely to be represented. These parameters are known to induce deviations from the scaling relations. As indicated in Table 2, two sets of mode life times have been considered for each of the simulated Kepler stars. This was done to investigate the influence of the width of the frequency peaks, 
S. Hekker et al.: Comparison of global oscillation parameters from different methods
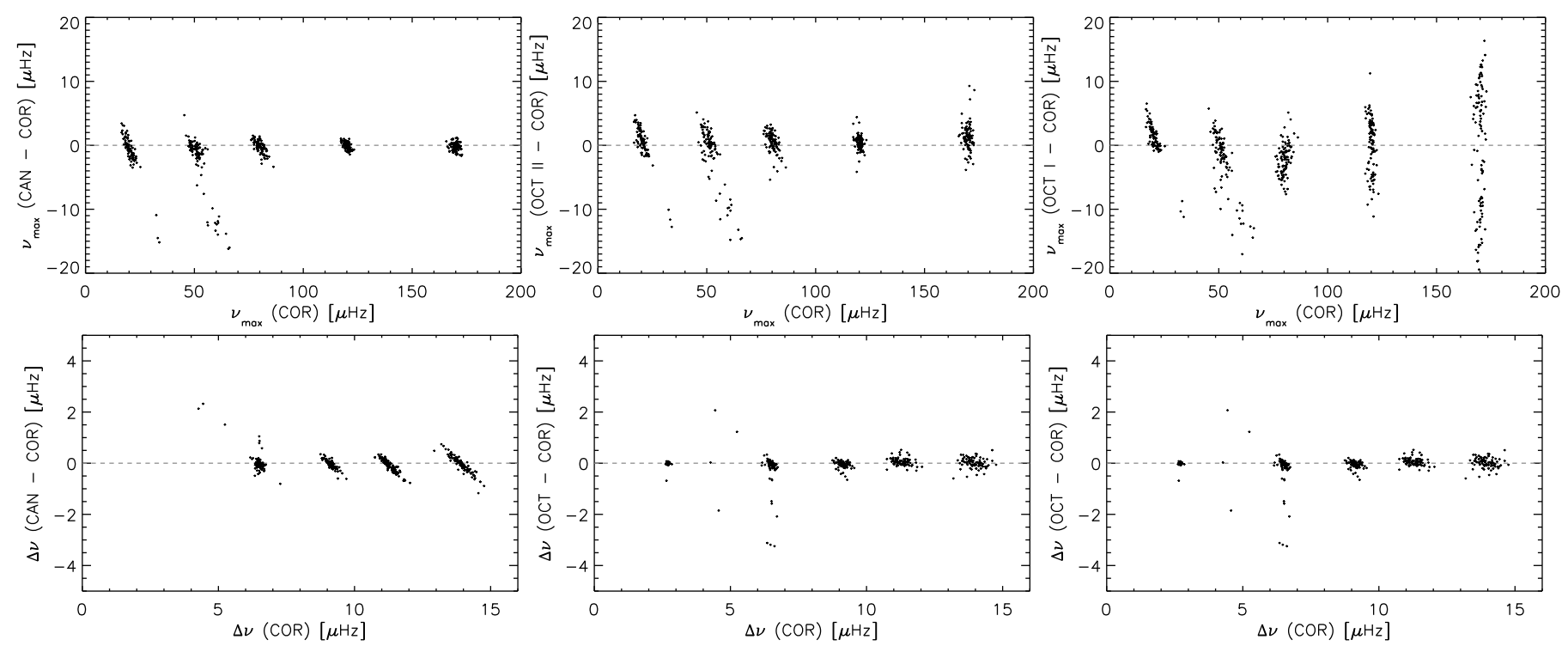

Fig. 3. The differences in the simulation results between different methods, from left to right CAN-COR, OCT II-COR, OCT I-COR are shown as a function of the value returned by COR. The top row is for $v_{\max }$ and the bottom row for $\Delta v$. The gray dashed line indicates agreement between the methods.
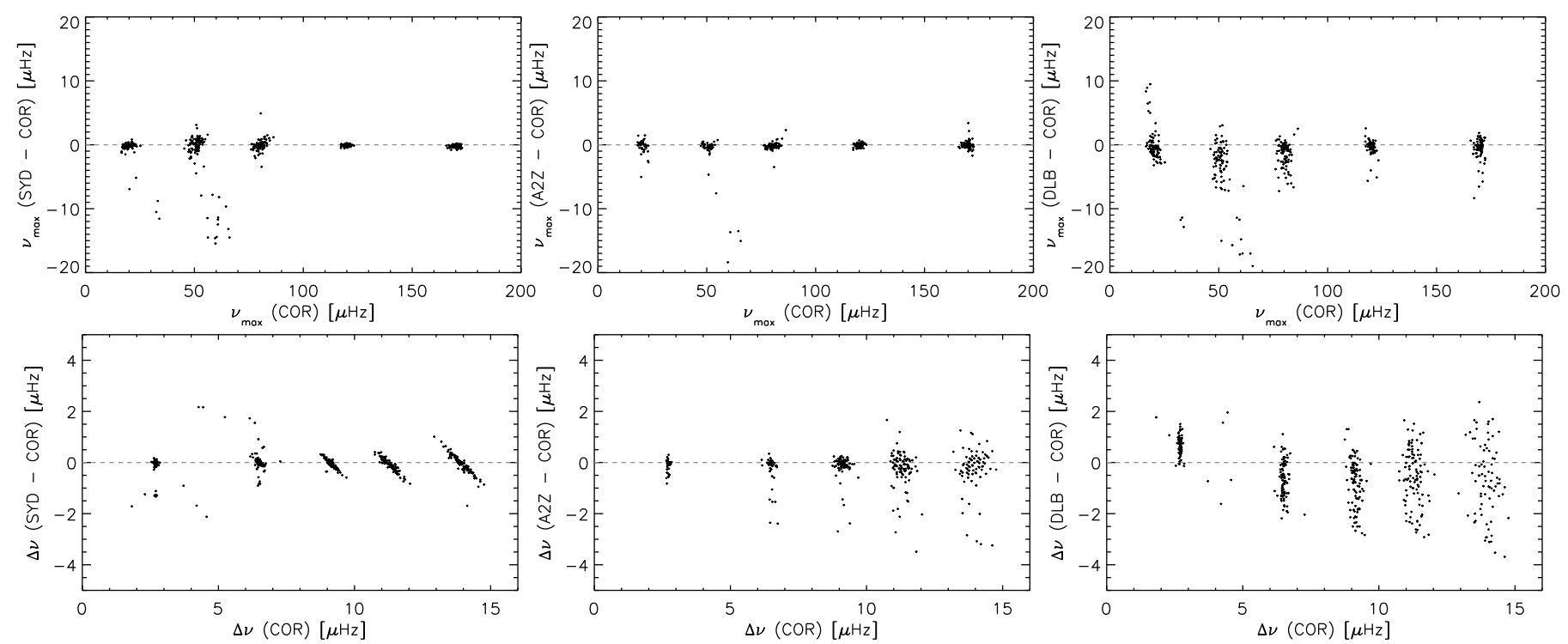

Fig. 4. Same as Fig. 3 but now from left to right for SYD-COR, A2Z-COR and, DLB-COR.

which increases for modes with decreasing life times, on the resulting values of $v_{\max }$ and $\langle\Delta v\rangle$.

These synthetic timeseries were analysed using the methods described in the previous section. The results for simulations 1-5 are shown in Fig. 2 and compared in Figs. 3-5, in which the comparison is shown as a function of the results obtained with method COR. Apart from results for some individual realizations around $v_{\max }$ of $50 \mu \mathrm{Hz}$, for which the oscillations have the smallest height compared to the background, the results are consistent with the input values, but with a non-negligible scatter. This scatter is inherent to the observations of the star, although not fully independent of the analysis method, and should be taken into account when detailed modelling is performed for a particular star. The mean values of the results of all realizations with the same input parameters and the standard deviations of the results are listed in Tables 3 and 4. The results for simulations 6-10 with longer mode life times are very similar to the results obtained for simulations $1-5$. Therefore, we conclude that the mode life time does not significantly influence the obtained $v_{\max }$ and $\langle\Delta v\rangle$ at least for the values investigated here.

Figures 2-4 show some interesting differences in the strengths and weaknesses of the different methods. Firstly, from Fig. 2 and Tables 3 and 4 it is clear that all methods provide in general results consistent with the input parameters. Only the Lorentzian fitting from CAN did not return values for $\langle\Delta v\rangle$ for the simulations with $\nu_{\max }$ at $20 \mu \mathrm{Hz}$, because the frequency peaks were spaced too closely relative to the timebase of the dataset. In general, for the realizations at $\nu_{\max }$ of $50 \mu \mathrm{Hz}$ and $170 \mu \mathrm{Hz}$ the spread in the results is larger due to the relative weakness of the oscillations, and the results of some methods show more spread in $v_{\max }$ (OCT I), while for others the spread in $\langle\Delta v\rangle$ is somewhat larger (A2Z and DLB). Secondly, when comparing the results of the different methods with respect to COR (see Figs. 3 and 4) this shows that, apart from the "outliers", the 

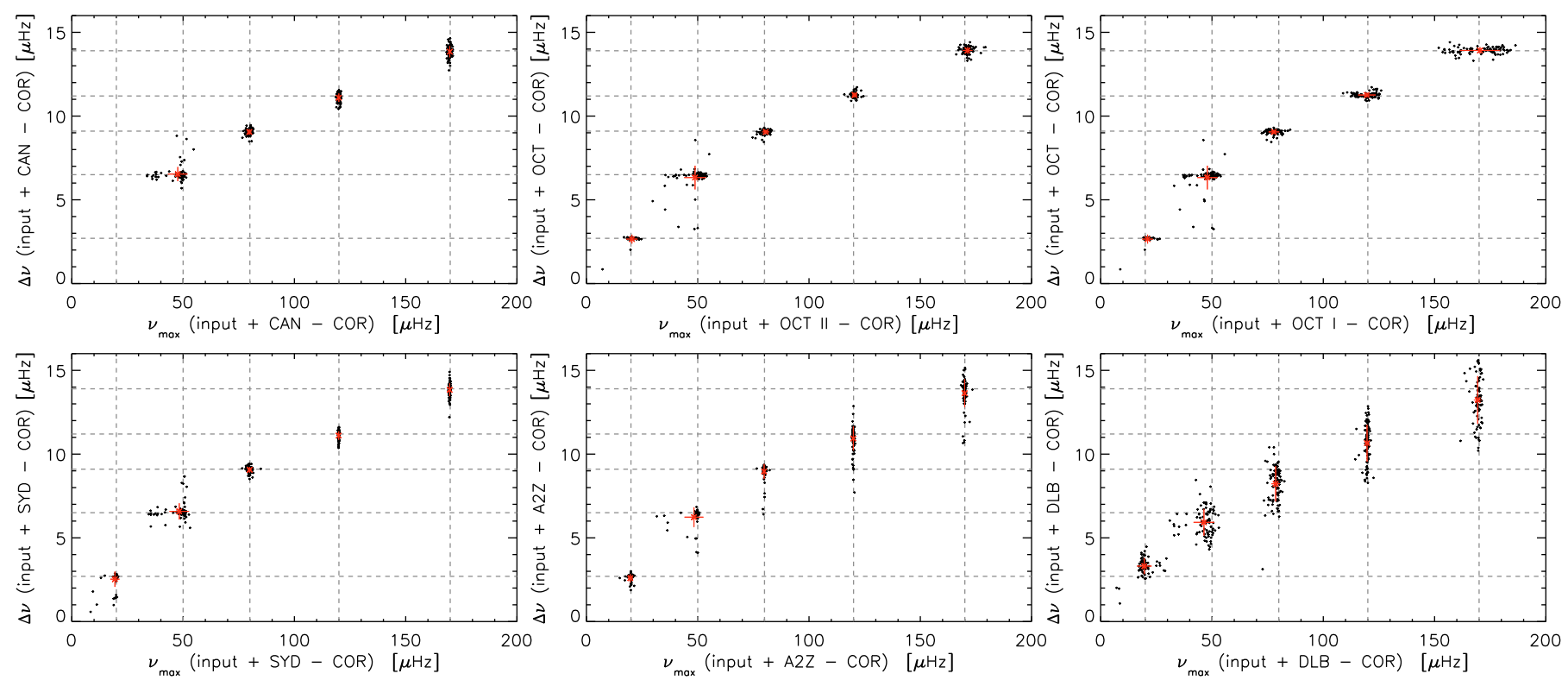

Fig. 5. Same as Fig. 2, but here we show the difference in the results from different methods, i.e., from left to right and top to bottom CAN-COR, OCT II-COR, OCT I-COR, SYD-COR, A2Z-COR and, DLB-COR, at the position of the input parameters of the simulation. The mean values and the scatter are indicated with the red asterisks.

Table 3. Mean and standard deviations of the computed $v_{\max }$ for synthetic timeseries.

\begin{tabular}{|c|c|c|c|c|c|c|c|c|}
\hline $\operatorname{sim}$ & $\begin{array}{l}v_{\max } \\
\text { input } \\
\mu \mathrm{Hz}\end{array}$ & $\begin{array}{l}v_{\max } \\
\mathrm{CAN} \\
\mu \mathrm{Hz}\end{array}$ & $\begin{array}{l}v_{\max } \\
\mathrm{COR} \\
\mu \mathrm{Hz}\end{array}$ & $\begin{array}{c}v_{\max } \\
\text { OCT I } \\
\mu \mathrm{Hz}\end{array}$ & $\begin{array}{c}v_{\max } \\
\text { OCT II } \\
\mu \mathrm{Hz}\end{array}$ & $\begin{array}{l}v_{\max } \\
\mathrm{SYD} \\
\mu \mathrm{Hz}\end{array}$ & $\begin{array}{l}v_{\max } \\
\mathrm{A} 2 \mathrm{Z} \\
\mu \mathrm{Hz}\end{array}$ & $\begin{array}{l}v_{\max } \\
\mathrm{DLB} \\
\mu \mathrm{Hz}\end{array}$ \\
\hline 1 & 20.0 & $19.6 \pm 1.0$ & $20.4 \pm 2.3$ & $21.8 \pm 1.2$ & $21.2 \pm 1.2$ & $19.9 \pm 2.0$ & $20.1 \pm 1.8$ & $20.1 \pm 2.1$ \\
\hline 6 & 20.0 & $19.4 \pm 1.1$ & $20.3 \pm 2.3$ & $21.9 \pm 1.2$ & $21.1 \pm 1.7$ & $19.5 \pm 2.4$ & $20.6 \pm 1.9$ & $20.3 \pm 2.3$ \\
\hline 2 & 50.0 & $49.6 \pm 2.0$ & $52.1 \pm 4.0$ & $50.0 \pm 2.3$ & $50.8 \pm 2.9$ & $50.1 \pm 3.6$ & $50.0 \pm 2.3$ & $48.0 \pm 3.3$ \\
\hline 7 & 50.0 & $49.6 \pm 2.3$ & $52.9 \pm 4.4$ & $50.0 \pm 2.7$ & $51.1 \pm 2.6$ & $50.1 \pm 3.6$ & $49.5 \pm 3.3$ & $47.6 \pm 4.8$ \\
\hline 3 & 80.0 & $79.9 \pm 1.4$ & $80.2 \pm 1.9$ & $78.0 \pm 3.6$ & $80.8 \pm 1.7$ & $80.1 \pm 2.3$ & $80.1 \pm 2.1$ & $78.8 \pm 2.5$ \\
\hline 8 & 80.0 & $79.8 \pm 1.4$ & $79.9 \pm 1.8$ & $77.9 \pm 3.5$ & $80.7 \pm 1.5$ & $79.8 \pm 2.3$ & $79.8 \pm 1.8$ & $78.7 \pm 2.2$ \\
\hline 4 & 120.0 & $120.1 \pm 1.0$ & $120.1 \pm 1.1$ & $119.8 \pm 3.8$ & $120.6 \pm 1.4$ & $120.0 \pm 1.1$ & $120.0 \pm 1.2$ & $119.8 \pm 1.3$ \\
\hline 9 & 120.0 & $119.8 \pm 1.0$ & $119.8 \pm 1.2$ & $118.9 \pm 4.1$ & $120.4 \pm 1.4$ & $119.7 \pm 1.3$ & $119.7 \pm 1.3$ & $119.4 \pm 1.6$ \\
\hline 5 & 170.0 & $169.8 \pm 1.5$ & $170.0 \pm 1.4$ & $170.4 \pm 9.2$ & $171.2 \pm 2.6$ & $169.7 \pm 1.4$ & $169.8 \pm 0.9$ & $169.2 \pm 2.2$ \\
\hline 10 & 170.0 & $169.7 \pm 1.5$ & $169.9 \pm 1.8$ & $169.2 \pm 9.9$ & $170.8 \pm 3.4$ & $169.7 \pm 1.9$ & $169.8 \pm 0.9$ & $168.5 \pm 2.9$ \\
\hline
\end{tabular}

Table 4. Mean and standard deviations of the computed $\langle\Delta v\rangle$ for synthetic timeseries.

\begin{tabular}{|c|c|c|c|c|c|c|c|}
\hline sim & $\begin{array}{c}\Delta v \\
\text { input } \\
\mu \mathrm{Hz}\end{array}$ & $\begin{array}{l}\Delta v \\
\text { CAN } \\
\mu \mathrm{Hz}\end{array}$ & $\begin{array}{c}\Delta v \\
\mathrm{COR} \\
\mu \mathrm{Hz}\end{array}$ & $\begin{array}{c}\Delta v \\
\mathrm{OCT} \\
\mu \mathrm{Hz}\end{array}$ & $\begin{array}{c}\Delta v \\
\text { SYD } \\
\mu \mathrm{Hz}\end{array}$ & $\begin{array}{c}\Delta v \\
\mathrm{~A} 2 \mathrm{Z} \\
\mu \mathrm{Hz}\end{array}$ & $\begin{array}{c}\Delta v \\
\mathrm{DLB} \\
\mu \mathrm{Hz}\end{array}$ \\
\hline 1 & 2.7 & & $2.7 \pm 0.2$ & $2.7 \pm 0.2$ & $2.6 \pm 0.5$ & $2.6 \pm 0.2$ & $3.3 \pm 0.4$ \\
\hline 6 & 2.7 & & $2.70 \pm 0.04$ & $2.7 \pm 0.2$ & $2.7 \pm 0.2$ & $2.7 \pm 0.2$ & $3.4 \pm 1.3$ \\
\hline 2 & 6.5 & $6.5 \pm 0.3$ & $6.4 \pm 0.3$ & $6.3 \pm 0.7$ & $6.5 \pm 0.3$ & $6.2 \pm 0.6$ & $5.9 \pm 0.7$ \\
\hline 7 & 6.5 & $6.5 \pm 0.2$ & $6.5 \pm 0.1$ & $6.2 \pm 0.7$ & $6.5 \pm 0.3$ & $6.0 \pm 0.8$ & $6.1 \pm 0.9$ \\
\hline 3 & 9.1 & $9.1 \pm 0.1$ & $9.1 \pm 0.2$ & $9.1 \pm 0.2$ & $9.1 \pm 0.1$ & $9.0 \pm 0.5$ & $8.3 \pm 1.0$ \\
\hline 8 & 9.1 & $9.1 \pm 0.1$ & $9.1 \pm 0.2$ & $9.1 \pm 0.2$ & $9.1 \pm 0.1$ & $9.0 \pm 0.6$ & $8.4 \pm 0.9$ \\
\hline 4 & 11.2 & $11.2 \pm 0.1$ & $11.3 \pm 0.3$ & $11.4 \pm 0.3$ & $11.2 \pm 0.1$ & $11.0 \pm 0.7$ & $10.7 \pm 1.1$ \\
\hline 9 & 11.2 & $11.2 \pm 0.1$ & $11.3 \pm 0.3$ & $11.4 \pm 0.3$ & $11.2 \pm 0.1$ & $11.0 \pm 0.8$ & $10.9 \pm 1.1$ \\
\hline 5 & 13.9 & $13.9 \pm 0.1$ & $13.9 \pm 0.3$ & $14.0 \pm 0.4$ & $13.9 \pm 0.2$ & $13.7 \pm 0.9$ & $13.3 \pm 1.4$ \\
\hline 10 & 13.9 & $13.9 \pm 0.1$ & $14.0 \pm 0.3$ & $14.1 \pm 0.3$ & $13.9 \pm 0.1$ & $13.8 \pm 0.9$ & $13.7 \pm 1.5$ \\
\hline
\end{tabular}

results for $\Delta v$ of the different methods agree to sub- $\mu \mathrm{Hz}$ level. The agreement in $\nu_{\max }$ is typically a few $\mu \mathrm{Hz}$. Larger differences are only present for oscillations at the highest frequency when using the OCT I method (centroid of Gaussian fit to smoothed power excess). From the trends in the centre top panel in Fig. 3 it is clear that for $v_{\max }$ the spread in the results at low frequencies is largest for results obtained with COR, i.e., the spread of the results on the $x$-axis (COR) is equivalent to the spread on the $y$-axis (OCT II-COR). At higher frequencies the differences in $v_{\max }$ seem to be mainly due to OCT II. In this case the spread in results on the $x$-axis (COR) is less than the spread on the $y$-axis (OCTÍI-COR). Similar reasoning reveals that for $\Delta v$ at $v_{\max }>80 \mu \mathrm{Hz}$ the spread in the results obtained with method COR dominate the total spread in the difference between the results CAN-COR (left bottom panel in Fig. 3) and SYD-COR (left bottom panel in Fig. 4). 

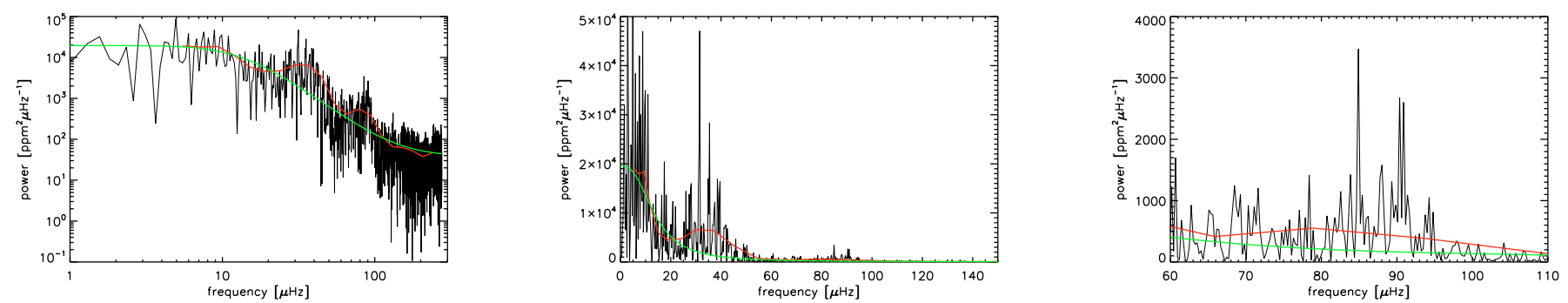

Fig. 6. Power spectra of a target (KIC4813971) on CCD 2 showing power excess due to solar-like oscillations at frequencies around $35 \mu \mathrm{Hz}$ and the artefact due to desaturation around $87 \mu \mathrm{Hz}$. The red line indicates a binned power spectrum and the green line a fit to the background.

Table 5. The standard deviations of the differences in results for $v_{\max }$ from different methods.

\begin{tabular}{cccccccc}
\hline \hline $\operatorname{sim}$ & $\begin{array}{c}v_{\max } \\
\text { input } \\
\mu \mathrm{Hz}\end{array}$ & $\begin{array}{c}\sigma_{v_{\max }} \\
\text { CAN-COR } \\
\mu \mathrm{Hz}\end{array}$ & $\begin{array}{c}\sigma_{v_{\max }} \\
\text { OCT I-COR } \\
\mu \mathrm{Hz}\end{array}$ & $\begin{array}{c}\sigma_{v_{\max }} \\
\text { OCT II-COR } \\
\mu \mathrm{Hz}\end{array}$ & $\begin{array}{c}\sigma_{v_{\max }} \\
\text { SYD-COR } \\
\mu \mathrm{Hz}\end{array}$ & $\begin{array}{c}\sigma_{v_{\max }} \\
\text { A2Z-COR } \\
\mu \mathrm{Hz}\end{array}$ & $\begin{array}{c}\sigma_{v_{\max }} \\
\text { DLB-COR } \\
\mu \mathrm{Hz}\end{array}$ \\
\hline 1 & 20.0 & 2.9 & 2.4 & 2.5 & 2.1 & 1.1 & 3.3 \\
6 & 20.0 & 2.3 & 2.5 & 2.7 & 2.5 & 2.1 & 2.7 \\
2 & 50.0 & 4.4 & 4.5 & 4.9 & 4.4 & 4.2 & 4.7 \\
7 & 50.0 & 5.4 & 5.0 & 5.3 & 6.2 & 5.5 & 6.7 \\
3 & 80.0 & 0.9 & 2.4 & 1.6 & 0.9 & 0.5 & 1.9 \\
8 & 80.0 & 0.9 & 2.6 & 1.2 & 0.8 & 0.4 & 1.6 \\
4 & 120.0 & 0.6 & 3.9 & 1.1 & 0.2 & 0.3 & 1.0 \\
9 & 120.0 & 0.7 & 4.2 & 1.3 & 0.8 & 0.3 & 1.2 \\
5 & 170.0 & 0.6 & 9.0 & 2.1 & 0.2 & 0.6 & 1.7 \\
10 & 170.0 & 0.7 & 9.3 & 2.9 & 0.3 & 0.4 & 1.5 \\
\hline
\end{tabular}

The differences in the results can be due to differences in the methods and due to the realization noise. To investigate this we show the differences in the results added to the input value (see Fig. 5). The scatter in the values in this figure can be interpreted as the differential response of the methods, and are listed in Tables 5 and 6. From this table it is notable that for $\langle\Delta v\rangle$ of oscillations with $v_{\max }$ at $80 \mu \mathrm{Hz}$ or above, the scatter between OCT and COR is less than the scatter in Fig. 2. This indicates that in these cases the scatter due to realization noise is at least as important as the scatter in the results due to the different methods. We also see that the scatter in the difference of the results from SYD-COR and A2Z-COR for $v_{\max }$ is less for $\nu_{\max } \geq 80 \mu \mathrm{Hz}$. For the other simulations we cannot distinguish between realization noise and uncertainties, possibly due to the effect of beating between signal and background noise, i.e. changes in the amplitude and hence power when the signal and noise are of similar magnitudes due to the fact that in the amplitude spectrum the signal and noise are added as complex numbers and their relative phases can change significantly. These results indicate that the spread in results due to realization noise can be larger than the difference in the results between different methods, but this depends on the frequency range and the methods used.

Finally, the error bars in Fig. 2 show that uncertainties on the results are in general not a reliable indication of the spread in the results. We discuss the issue of the uncertainties further in Sect. 5.

\section{Observations}

For the present investigation we used data obtained by the NASA Kepler satellite taken during the 10 day commissioning run (Q0), the one-month first roll (Q1) and the three-month second roll (Q2), all taken in long-cadence mode (29.4-min sampling, not entirely regular due to changes in the correction to barycentric
Table 6. The standard deviations of the differences in results for $\langle\Delta v\rangle$ from different methods.

\begin{tabular}{|c|c|c|c|c|c|c|}
\hline $\operatorname{sim}$ & $\begin{array}{c}\Delta v \\
\text { input } \\
\mu \mathrm{Hz}\end{array}$ & $\begin{array}{c}\sigma_{\Delta v} \\
\mathrm{CAN}-\mathrm{COR} \\
\mu \mathrm{Hz}\end{array}$ & $\begin{array}{c}\sigma_{\Delta v} \\
\text { OCT-COR } \\
\mu \mathrm{Hz}\end{array}$ & $\begin{array}{c}\sigma_{\Delta v} \\
\text { SYD-COR } \\
\mu \mathrm{Hz}\end{array}$ & $\begin{array}{c}\sigma_{\Delta v} \\
\mathrm{~A} 2 \mathrm{Z}-\mathrm{COR} \\
\mu \mathrm{Hz}\end{array}$ & $\begin{array}{c}\sigma_{\Delta v} \\
\text { DLB-COR } \\
\mu \mathrm{Hz}\end{array}$ \\
\hline 1 & 2.7 & & 0.3 & 0.5 & 0.2 & 0.5 \\
\hline 6 & 2.7 & & 0.2 & 0.2 & 0.2 & 1.3 \\
\hline 2 & 6.5 & 0.4 & 0.7 & 0.5 & 0.6 & 0.8 \\
\hline 7 & 6.5 & 0.2 & 0.7 & 0.3 & 0.8 & 1.0 \\
\hline 3 & 9.1 & 0.2 & 0.1 & 0.2 & 0.4 & 1.1 \\
\hline 8 & 9.1 & 0.2 & 0.1 & 0.2 & 0.6 & 0.9 \\
\hline 4 & 11.2 & 0.3 & 0.1 & 0.3 & 0.7 & 1.1 \\
\hline 9 & 11.2 & 0.3 & 0.2 & 0.3 & 0.8 & 1.1 \\
\hline 5 & 13.9 & 0.3 & 0.2 & 0.4 & 0.8 & 1.4 \\
\hline 10 & 13.9 & 0.3 & 0.3 & 0.3 & 0.8 & 1.5 \\
\hline
\end{tabular}

times caused by the satelite orbit, see for more details Jenkins et al. 2010). These data are of unprecedented quality, although there are still some outliers and artefacts present in the timeseries of some stars. Before the power spectra are computed, outliers present in the timeseries need to be treated. Their positions can either be filled with zeroes or with a mean flux computed from surrounding observations, or left blank. In Q2 there was a safemode event in the first month during which the satellite warmed up and the subsequent thermal relaxation produced photometric responses. In addition there were three separate adjustments of the pointing at the $\sim 0.05$ pixel level, leading to step functions in the photometry in some cases. We investigated several ways to remove these signals, such as removing the affected part of the timeseries, or splitting the light curve into several parts, and correcting them individually. These different methods seem to work equally well and therefore we do not expect significant differences in the results of the oscillation parameters described here due to these corrections. 

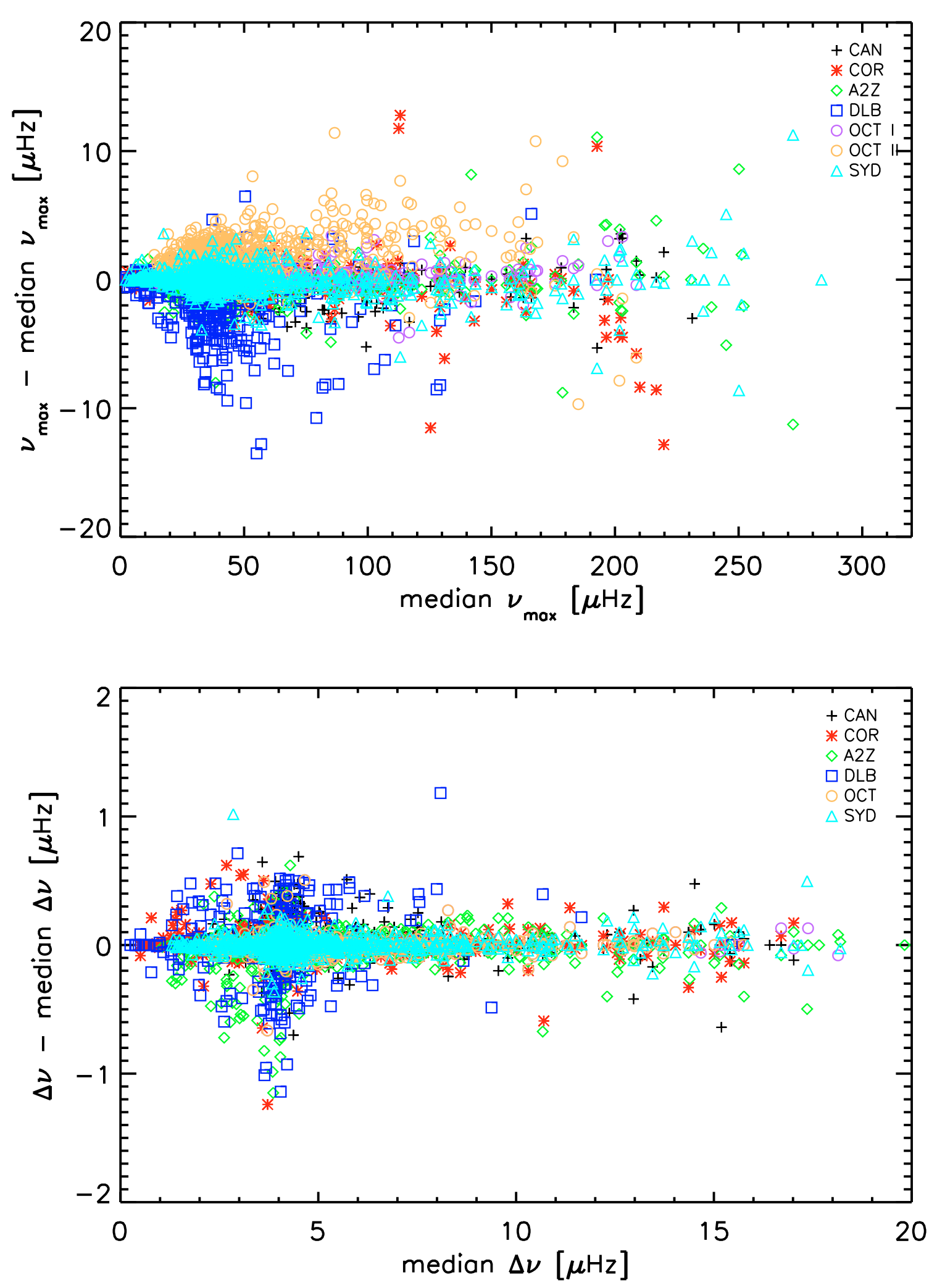

Fig. 7. Results of all methods represented as the value-median value versus the median value for $v_{\max }$ (top) and $\langle\Delta v\rangle($ bottom). The different colours and symbols represent results from the different methods, as indicated in the legend.

Investigation of the data taken during the first roll (Q1) revealed that many science targets exhibit non-sinusoidal oscillations with a $3.2 \mathrm{~h}$ period $(87 \mu \mathrm{Hz})$. This phenomenon is associated with cycling of a heater on one of the Kepler reaction wheels. For an example of this signature see Fig. 6. Due to the non-sinusoidal nature of the signal the resulting signature in the power spectrum mimics solar-like oscillations in terms of its width and near equidistant features. We investigated whether 
it is related to CCD module or position on the CCD module. We found that it seems to be worse for CCD modules located on the edge of the complete 42-CCD mozaic and mostly on one side, i.e. CCDs 2, 3, 4, 6, 10, 11. So far this was only investigated for stars observed during the Q0 and Q1 runs.

\section{Comparison}

Comparing results from seven different methods that produced seven measures for $\left\langle v_{\max }\right\rangle$ and six measures for $\langle\Delta v\rangle$ for hundreds of stars is a lengthy process. The results of all teams are collected in a data exchange facility called the Catbasket. The Catbasket is used to store results for individual stars with uncertainties and explanations on how the results were obtained, written in a specific format. The Catbasket is located and maintained in Birmingham and is accessible through a web interface. The concept of the Catbasket allows for a comparison of results, as is done in this study.

Most methods have an automated procedure to detect the oscillations. These are based on either the hump of excess power (OCT, DLB) or on the regular frequency pattern of the oscillations (SYD, COR, A2Z). For the CAN method, the stars are selected manually and tend to be those where the signal is strong. The exact implementation of the oscillation detection influences the sensitivity of the methods in any given frequency range. Therefore, the number of methods that return a result varies from star to star.

Because of the differences between different methods, we resist giving "mean" values for $v_{\max }$ or $\langle\Delta v\rangle$ for any of the Kepler stars. However, we do wish to take advantage of the multiple determinations of the parameters to detect and remove values that are discrepant. For this purpose, we compare for each star the results from the individual methods with a median value taken over all results for that star. For the detections of outliers we use different definitions for $v_{\max }$ and $\langle\Delta v\rangle$. For the outlier rejection for $v_{\max }$ an absolute threshold of $15 \mu \mathrm{Hz}$ and a relative threshold of $25 \%$ are used. This reflects the roughly constant scatter above $30 \mu \mathrm{Hz}$, but below that level it is more dependent on the median value of $v_{\max }$ (see top panel of Fig. 7). This is also justified by the results of the simulations (Sect. 3) where the spread observed in the returned values for $v_{\max }$ is roughly constant and is less than $5 \mu \mathrm{Hz}$. If outlier rejection was applied for $\nu_{\max }$ we also excluded the matching value for $\langle\Delta v\rangle$. For the remaining outliers in $\langle\Delta v\rangle$ we applied a rejection based on the median absolute deviation (MAD). We use 10.MAD unless this is less than $0.5 \mu \mathrm{Hz}$, in which case $0.5 \mu \mathrm{Hz}$ is used as the cut-off. This reflects the fact that, for some stars, $\langle\Delta v\rangle$ is poorly determined. Note that a minimum of three results is required before outliers can be identified.

\section{Results and discussion}

The results for $v_{\max }$ and $\langle\Delta v\rangle$ from each method are listed in Table 7 and shown in Fig. 7. Results have been obtained for 1301 stars. In Fig. 8 the number of results obtained per star is shown as a function of $v_{\max }$ (left), and the fractional distribution of the number of results (right). This histogram shows that for $69 \%$ of the stars we have five or more results, with a clear maximum at six results. For $\nu_{\max }$ between roughly 50 and $170 \mu \mathrm{Hz}$ there are only very few stars with less than five results, which indicates that the majority of the methods are most sensitive in this frequency interval and less sensitive for oscillations at lower $(<50 \mu \mathrm{Hz})$ and higher $(>170 \mu \mathrm{Hz})$ frequencies for which fewer methods obtain results.
We use the median to compare individual results and explore evidence for bias and possible trends of a method compared to the median value of all results. This information can be useful to see how different a method is from the others, but does not by definition tell whether individual methods provide wrong or right values. It is for instance clear that the $v_{\max }$ values from the first moment of the smoothed power excess (OCT II) are generally higher than results from other methods. Also the spread in these results is relatively large compared to other results. Interestingly, the results of the simulations from the first moment (OCT II) give results that are closer to the input values, i.e. are less spread, than the results from a Gaussian fit to the smoothed power excess (OCT I). It is, however, clear that for the results of the observations compared to the median, the OCT I results are more consistent with the majority of the results, among which the COR, SYD and A2Z results, which also gave results relatively close to the input values of the simulations. Therefore, with the current results of the comparison we can not discard or favour either of the methods OCT I or OCT II, although their results show slightly different behaviour compared to other methods in the simulations and observational results, respectively.

For $\langle\Delta v\rangle$ no significant trends and biases are present in the results from different methods, but the scatter in the results seems to be larger for $\langle\Delta v\rangle$ between roughly 3 and $5 \mu \mathrm{Hz}$ compared to other values. From population studies (e.g. Miglio et al. 2009) it is known that stars with $\nu_{\max }$ between 30 and $40 \mu \mathrm{Hz}$ and $\langle\Delta v\rangle$ between 3 and $5 \mu \mathrm{Hz}$ are mainly red-clump stars. Due to the fact that stars remain for a relatively long time in the $\mathrm{He}$ burning phase in the red-clump, these stars are most commonly observed (Hekker et al. 2009; Mosser et al. 2010). If we bin the $\langle\Delta v\rangle$ values in $1 \mu \mathrm{Hz}$ bins we can see that most values are indeed returned between 3 and $5 \mu \mathrm{Hz}$, but that the standard deviation of these values is similar to that in bins at other values of $\langle\Delta v\rangle$. So the larger spread in the results in the $\langle\Delta v\rangle$ interval $3-5 \mu \mathrm{Hz}$ could be an apparent effect caused by the increased number of results. The scatter in the $\Delta v-v_{\max }$ relation (Eq. (2)) using results from the different methods is investigated by Huber et al. (2010).

Notwithstanding the differences between the methods and the trends and biases of individual results with respect to the median, the results of the different methods agree within a few percent, as shown in Fig. 9. These figures show the distribution of the ratio of the deviation over the median value of $v_{\max }$ and $\Delta v$ respectively. The deviation and median are correlated values and a bias in a method most likely causes the skewness in the $v_{\max }$ distribution.

We also investigate the relation between the absolute deviation from the median and the formal errors, i.e., uncertainties derived within each method, for both $v_{\max }$ and $\langle\Delta v\rangle$. These relations show immediately that for most methods the formal error does not provide a realistic indication of the absolute deviation from the median. Again, this does not tell whether the formal errors are unrealistic, but indicates that for some methods the scatter around the median is considerably different from the computed formal error. Additionally, the formal errors do also not provide an indication of the spread due to realization noise.

\section{Conclusions}

We have performed a comparison of results from different methods for the frequency of maximum oscillation power and the mean large frequency separation for over one thousand red-giant stars observed during the first four months of Kepler observations. We investigated the differences and consistency between 

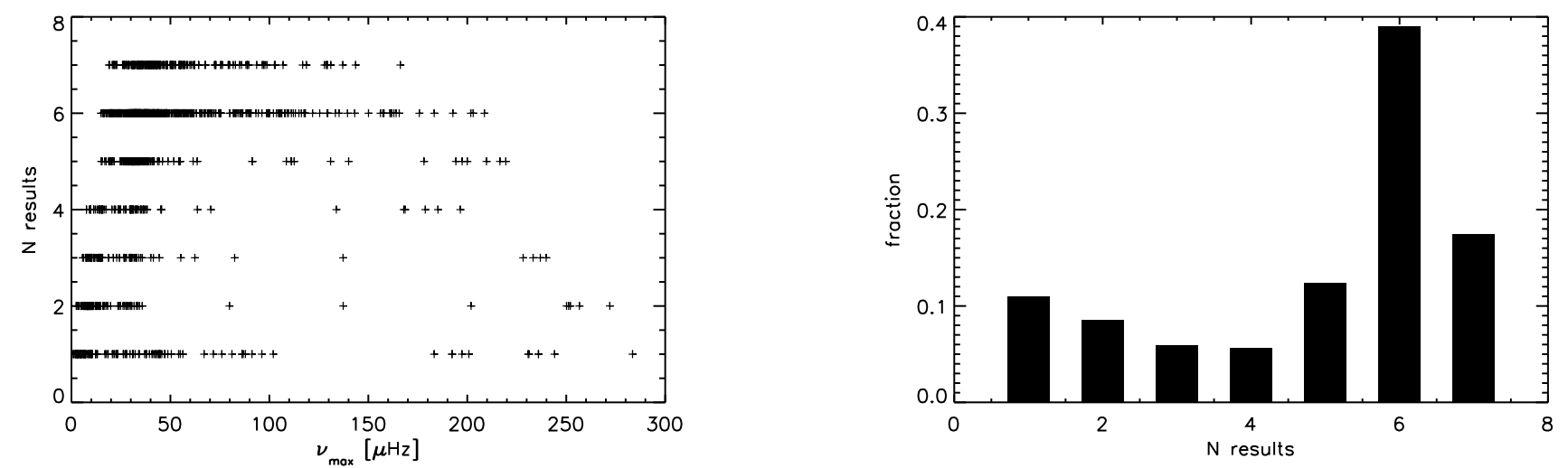

Fig. 8. Number of results obtained per star as a function of $v_{\max }(l e f t)$ and the fractional distribution of the number of results obtained per star (right).
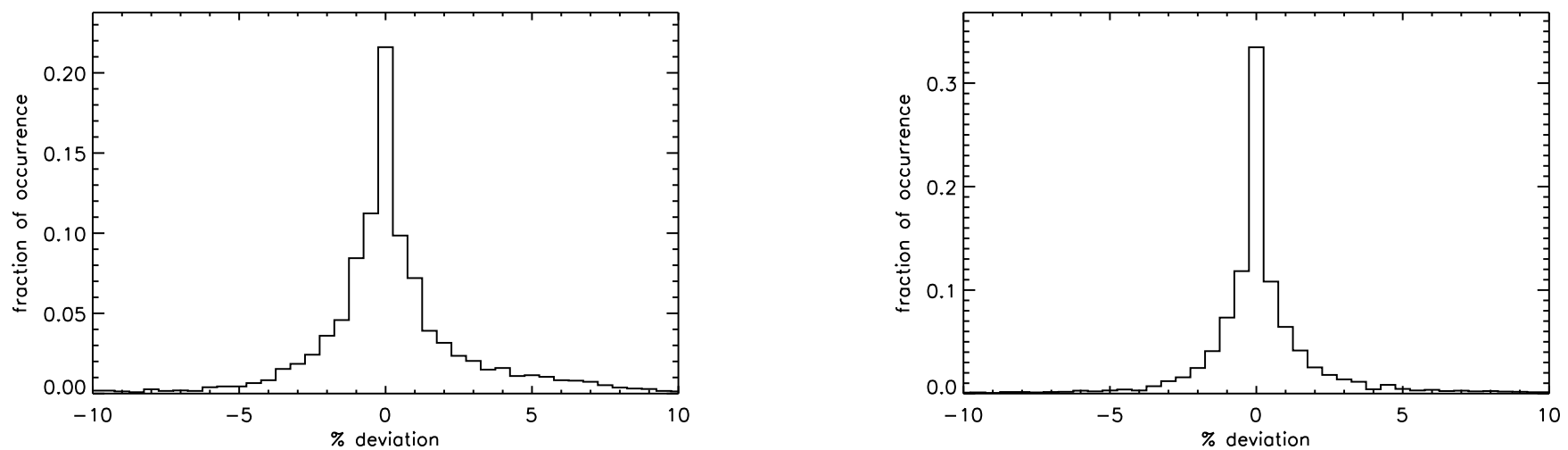

Fig. 9. Distributions of the ratio deviation/median value for $v_{\max }(l e f t)$ and $\langle\Delta v\rangle(r i g h t)$.

these results and their uncertainties. The effect of realization noise, originating from the stochastic nature of the oscillations, is also investigated using simulated datasets.

The comparison of different realizations of simulated data with the same input values revealed that the scatter due to realization noise is non-negligible and can be at least as important as the internal uncertainty of the result due to the method used, but this depends on the frequency of maximum oscillation power and on the methods, i.e., some methods are more sensitive to realization noise than others. When detailed modelling is performed for red-giant stars, the fact that the observational results are obtained from only one realization has to be taken into account.

For $69 \%$ of the 1301 stars with results, we could obtain $v_{\max }$ and $\langle\Delta v\rangle$ with five or more methods. For stars in a frequency range between 50 and $170 \mu \mathrm{Hz}$ this percentage is increased to $92 \%$ indicating that the majority of the methods are sensitive to oscillations in this frequency interval. At lower $(<50 \mu \mathrm{Hz})$ frequencies less results are obtained. This is firstly due to the limited frequency resolution of the data and secondly due to difficulties in disentangling oscillations from other effects, such as granulation or instrumental effects, which are also present at low frequencies. At higher $(>170 \mu \mathrm{Hz})$ frequencies the lower height, i.e. lower signal-to-noise ratio, of the oscillations causes more problems for some methods to detect the oscillations. Furthermore, the Nyquist frequency of these data is $\sim 280 \mu \mathrm{Hz}$, which possibly causes difficulties due to reflection effects, while for some stars only part of the oscillation power excess is covered. In these cases the determination of the background signal is also more complicated.

Although some biases and trends with respect to the median value of the results are present in each method, the results from the different methods agree for most stars within a few percent. Despite the consistency between the results from different methods, the stated formal errors are not indicative of the deviation of these results from the median value.

For $\langle\Delta v\rangle$, we found that the results do not show significant dependence on the range over which they were calculated. This is plausible because the trend in $\Delta v$ is approximately linear with frequency and/or shows rapid variations with relatively low amplitude (Mosser et al. 2010), which average out. This is the case for the red giants investigated here with oscillations over a range of roughly 10 to $280 \mu \mathrm{Hz}$.

Apart from the results described here, the comparison also helped to improve all the methods and will continue to do so. Although we are dealing with automated methods, it appeared that inspection of the results is important. In order to remove false positives, it remains necessary to inspect the results in specific frequency ranges by eye: at low frequencies, close to the Nyquist frequency or in regions of known artefacts as described in Sect. 4. An additional inspection has been performed for stars for which the results of different methods were significantly different. The latter inspection revealed in most cases that different features were selected as being the oscillations. In some cases this could be traced back to artefacts, as discussed in Sect. 4, or contamination of background stars. 
To summarise, the comparison between results of global oscillation parameters from different methods has been very useful, because it allowed for the first time to provide a qualitative measure of the differences between different methods. The comparison has been used to investigate the contribution of the realization noise and internal uncertainties in the methods and allowed for the detection of artefacts. These issues will remain important and the comparison will continue to be applied. In the future, we will investigate the improvements in the accuracy of the results when we have data with longer timespan and investigate the actual uncertainties in the results.

Acknowledgements. Funding for the Kepler Mission is provided by NASA's Science Mission Directorate. The authors gratefully acknowledge the Kepler Science Team and all those who have contributed to making the Kepler Mission possible. S.H., Y.E., S.J.H. and W.J.C. acknowledge financial support from the UK Science and Technology Facilities Council (STFC). The research leading to these results has received funding from the European Research Council under the European Community's Seventh Framework Programme (FP7/20072013)/ERC grant agreement $n^{\circ} 227224$ (PROSPERITY), as well as from the Research Council of K.U.Leuven grant agreement GOA/2008/04. The National Center for Atmospheric Research is a federally funded research and development center sponsored by the US National Science Foundation. D.H. acknowledges support by the Astronomical Society of Australia (ASA).

\section{References}

Appourchaux, T., Michel, E., Auvergne, M., et al. 2008, A\&A, 488, 705 Baglin, A., Auvergne, M., Barge, P., et al. 2006, in ESA SP-1306, ed. M. Fridlund, A. Baglin, J. Lochard, \& L. Conroy, 33
Bedding, T. R., \& Kjeldsen, H. 2008, in 14th Cambridge Workshop on Cool Stars, Stellar Systems, and the Sun, ed. G. van Belle, ASP Conf. Ser., 384,21

Bedding, T. R., Huber, D., Stello, D., et al. 2010, ApJ, 713, L176

Benomar, O., Appourchaux, T., \& Baudin, F. 2009, A\&A, 506, 15

Borucki, W., Koch, D., Batalha, N., et al. 2009, in IAU Symp., 253, 289

Carrier, F., De Ridder, J., Baudin, F., et al. 2010, A\&A, 509, A73

Chaplin, W. J., \& Basu, S. 2008, Sol. Phys., 251, 53

De Ridder, J., Arentoft, T., \& Kjeldsen, H. 2006, MNRAS, 365, 595

De Ridder, J., Barban, C., Baudin, F., et al. 2009, Nature, 459, 398

Deheuvels, S., Bruntt, H., Michel, E., et al. 2010, A\&A, 515, A87

Gruberbauer, M., Kallinger, T., Weiss, W. W., \& Guenther, D. B. 2009, A\&A, 506,1043

Harvey, J. 1985, in Future Missions in Solar, Heliospheric \& Space Plasma Physics, ed. E. Rolfe, \& B. Battrick, ESA SP-235, 199

Hekker, S. 2010, Astron. Nachr., accepted [arXiv: 1006 . 4390]

Hekker, S., Kallinger, T., Baudin, F., et al. 2009, A\&A, 506, 465

Hekker, S., Broomhall, A., Chaplin, W. J., et al. 2010a, MNRAS, 402, 2049

Hekker, S., Debosscher, J., Huber, D., et al. 2010b, ApJ, 713, L187

Huber, D., Stello, D., Bedding, T. R., et al. 2009, Commun. Asteroseismol., 160, 74

Huber, D., Bedding, T. R., Stello, D., et al. 2010, ApJ, 723, 1607

Jenkins, J. M., Caldwell, D. A., Chandrasekaran, H., et al. 2010, ApJ, 713, L120

Kallinger, T., Mosser, B., Hekker, S., et al. 2010a, A\&A, 522, A1

Kallinger, T., Weiss, W. W., Barban, C., et al. 2010b, A\&A, 509, A77

Mathur, S., García, R. A., Régulo, C., et al. 2010, A\&A, 511, A46

Miglio, A., Montalbán, J., Baudin, F., et al. 2009, A\&A, 503, L21

Mosser, B., \& Appourchaux, T. 2009, A\&A, 508, 877

Mosser, B., Belkacem, K., Goupil, M., et al. 2010, A\&A, 517, A22

Stello, D., Chaplin, W. J., Basu, S., Elsworth, Y., \& Bedding, T. R. 2009, MNRAS, 400, L80

Stello, D., Basu, S., Bruntt, H., et al. 2010, ApJ, 713, L182

Tassoul, M. 1980, ApJS, 43, 469

Pages 12 to 31 are available in the electronic edition of the journal at http: //www . aanda. org 
A\&A 525, A131 (2011)

Table 7. Results for $v_{\max }$ and $\Delta v$ from different methods. Up-to-date results can be requested from the authors.

\begin{tabular}{|c|c|c|c|c|c|c|c|c|c|c|c|c|c|}
\hline Star & $\begin{array}{l}\mathrm{COR} \\
v_{\max } \\
\mu \mathrm{Hz}\end{array}$ & $\begin{array}{c}\text { COR } \\
\Delta v \\
\mu \mathrm{Hz}\end{array}$ & $\begin{array}{l}\text { CAN } \\
v_{\max } \\
\mu \mathrm{Hz}\end{array}$ & $\begin{array}{c}\text { CAN } \\
\Delta v \\
\mu \mathrm{Hz}\end{array}$ & $\begin{array}{l}\mathrm{A} 2 \mathrm{Z} \\
\nu_{\max } \\
\mu \mathrm{Hz}\end{array}$ & $\begin{array}{c}\mathrm{A} 2 \mathrm{Z} \\
\Delta v \\
\mu \mathrm{Hz}\end{array}$ & $\begin{array}{l}\text { SYD } \\
\nu_{\max } \\
\mu \mathrm{Hz}\end{array}$ & $\begin{array}{c}\text { SYD } \\
\Delta v \\
\mu \mathrm{Hz}\end{array}$ & $\begin{array}{c}\text { DLB } \\
v_{\max } \\
\mu \mathrm{Hz}\end{array}$ & $\begin{array}{c}\text { DLB } \\
\Delta v \\
\mu \mathrm{Hz}\end{array}$ & $\begin{array}{c}\text { OCT I } \\
v_{\max } \\
\mu \mathrm{Hz}\end{array}$ & $\begin{array}{c}\text { OCT II } \\
v_{\max } \\
\mu \mathrm{Hz}\end{array}$ & $\begin{array}{c}\text { OCT } \\
\Delta v \\
\mu \mathrm{Hz}\end{array}$ \\
\hline 1161618 & 33.50 & 4.22 & 33.58 & 4.15 & 33.79 & & 34.02 & 4.12 & 32.84 & 3.55 & 34.05 & 34.86 & 4.18 \\
\hline 1162746 & 27.20 & 3.80 & 26.39 & 4.10 & & & 27.68 & & & & 27.90 & 28.80 & \\
\hline 1432587 & 4.30 & 0.91 & & & & & & & & & & & \\
\hline 1433593 & 69.00 & 6.31 & 68.98 & 6.17 & 69.19 & 6.35 & 68.81 & 6.22 & & & 70.06 & 71.23 & 6.34 \\
\hline 1433730 & 41.40 & 4.12 & 41.58 & 4.16 & 38.17 & 4.03 & 41.45 & 4.16 & 37.57 & 4.38 & 42.02 & 44.73 & 4.11 \\
\hline 1435573 & 26.80 & 3.76 & 26.72 & 3.83 & & & 27.27 & 3.64 & & & 27.12 & 28.00 & 3.67 \\
\hline 1719297 & 7.60 & 1.19 & & & & & 7.59 & & & & & & \\
\hline 1720425 & 28.00 & 3.60 & & & 28.26 & 3.60 & 28.31 & 3.70 & 26.87 & 3.49 & 28.65 & 29.84 & 3.60 \\
\hline 1725552 & 7.90 & 1.29 & & & & & 7.92 & & 6.89 & & & & \\
\hline 1726211 & 30.30 & 3.72 & 30.20 & 3.78 & & & 30.54 & 3.77 & 25.60 & & 30.62 & 31.90 & 3.71 \\
\hline 1864183 & 54.50 & 5.59 & 56.03 & 5.54 & 54.46 & 5.59 & 54.38 & 5.56 & 52.81 & & 56.14 & 58.70 & 5.56 \\
\hline 1864855 & 129.90 & 10.42 & 129.32 & 10.40 & 128.16 & 10.43 & 127.46 & 10.41 & & & 129.07 & 132.56 & 10.51 \\
\hline 1868101 & 31.20 & 3.84 & 32.02 & 3.78 & & & 31.54 & & & & 32.06 & 33.63 & 3.80 \\
\hline 2013502 & 61.10 & 5.68 & 60.47 & 5.75 & 60.11 & 5.71 & 60.43 & 5.70 & & & 61.22 & 64.50 & 5.65 \\
\hline 2018392 & 33.40 & 3.78 & 34.07 & 3.74 & 33.10 & 3.79 & 33.72 & 3.78 & & & 34.61 & 34.39 & 3.75 \\
\hline 2142095 & 27.20 & 3.82 & 27.82 & 3.67 & 27.07 & 3.80 & 26.94 & 3.70 & & & & & \\
\hline 2156178 & 29.30 & 2.85 & & & 26.38 & 2.61 & 29.59 & 3.87 & & & & & \\
\hline 2300503 & 14.50 & 2.04 & & & 14.54 & 2.02 & 14.55 & & 14.83 & 1.63 & & & \\
\hline 2303367 & 34.60 & 4.04 & 33.67 & 3.93 & 32.07 & 3.98 & 34.82 & & 32.70 & 4.04 & 34.23 & 34.84 & 4.00 \\
\hline 2304765 & 81.10 & 6.31 & & & & & & & & & & & \\
\hline 2305930 & 29.50 & 3.32 & & & & & 29.70 & & & & 30.57 & 32.39 & 3.34 \\
\hline 2307683 & 40.60 & 4.09 & 40.55 & 4.22 & 40.84 & 4.15 & 40.35 & 4.35 & & & 40.86 & 42.70 & 4.24 \\
\hline 2309469 & 29.00 & 3.60 & 29.04 & 3.57 & & & 28.95 & 3.59 & & & 29.37 & 30.08 & 3.62 \\
\hline 2424822 & 94.30 & 8.18 & 92.92 & 8.04 & 93.49 & 8.18 & 92.91 & 8.08 & & & 93.82 & 92.22 & 8.16 \\
\hline 2424934 & 34.20 & 3.93 & 34.39 & 3.90 & 34.12 & 3.92 & 34.44 & 3.94 & 32.39 & 4.32 & 34.56 & 36.24 & 3.95 \\
\hline 2424955 & 50.80 & 5.37 & 50.64 & 5.42 & 50.26 & 5.50 & 51.27 & 5.34 & 45.65 & & 51.00 & 52.37 & 5.34 \\
\hline 2425631 & 21.20 & 2.57 & 21.32 & 2.58 & 21.28 & 2.50 & 21.41 & 2.62 & 17.61 & 2.92 & 21.68 & 22.52 & 2.57 \\
\hline 2444348 & 28.60 & 3.21 & 29.44 & 3.31 & 28.94 & 3.25 & 28.78 & 3.27 & 26.79 & & 29.58 & 31.58 & 3.22 \\
\hline 2448225 & 36.10 & 4.09 & 35.85 & 4.08 & 36.31 & 3.97 & 36.17 & 4.08 & 34.94 & 4.37 & 35.98 & 37.39 & 4.08 \\
\hline 2449703 & 29.00 & 3.95 & 30.39 & 3.95 & & & 28.53 & 3.89 & & & 30.54 & 32.15 & 3.96 \\
\hline 2554924 & 36.00 & 4.04 & 36.22 & 4.04 & & & 36.00 & & & & 36.39 & 37.39 & 4.14 \\
\hline 2573092 & 36.50 & 4.22 & 37.20 & 4.41 & 36.66 & 4.23 & 35.06 & 4.10 & & & 37.69 & 38.00 & 4.16 \\
\hline 2695975 & 10.20 & 1.53 & & & & & 10.52 & & 9.23 & & & & \\
\hline 2696628 & 10.60 & 1.51 & & & & & 10.57 & & & & & & \\
\hline 2696732 & 90.70 & 8.28 & 90.20 & 8.04 & 90.56 & 8.46 & 90.96 & 8.28 & & & 90.40 & 91.02 & 8.30 \\
\hline 2696955 & 107.90 & 8.38 & 109.16 & 8.63 & & & 107.26 & 8.65 & & & 108.59 & 110.39 & 8.56 \\
\hline 2714397 & 31.80 & 4.14 & 32.39 & 4.26 & 34.37 & 3.97 & 33.26 & 4.22 & 33.99 & 4.51 & 32.62 & 33.58 & 4.11 \\
\hline 2831788 & 54.90 & 5.54 & 55.11 & 5.48 & 54.96 & 5.67 & 55.13 & 5.48 & 54.32 & 5.25 & 55.21 & 56.62 & 5.54 \\
\hline 2847978 & 9.50 & 1.60 & & & & & 9.63 & & & & & & \\
\hline 2988638 & 82.30 & 7.39 & 85.63 & 7.47 & 85.10 & 7.42 & 84.01 & 7.46 & & & 88.78 & 92.08 & 7.42 \\
\hline 2988988 & 38.70 & 4.77 & & & 38.81 & 4.54 & 38.52 & & 36.38 & 4.50 & 38.94 & 39.97 & 5.16 \\
\hline 2995197 & 9.60 & 1.56 & & & & & 9.77 & & & & & & \\
\hline 3098045 & 33.20 & 3.66 & 34.57 & 3.66 & 34.26 & 3.23 & 33.70 & & & & 34.60 & 36.28 & 3.65 \\
\hline 3098716 & 30.60 & 4.41 & 31.26 & 3.74 & & & 30.73 & & & & 31.27 & 32.05 & 4.27 \\
\hline 3100193 & 28.10 & 3.56 & 29.01 & 3.45 & 28.67 & 3.45 & 27.44 & 3.50 & 24.56 & 3.40 & 29.32 & 31.39 & 3.36 \\
\hline 3101090 & 30.80 & 3.88 & 31.11 & 4.06 & 30.36 & 3.83 & 30.53 & 3.91 & 29.27 & & 31.17 & 33.22 & 3.93 \\
\hline 3110460 & 7.80 & 1.46 & & & & & 8.32 & & & & & & \\
\hline 3114408 & 30.50 & 3.42 & 29.83 & 3.43 & 29.54 & 3.42 & 31.03 & 3.42 & & & 30.44 & 31.51 & 3.38 \\
\hline 3118806 & 19.00 & 2.63 & 19.36 & 2.82 & 17.89 & 1.90 & 19.25 & 2.72 & 19.23 & 2.02 & 19.74 & 22.78 & 2.61 \\
\hline 3120486 & & & & & 137.53 & 10.75 & 137.06 & 10.60 & & & & & \\
\hline 3120585 & 100.40 & 8.49 & 97.97 & 8.44 & 98.98 & 8.46 & 99.00 & 8.49 & & & 100.88 & 102.90 & 8.39 \\
\hline 3121940 & 27.90 & 3.74 & 27.33 & 3.81 & 27.57 & 3.64 & 28.68 & & & & 28.21 & 30.05 & 3.72 \\
\hline 3217051 & 34.10 & 4.27 & 33.90 & 4.41 & & & 33.92 & & & & 34.83 & 37.37 & 4.25 \\
\hline 3231503 & 42.90 & 4.30 & 43.04 & 4.23 & 42.41 & 4.37 & 42.93 & 4.30 & 40.71 & 4.62 & 42.94 & 43.39 & 4.28 \\
\hline 3323943 & 32.60 & 4.17 & 32.67 & 4.21 & 31.29 & 4.11 & 32.98 & 4.11 & & & 33.19 & 34.52 & 4.16 \\
\hline 3326790 & 24.30 & 3.62 & & & 24.60 & 3.62 & 24.99 & 3.53 & 25.57 & 3.28 & 26.44 & 26.37 & 3.61 \\
\hline 3339894 & 31.10 & 3.34 & 29.77 & 3.17 & 28.27 & 3.17 & 31.40 & 3.22 & & & 31.61 & 34.28 & 3.20 \\
\hline 3346501 & 19.80 & 2.49 & 20.71 & 2.49 & & & 19.94 & & & & 20.61 & 22.17 & \\
\hline 3355015 & 26.50 & 3.45 & 26.82 & 3.45 & & & 26.65 & & & & 27.42 & 28.75 & 3.45 \\
\hline 3425476 & 37.10 & 4.49 & 37.77 & 4.96 & 38.85 & 4.44 & 36.77 & & & & 38.53 & 42.10 & 4.48 \\
\hline 3426898 & 62.90 & 6.61 & 63.61 & 6.54 & 62.73 & 6.62 & 62.88 & 6.54 & & & 63.68 & 65.79 & 6.52 \\
\hline 3443483 & & & & & 134.77 & 10.87 & 132.06 & 10.70 & & & 134.44 & 133.26 & 10.75 \\
\hline 3444374 & 56.20 & 5.73 & 56.92 & 5.71 & 55.96 & 5.68 & 56.17 & 5.72 & & & 56.51 & 58.95 & 5.74 \\
\hline 3455760 & 47.80 & 4.88 & 48.18 & 4.87 & 48.10 & 4.87 & 47.75 & 4.88 & 44.45 & & 48.13 & 51.18 & 4.82 \\
\hline 3457618 & 37.30 & 3.64 & & & 34.31 & 3.59 & 37.17 & 3.61 & & & 36.61 & 36.19 & 3.61 \\
\hline
\end{tabular}


Table 7. continued.

\begin{tabular}{|c|c|c|c|c|c|c|c|c|c|c|c|c|c|}
\hline Star & $\begin{array}{l}\mathrm{COR} \\
v_{\max } \\
\mu \mathrm{Hz}\end{array}$ & $\begin{array}{c}\mathrm{COR} \\
\Delta v \\
\mu \mathrm{Hz}\end{array}$ & $\begin{array}{l}\text { CAN } \\
v_{\max } \\
\mu \mathrm{Hz}\end{array}$ & $\begin{array}{c}\text { CAN } \\
\Delta v \\
\mu \mathrm{Hz}\end{array}$ & $\begin{array}{l}\mathrm{A} 2 \mathrm{Z} \\
v_{\max } \\
\mu \mathrm{Hz}\end{array}$ & $\begin{array}{c}\mathrm{A} 2 \mathrm{Z} \\
\Delta v \\
\mu \mathrm{Hz}\end{array}$ & $\begin{array}{l}\text { SYD } \\
v_{\max } \\
\mu \mathrm{Hz}\end{array}$ & $\begin{array}{c}\text { SYD } \\
\Delta v \\
\mu \mathrm{Hz}\end{array}$ & $\begin{array}{l}\text { DLB } \\
v_{\max } \\
\mu \mathrm{Hz}\end{array}$ & $\begin{array}{c}\text { DLB } \\
\Delta v \\
\mu \mathrm{Hz}\end{array}$ & $\begin{array}{c}\text { OCT I } \\
v_{\max } \\
\mu \mathrm{Hz}\end{array}$ & $\begin{array}{c}\text { OCT II } \\
v_{\max } \\
\mu \mathrm{Hz}\end{array}$ & $\begin{array}{c}\mathrm{OCT} \\
\Delta v \\
\mu \mathrm{Hz}\end{array}$ \\
\hline 3526061 & 127.10 & 10.74 & 129.21 & 10.59 & 129.28 & 10.00 & 128.96 & 10.67 & 126.04 & 11.07 & 129.24 & 130.04 & 10.68 \\
\hline 3528656 & 19.60 & 2.66 & 20.68 & 2.66 & 19.50 & 2.67 & 20.16 & 2.69 & & & 21.17 & 22.26 & 2.69 \\
\hline 3529276 & 57.50 & 5.83 & 56.79 & 5.63 & 57.44 & 5.74 & 58.15 & 5.66 & 54.38 & 6.27 & 57.14 & 57.96 & 5.84 \\
\hline 3530520 & 37.60 & 3.97 & 36.52 & 3.90 & 34.65 & 3.97 & 37.78 & 3.97 & & & 36.84 & 36.86 & 3.93 \\
\hline 3530823 & 43.90 & 4.64 & 44.41 & 4.72 & 43.24 & 4.61 & 43.85 & & & & 44.06 & 46.62 & 4.67 \\
\hline 3531478 & & & & & 236.91 & 17.38 & 241.15 & 17.19 & & & & & 17.51 \\
\hline 3560181 & 34.10 & 3.95 & 33.78 & 3.89 & 32.85 & 3.95 & 34.35 & 3.88 & 32.81 & 3.64 & 34.09 & 34.73 & 3.93 \\
\hline 3631092 & 195.60 & 14.03 & & & & & 197.43 & 13.97 & & & 200.17 & 196.91 & 14.13 \\
\hline 3631402 & 48.30 & 5.02 & 46.38 & 5.28 & 47.16 & 4.90 & 48.45 & 4.93 & & & 47.43 & 49.04 & 5.09 \\
\hline 3642038 & 41.60 & 4.41 & 41.99 & 4.33 & 38.63 & 4.33 & 42.51 & 4.27 & 40.09 & & 42.22 & 42.62 & 4.34 \\
\hline 3645232 & 7.80 & 1.45 & & & & & 8.12 & 1.42 & & & & & \\
\hline 3656231 & 37.20 & 4.27 & 37.46 & 4.23 & 37.06 & 4.25 & 37.27 & 4.26 & & & 37.74 & 39.53 & 4.29 \\
\hline 3658136 & 7.40 & 1.32 & & & & & 7.32 & & & & & & \\
\hline 3658812 & & & 16.94 & 2.38 & & & 18.70 & & & & & & \\
\hline 3660820 & & & & & 33.27 & 4.04 & 32.49 & 4.01 & & & & & \\
\hline 3660976 & 39.40 & 3.88 & & & 30.46 & 3.89 & 39.52 & 3.86 & & & 37.80 & 38.47 & 3.86 \\
\hline 3729799 & 38.10 & 4.12 & 38.04 & 4.19 & 38.38 & 4.10 & 38.31 & 4.16 & & & 38.79 & 39.20 & 4.14 \\
\hline 3730953 & 48.30 & 4.95 & 47.89 & 5.03 & 48.09 & 4.89 & 48.75 & 5.01 & 46.37 & 5.05 & 48.32 & 49.25 & 4.92 \\
\hline 3744043 & 111.80 & 9.96 & 110.93 & 9.82 & 111.24 & 9.78 & 111.57 & 9.85 & 107.68 & & & & \\
\hline 3747777 & 44.10 & 4.43 & 43.15 & 4.42 & 42.75 & 4.50 & 43.75 & 4.43 & 33.81 & & 43.22 & 46.18 & 4.43 \\
\hline 3747860 & 36.50 & 4.14 & 36.51 & 4.18 & 35.44 & 4.03 & 36.92 & 4.10 & & & 37.06 & 37.55 & 4.00 \\
\hline 3748585 & 44.60 & 4.64 & 44.02 & 4.60 & 44.41 & 4.74 & 44.83 & 4.63 & 42.35 & & 44.98 & 48.10 & 4.65 \\
\hline 3748691 & 38.80 & 4.17 & 39.72 & 4.34 & 39.92 & 4.21 & 38.94 & & & & 39.67 & 41.34 & 4.22 \\
\hline 3758458 & 74.10 & 5.93 & 72.54 & 6.04 & 72.19 & 5.90 & 73.00 & 5.83 & 71.96 & & 73.54 & 76.16 & 5.94 \\
\hline 3760337 & 38.20 & 4.22 & 38.63 & 4.25 & 38.29 & 4.15 & 38.33 & 4.26 & 36.24 & 4.30 & 39.00 & 40.81 & 4.18 \\
\hline 3835996 & 45.10 & 4.98 & 43.34 & 5.09 & 44.09 & 4.86 & 45.20 & 5.01 & & & 44.95 & 45.66 & 4.96 \\
\hline 3839310 & 12.80 & 1.52 & & & & & 13.14 & & & & & & \\
\hline 3842486 & 11.20 & 1.72 & & & 11.36 & 1.62 & 11.38 & & & & & & \\
\hline 3842704 & 7.20 & 1.00 & & & & & & & & & & & \\
\hline 3847071 & 58.70 & 6.08 & 61.13 & 6.14 & 59.54 & 5.96 & 58.84 & 6.13 & & & 60.94 & 63.28 & 6.12 \\
\hline 3847697 & 48.10 & 4.84 & 46.78 & 4.87 & 46.48 & 4.86 & 48.64 & 4.92 & & & 46.95 & 47.89 & 4.88 \\
\hline 3849996 & 26.90 & 2.48 & 26.38 & 3.72 & & & 27.88 & 4.00 & & & 27.53 & 29.18 & \\
\hline 3854605 & 37.20 & 4.17 & 37.78 & 4.29 & 37.33 & 4.22 & 37.58 & 4.16 & 37.09 & 4.45 & 37.95 & 39.96 & 4.18 \\
\hline 3858714 & 46.40 & 5.05 & 47.49 & 5.05 & 46.20 & 5.00 & 46.22 & 4.99 & & & 47.80 & 49.65 & 4.85 \\
\hline 3860139 & 20.40 & 2.58 & 21.17 & 2.60 & 19.29 & 2.52 & 20.64 & 2.56 & 15.90 & 2.08 & & & \\
\hline 3860176 & 76.70 & 6.67 & 71.44 & 6.85 & 71.04 & 6.67 & 78.86 & 6.64 & & & 76.11 & 74.30 & 6.61 \\
\hline 3867193 & 49.50 & 4.91 & 49.09 & 4.95 & 48.63 & 5.00 & 49.68 & 4.93 & & & 48.79 & 47.56 & 4.88 \\
\hline 3869326 & 47.60 & 4.68 & 47.79 & 4.63 & 47.12 & 4.62 & 48.52 & 4.62 & & & 47.62 & 48.20 & 4.63 \\
\hline 3935987 & 20.10 & 2.54 & 20.53 & 2.57 & 19.93 & 2.70 & 20.24 & 2.58 & & & 20.58 & 21.68 & 2.59 \\
\hline 3936921 & 27.10 & 3.35 & 27.50 & 3.70 & & & 26.99 & & 26.36 & 3.25 & 27.69 & 29.14 & \\
\hline 3937217 & & & & & 27.59 & 3.55 & 29.14 & & & & & & \\
\hline 3941538 & 24.30 & 3.58 & & & 24.27 & 2.50 & 26.32 & & & & & & \\
\hline 3946389 & 23.10 & 2.83 & 22.91 & 2.95 & 22.96 & 2.38 & 23.26 & 2.94 & & & 23.54 & 24.82 & 2.78 \\
\hline 3955590 & 18.40 & 2.74 & & & 18.13 & 2.99 & 18.80 & 2.76 & & & 19.13 & 20.93 & 2.77 \\
\hline 4036007 & 38.10 & 4.41 & 38.16 & 4.50 & & & 38.03 & & & & 38.65 & 40.40 & 4.39 \\
\hline $\begin{array}{l}4039306 \\
4039831\end{array}$ & 34.00 & 4.04 & 32.83 & 4.04 & & & $\begin{array}{c}35.48 \\
6.18\end{array}$ & & & & 33.73 & 34.31 & 4.14 \\
\hline 4042951 & 31.60 & 4.32 & 31.98 & 4.23 & & & 31.57 & 4.22 & & & 32.31 & 34.36 & 4.29 \\
\hline 4044238 & 33.00 & 4.17 & 33.03 & 4.19 & 32.96 & 3.90 & 32.46 & 4.24 & & & 33.92 & 34.97 & 4.14 \\
\hline 4056266 & 86.30 & 7.39 & 86.61 & 7.47 & 86.25 & 7.35 & 85.46 & 7.42 & & & 87.30 & 90.17 & 7.40 \\
\hline 4057076 & 41.30 & 4.55 & 40.96 & 4.99 & 40.36 & 4.58 & 41.64 & 4.53 & & & 41.66 & 43.36 & 4.53 \\
\hline 4057744 & 26.40 & 2.96 & 26.11 & 2.83 & 25.23 & 2.85 & 26.52 & 2.97 & 23.74 & 3.67 & 26.48 & 26.28 & \\
\hline 4069447 & & & & & & & 7.87 & 1.30 & & & & & \\
\hline 4140726 & 50.30 & 4.77 & 50.54 & 4.96 & 50.28 & 5.00 & 51.12 & 4.98 & & & 50.77 & 51.16 & 4.83 \\
\hline 4155395 & & & & & & & 4.97 & & & & & & \\
\hline 4157282 & 15.00 & 1.95 & & & 15.21 & 1.92 & 15.26 & 1.92 & 15.69 & & & & \\
\hline 4164910 & 36.30 & 3.91 & 37.01 & 4.47 & 37.13 & 4.20 & 36.32 & 4.17 & 32.34 & 4.35 & 37.59 & 39.89 & 3.98 \\
\hline 4169130 & 37.00 & 4.35 & 37.70 & 4.69 & 37.23 & & 37.33 & 4.31 & & & 38.00 & 39.03 & 4.36 \\
\hline 4243796 & 37.50 & 4.35 & & & 37.57 & 4.27 & 37.56 & 4.28 & 36.68 & 4.57 & 38.01 & 39.81 & 4.30 \\
\hline 4243803 & & & & & & & 16.83 & & 14.86 & 2.27 & & & \\
\hline 4244973 & 50.60 & 4.74 & 50.53 & & 51.01 & 4.91 & 51.77 & 4.74 & 44.91 & 4.99 & 51.32 & 52.30 & 4.74 \\
\hline 4249128 & 42.00 & 4.12 & 40.88 & 4.56 & 41.56 & 4.45 & 42.09 & 4.48 & & & 41.38 & 43.17 & 4.49 \\
\hline 4249357 & 47.60 & 5.09 & 47.00 & 5.17 & 47.29 & 5.17 & 47.63 & 5.17 & & & 47.97 & 47.47 & 5.04 \\
\hline 4262505 & 88.20 & 7.99 & 89.15 & 7.94 & 88.82 & 8.03 & 87.94 & 7.97 & 88.71 & & 89.36 & 90.21 & 8.01 \\
\hline 4269391 & 39.60 & 4.19 & 35.80 & 4.11 & 37.20 & 4.09 & 40.23 & 4.22 & 33.10 & & 37.54 & 36.81 & 4.08 \\
\hline
\end{tabular}


Table 7. continued.

\begin{tabular}{|c|c|c|c|c|c|c|c|c|c|c|c|c|c|}
\hline Star & $\begin{array}{c}\text { COR } \\
v_{\max } \\
\mu \mathrm{Hz}\end{array}$ & $\begin{array}{c}\text { COR } \\
\Delta v \\
\mu \mathrm{Hz}\end{array}$ & $\begin{array}{l}\text { CAN } \\
v_{\max } \\
\mu \mathrm{Hz}\end{array}$ & $\begin{array}{c}\text { CAN } \\
\Delta v \\
\mu \mathrm{Hz}\end{array}$ & $\begin{array}{l}\mathrm{A} 2 \mathrm{Z} \\
v_{\max } \\
\mu \mathrm{Hz}\end{array}$ & $\begin{array}{l}\mathrm{A} 2 \mathrm{Z} \\
\Delta v \\
\mu \mathrm{Hz}\end{array}$ & $\begin{array}{l}\text { SYD } \\
v_{\max } \\
\mu \mathrm{Hz}\end{array}$ & $\begin{array}{c}\text { SYD } \\
\Delta v \\
\mu \mathrm{Hz}\end{array}$ & $\begin{array}{c}\text { DLB } \\
v_{\max } \\
\mu \mathrm{Hz}\end{array}$ & $\begin{array}{c}\text { DLB } \\
\Delta v \\
\mu \mathrm{Hz}\end{array}$ & $\begin{array}{c}\text { OCT I } \\
v_{\max } \\
\mu \mathrm{Hz}\end{array}$ & $\begin{array}{c}\text { OCT II } \\
v_{\max } \\
\mu \mathrm{Hz}\end{array}$ & $\begin{array}{c}\text { OCT } \\
\Delta v \\
\mu \mathrm{Hz}\end{array}$ \\
\hline 4271039 & 46.40 & 5.37 & 47.05 & 5.16 & 46.14 & 5.11 & 46.54 & 5.21 & & & 46.90 & 48.07 & 5.08 \\
\hline 4275625 & 31.30 & 3.84 & & & 31.23 & 3.83 & 31.13 & 3.82 & 31.69 & 3.41 & 31.94 & 34.03 & 3.77 \\
\hline 4276070 & & & & & 42.33 & 4.52 & 44.98 & 4.52 & & & 45.73 & 47.87 & 4.59 \\
\hline 4279700 & 31.10 & 3.56 & 32.07 & 3.56 & 31.10 & 3.64 & 31.00 & 3.53 & & & 32.07 & 34.26 & 3.52 \\
\hline 4281702 & 30.10 & 3.95 & 29.86 & 4.06 & 31.26 & 3.87 & 29.97 & 3.97 & & & & & \\
\hline 4283484 & 34.30 & 4.27 & & & & & 28.95 & & 32.84 & 4.35 & & & \\
\hline 4345370 & 32.40 & 4.07 & 31.40 & 4.07 & 32.08 & & 32.99 & 4.04 & & & 32.27 & 32.69 & \\
\hline 4350501 & & & & & 150.00 & 10.95 & 138.94 & 11.05 & & & 140.06 & 143.59 & 11.00 \\
\hline 4365609 & 34.30 & 3.97 & 34.41 & 4.21 & & & 35.11 & 4.18 & & & 35.10 & 36.57 & \\
\hline 4365827 & & & & & & & & & 1.32 & 0.40 & & & \\
\hline 4374106 & 14.70 & 2.10 & & & 14.26 & 2.12 & 15.04 & 2.09 & 13.46 & 1.92 & 15.21 & 16.18 & 2.10 \\
\hline 4378473 & 22.60 & 2.69 & 23.04 & 2.82 & 22.31 & 2.74 & 22.71 & 2.84 & 23.00 & 2.83 & 22.99 & 23.93 & \\
\hline 4383388 & 42.30 & 4.88 & 42.47 & 4.87 & 42.49 & 4.81 & 41.69 & 4.83 & 39.49 & & 44.33 & 45.10 & 4.82 \\
\hline 4445711 & 35.70 & 4.24 & 34.75 & 4.29 & 34.63 & 4.18 & 35.63 & & 32.79 & & 35.70 & 36.29 & 4.21 \\
\hline 4446181 & 30.20 & 4.17 & & & 28.74 & 4.12 & 30.70 & 4.20 & 30.22 & 3.84 & & & \\
\hline 4448636 & 31.50 & 4.09 & 32.20 & 4.36 & & & 31.89 & & & & 32.86 & 33.62 & 4.04 \\
\hline 4448777 & 206.90 & 17.19 & 221.87 & 16.90 & 220.00 & 17.12 & 219.47 & 17.02 & & & & & 16.99 \\
\hline 4456739 & 36.80 & 4.22 & 36.26 & 4.30 & 36.82 & 4.21 & 38.16 & 4.22 & & & 37.14 & 39.41 & 4.19 \\
\hline 4457200 & 44.90 & 4.52 & 43.31 & 4.38 & 43.78 & 4.62 & 45.09 & 4.39 & 45.00 & 4.51 & 43.81 & 45.26 & 4.53 \\
\hline 4457395 & 34.00 & 4.14 & 33.92 & 4.05 & 33.16 & 3.85 & 34.37 & 4.06 & & & 34.44 & 34.59 & 4.21 \\
\hline 4469602 & 33.60 & 3.53 & 33.47 & 3.62 & 31.75 & 3.50 & 33.88 & 3.56 & 30.49 & 3.92 & 33.65 & 34.75 & 3.48 \\
\hline 4469886 & 58.30 & 5.50 & 55.64 & 5.24 & 55.63 & 5.54 & 58.84 & 5.45 & & & 57.52 & 56.23 & 5.52 \\
\hline 4475934 & 39.90 & 4.43 & 39.85 & 4.56 & 39.88 & 4.51 & 40.43 & 4.41 & & & 40.20 & 41.63 & 4.43 \\
\hline 4479314 & 18.80 & 2.28 & & & & & 19.28 & 2.24 & 17.54 & 2.52 & & & \\
\hline 4480358 & 8.10 & 1.53 & & & & & 8.21 & & & & & & \\
\hline 4480938 & 34.20 & 4.04 & 35.19 & 4.32 & 34.57 & 3.92 & 34.15 & 4.03 & & & 35.36 & 37.53 & 3.97 \\
\hline 4489185 & 94.20 & 8.59 & 94.65 & 8.55 & 94.15 & 8.45 & 93.28 & 8.59 & & & 94.69 & 98.39 & 8.61 \\
\hline 4545474 & 37.40 & 3.88 & 37.33 & 3.74 & 38.02 & 3.92 & 37.69 & 3.90 & 37.79 & & 38.48 & 39.67 & 3.88 \\
\hline 4546625 & 32.50 & 4.17 & 33.42 & 4.32 & & & 33.03 & 4.25 & & & 34.29 & 36.04 & 4.16 \\
\hline $\begin{array}{l}4548564 \\
4548615\end{array}$ & 58.40 & 6.03 & 58.49 & 5.99 & 57.97 & 6.00 & $\begin{array}{c}58.66 \\
3.52\end{array}$ & 6.00 & & & 58.64 & 58.96 & 6.03 \\
\hline 4553892 & 44.00 & 4.64 & 44.26 & 4.63 & 43.18 & 4.71 & 44.21 & 4.62 & & & 44.34 & 44.35 & 4.64 \\
\hline 4555456 & 7.90 & 1.36 & & & & & 7.97 & & & & & & \\
\hline 4567221 & 13.00 & 1.81 & & & & & 12.91 & 1.80 & 13.24 & 2.14 & & & \\
\hline 4570120 & 88.50 & 7.31 & 88.33 & 7.54 & 87.31 & 7.33 & 88.78 & 7.34 & & & 88.93 & 91.66 & 7.38 \\
\hline 4571402 & 37.40 & 4.27 & 37.20 & 4.43 & 36.94 & 4.26 & 37.33 & & 34.52 & 4.41 & 37.45 & 39.44 & 4.20 \\
\hline 4571962 & 7.40 & 1.07 & & & 8.63 & 1.04 & 7.40 & & & & & & \\
\hline 4575645 & 38.30 & 4.43 & 39.86 & 4.29 & 39.57 & 4.49 & 37.93 & 4.31 & 38.08 & 4.28 & 40.24 & 42.38 & 4.43 \\
\hline 4578488 & 47.90 & 5.33 & 48.83 & 5.29 & 47.66 & 5.26 & 47.71 & 5.32 & & & 48.55 & 51.07 & 5.28 \\
\hline 4633907 & 30.50 & 3.93 & 30.76 & 3.88 & & & 30.80 & 3.94 & 29.95 & & 30.99 & 32.02 & 3.98 \\
\hline 4636731 & 23.40 & 2.96 & 22.88 & 3.03 & 23.03 & 3.19 & 23.82 & 2.91 & & & 23.81 & 24.83 & 2.96 \\
\hline 4638317 & 28.60 & 3.40 & 28.87 & 3.58 & 28.23 & 2.87 & 29.03 & 3.48 & 29.46 & 3.68 & 29.04 & 30.49 & 3.43 \\
\hline 4640537 & 34.80 & 3.95 & 34.61 & 3.97 & 34.01 & 3.98 & 35.08 & 4.03 & & & 34.97 & 35.86 & 3.97 \\
\hline 4644949 & 62.60 & 6.25 & 59.02 & 6.20 & 59.49 & 6.25 & 63.79 & 6.29 & & & 60.21 & 60.54 & 6.21 \\
\hline 4645692 & 34.00 & 4.12 & 34.06 & 3.92 & & & 35.16 & & & & & & \\
\hline 4646245 & 40.00 & 4.58 & 41.30 & 4.64 & & & 39.73 & 4.71 & 38.45 & 4.26 & 41.99 & 45.32 & 4.57 \\
\hline 4651366 & 54.30 & 4.64 & 54.32 & 4.51 & 53.93 & 4.70 & 54.04 & & & & 55.20 & 56.38 & 4.68 \\
\hline 4659706 & 33.50 & 3.70 & 34.36 & 3.78 & 35.59 & 3.75 & 34.51 & 3.81 & 30.18 & & 35.08 & 37.59 & 3.73 \\
\hline 4662188 & 16.00 & 2.18 & 16.53 & 2.20 & 15.73 & 2.12 & 16.43 & 2.14 & & & 16.63 & 17.14 & \\
\hline 4662939 & 65.00 & 5.46 & 61.80 & 5.46 & 62.29 & 5.48 & 65.03 & 5.52 & 61.04 & 5.15 & 64.32 & 68.72 & 5.53 \\
\hline 4672904 & 57.90 & 5.93 & 58.63 & 6.09 & 57.87 & 5.95 & 58.05 & 6.12 & & & 58.55 & 60.61 & 5.92 \\
\hline 4726049 & & & & & 253.12 & 16.86 & 249.24 & 17.85 & & & & & \\
\hline 4736611 & 90.60 & 7.99 & 91.39 & 7.97 & & & 90.63 & 7.99 & & & 91.95 & 95.57 & 7.95 \\
\hline 4738255 & 15.00 & 2.24 & 15.24 & 2.31 & 15.55 & 2.07 & 15.34 & 2.27 & & & & & \\
\hline 4738610 & & & & & & & 8.69 & & & & & & \\
\hline 4739728 & 27.70 & 3.62 & 28.53 & 3.83 & 27.88 & 3.44 & 27.41 & 3.53 & 25.07 & 3.04 & 28.52 & 29.58 & 3.58 \\
\hline 4743924 & 102.20 & 8.09 & 102.58 & & 103.21 & 8.00 & 101.13 & 8.20 & 95.63 & 9.27 & 105.67 & 108.50 & 8.05 \\
\hline 4750456 & 56.60 & 5.88 & 56.65 & 5.89 & 56.60 & 5.82 & 56.83 & 5.91 & & & 56.74 & 58.35 & 5.78 \\
\hline $\begin{array}{l}4755467 \\
4756934\end{array}$ & 38.00 & 4.43 & 36.85 & 4.32 & & & $\begin{array}{l}38.55 \\
10.37\end{array}$ & & & & 37.88 & 37.83 & 4.43 \\
\hline $\begin{array}{l}4 / 56934 \\
4760954\end{array}$ & 43.60 & 4.81 & 43.55 & 4.89 & 43.97 & 4.91 & 42.73 & 4.79 & & & 45.01 & 46.88 & 4.85 \\
\hline 4764420 & 37.30 & 4.27 & 37.96 & 4.48 & & & 37.09 & & & & 38.37 & 41.16 & 4.22 \\
\hline 4770846 & 54.00 & 5.37 & 54.65 & 5.51 & 55.37 & 5.30 & 53.99 & 5.47 & 52.86 & 5.79 & 55.39 & 57.48 & \\
\hline 4771707 & 6.30 & 1.03 & & & & & & & & & & & \\
\hline 4773539 & 42.10 & 4.52 & 42.64 & 4.56 & 41.95 & 4.50 & 42.41 & 4.52 & & & 42.90 & 44.67 & 4.54 \\
\hline
\end{tabular}


S. Hekker et al.: Comparison of global oscillation parameters from different methods

Table 7. continued.

\begin{tabular}{|c|c|c|c|c|c|c|c|c|c|c|c|c|c|}
\hline Star & $\begin{array}{l}\mathrm{COR} \\
v_{\max } \\
\mu \mathrm{Hz}\end{array}$ & $\begin{array}{c}\text { COR } \\
\Delta v \\
\mu \mathrm{Hz}\end{array}$ & $\begin{array}{l}\text { CAN } \\
v_{\max } \\
\mu \mathrm{Hz}\end{array}$ & $\begin{array}{c}\text { CAN } \\
\Delta v \\
\mu \mathrm{Hz}\end{array}$ & $\begin{array}{l}\mathrm{A} 2 \mathrm{Z} \\
\nu_{\max } \\
\mu \mathrm{Hz}\end{array}$ & $\begin{array}{c}\mathrm{A} 2 \mathrm{Z} \\
\Delta v \\
\mu \mathrm{Hz}\end{array}$ & $\begin{array}{l}\text { SYD } \\
v_{\max } \\
\mu \mathrm{Hz}\end{array}$ & $\begin{array}{c}\text { SYD } \\
\Delta v \\
\mu \mathrm{Hz}\end{array}$ & $\begin{array}{c}\text { DLB } \\
v_{\max } \\
\mu \mathrm{Hz}\end{array}$ & $\begin{array}{c}\text { DLB } \\
\Delta v \\
\mu \mathrm{Hz}\end{array}$ & $\begin{array}{c}\text { OCT I } \\
v_{\max } \\
\mu \mathrm{Hz}\end{array}$ & $\begin{array}{c}\text { OCT II } \\
v_{\max } \\
\mu \mathrm{Hz}\end{array}$ & $\begin{array}{c}\text { OCT } \\
\Delta v \\
\mu \mathrm{Hz}\end{array}$ \\
\hline 4813971 & 34.70 & 4.02 & 33.85 & 4.08 & 33.34 & 3.87 & 35.11 & 4.01 & & & 34.84 & 36.18 & 3.93 \\
\hline 4818830 & 41.30 & 4.64 & 41.88 & 4.73 & & & 41.40 & 4.69 & & & 41.83 & 44.83 & 4.60 \\
\hline 4821839 & 19.30 & 2.40 & & & 19.66 & 2.39 & 19.64 & 2.32 & 18.21 & 2.88 & 19.37 & 19.91 & 2.40 \\
\hline 4828670 & 43.20 & 4.43 & 44.14 & 5.19 & 42.18 & 4.42 & 43.52 & 4.50 & 45.84 & 5.00 & & & \\
\hline 4843143 & 33.40 & 4.00 & 33.63 & 3.89 & 33.48 & 3.94 & 33.78 & 3.93 & 29.04 & & 34.05 & 35.74 & 3.93 \\
\hline 4845272 & 31.00 & 3.56 & 31.48 & 3.57 & 30.51 & 3.62 & 31.22 & 3.61 & 26.51 & & 31.31 & 32.27 & 3.54 \\
\hline 4857853 & 30.50 & 3.91 & & & & & 31.23 & 3.88 & & & 31.10 & 32.50 & 3.96 \\
\hline 4902641 & 97.40 & 7.90 & 97.30 & 7.85 & 97.82 & 8.00 & 98.14 & 7.89 & 97.37 & & 97.75 & 102.80 & 7.90 \\
\hline 4902701 & 35.20 & 4.14 & 35.15 & 4.10 & & & 35.38 & 4.10 & & & 35.12 & 35.91 & \\
\hline 4908025 & 7.40 & 1.21 & & & 7.67 & 1.20 & 7.67 & & & & & & \\
\hline 4920044 & 9.60 & 1.73 & & & 9.41 & 1.64 & 9.87 & & 9.57 & 1.72 & & & \\
\hline 4930906 & 31.70 & 3.84 & 31.77 & 4.15 & & & 31.45 & 3.92 & & & 32.73 & 33.98 & 3.78 \\
\hline 4933594 & 33.80 & 4.07 & 34.62 & 4.27 & 34.48 & 4.08 & 33.73 & 4.14 & & & 34.89 & 37.13 & 4.10 \\
\hline 4937204 & 39.40 & 4.46 & 40.01 & 4.46 & 39.31 & 4.44 & 39.51 & 4.39 & 39.15 & 4.81 & 40.12 & 42.25 & 4.47 \\
\hline 4937576 & & & 32.21 & 3.59 & & & & & & & & & \\
\hline 4946220 & 23.40 & 2.77 & & & & & & & & & & & \\
\hline 4949422 & 52.60 & 4.68 & 50.74 & 4.63 & 49.40 & 4.67 & 52.40 & 4.61 & 41.15 & & 50.98 & 50.29 & 4.72 \\
\hline 4950323 & 57.10 & 6.03 & 58.39 & 5.98 & 56.46 & 6.08 & 56.74 & 5.88 & & & 58.33 & 60.83 & 5.89 \\
\hline 4952717 & 197.30 & 15.24 & & & & & & & & & & & \\
\hline 4991033 & 49.10 & 5.37 & 49.78 & 5.24 & 49.16 & 5.43 & 49.58 & 5.28 & & & 50.14 & 51.20 & 5.30 \\
\hline 4991732 & 104.70 & 8.49 & 104.85 & 8.54 & 104.68 & 8.44 & 104.41 & 8.54 & & & 105.28 & 104.84 & 8.50 \\
\hline 4995218 & 13.80 & 1.84 & & & & & 14.03 & 1.79 & & & & & \\
\hline 5000307 & 43.50 & 4.64 & 43.08 & 4.73 & 42.38 & 4.73 & 43.38 & 4.93 & 40.14 & 4.30 & 43.14 & 44.52 & 4.79 \\
\hline 5005217 & 37.50 & 4.27 & 39.58 & 4.34 & 39.29 & 4.30 & 37.96 & & 36.72 & & 40.32 & 42.22 & 4.31 \\
\hline 5006817 & 143.40 & 11.65 & 143.58 & 11.66 & 144.34 & 11.65 & 143.26 & 11.66 & 141.90 & 11.87 & 143.68 & 147.90 & 11.59 \\
\hline 5008996 & 30.40 & 3.74 & 30.65 & 3.73 & 30.25 & 3.75 & 30.58 & 3.70 & & & 30.86 & 32.15 & 3.73 \\
\hline 5009393 & 33.60 & 3.88 & 32.28 & 3.86 & & & 34.03 & & & & 33.09 & 34.44 & 4.02 \\
\hline 5016549 & 99.30 & 8.70 & 100.14 & 8.58 & 99.44 & 8.64 & 99.42 & 8.74 & & & 99.84 & 103.35 & 8.69 \\
\hline 5017711 & 36.30 & 4.17 & 33.77 & 4.17 & 33.96 & 4.58 & 37.16 & 4.11 & & & 35.58 & 36.76 & 4.20 \\
\hline 5023732 & & & 26.39 & 3.08 & & & & & & & & & \\
\hline 5023889 & & & 54.15 & 5.34 & & & & & & & & & \\
\hline 5023931 & & & 50.47 & 4.89 & & & & & & & & & \\
\hline 5023953 & & & 46.99 & 4.77 & & & & & & & & & \\
\hline 5024043 & & & 56.31 & 5.69 & & & & & & & & & \\
\hline 5024967 & & & 47.30 & 4.69 & & & & & & & & & \\
\hline 5026008 & 62.30 & 6.19 & 62.01 & 6.13 & 62.01 & 6.29 & 62.23 & 6.11 & 57.18 & 6.55 & 62.59 & 63.79 & 6.14 \\
\hline 5032140 & 47.80 & 4.68 & 47.69 & 4.64 & 47.63 & 4.78 & 47.91 & 4.66 & & & 47.53 & 47.77 & 4.69 \\
\hline 5034571 & 61.50 & 5.83 & 60.62 & 5.76 & 60.21 & 5.88 & 61.76 & 5.76 & & & 60.80 & 63.32 & 5.75 \\
\hline 5035849 & & & & & & & & & 30.86 & 3.27 & & & \\
\hline 5039392 & & & & & & & 6.61 & & & & & & \\
\hline 5084662 & 43.00 & 4.49 & 40.45 & 4.49 & 40.55 & 4.40 & 43.47 & 4.33 & & & 40.99 & 41.73 & 4.48 \\
\hline 5087542 & 35.60 & 3.80 & 35.80 & 3.77 & 34.54 & 3.71 & 35.16 & 3.76 & & & 35.86 & 35.45 & 3.83 \\
\hline 5088148 & 28.90 & 3.84 & 29.54 & 3.84 & & & 28.51 & 3.76 & & & 30.09 & 31.68 & 3.77 \\
\hline 5088362 & 39.40 & 4.12 & 39.37 & 4.16 & 38.87 & 4.12 & 39.44 & 4.07 & 37.27 & 4.41 & 39.71 & 40.77 & 4.12 \\
\hline 5090928 & & & & & & & & & 192.26 & 15.45 & & & \\
\hline 5091962 & 21.80 & 2.93 & 21.49 & 2.95 & 22.17 & 2.86 & 22.02 & 2.92 & & & 21.79 & 22.45 & \\
\hline 5095946 & 35.60 & 4.09 & 35.20 & 4.42 & 35.55 & 4.15 & 36.15 & 4.25 & 34.95 & 4.28 & 36.33 & 38.39 & 4.20 \\
\hline 5104573 & 35.10 & 3.93 & 36.23 & 4.41 & & & 35.49 & 3.80 & & & 36.65 & 38.90 & 3.90 \\
\hline 5104949 & 20.10 & 3.03 & 20.46 & 3.20 & 20.07 & 2.69 & 20.24 & 2.98 & & & 20.79 & 22.39 & 3.02 \\
\hline 5112288 & & & 45.67 & 4.90 & & & & & & & & & \\
\hline 5112361 & & & 67.03 & 6.17 & & & & & & & & & \\
\hline 5112373 & & & 44.25 & 4.60 & & & & & & & & & \\
\hline 5112387 & & & 44.68 & 4.80 & & & & & & & & & \\
\hline 5112730 & & & 44.47 & 5.62 & & & & & & & & & \\
\hline 5112744 & & & 41.93 & 4.38 & & & & & & & & & \\
\hline 5113041 & & & 37.75 & 3.97 & & & & & & & & & \\
\hline 5113061 & & & & & & & & & 12.28 & 1.70 & & & \\
\hline 5113910 & 2.40 & 0.59 & & & & & & & & & & & \\
\hline 5121116 & 21.60 & 2.61 & & & 19.74 & 2.60 & 21.53 & & 22.52 & 2.91 & 21.73 & 24.00 & 2.64 \\
\hline 5121605 & 33.20 & 4.09 & 32.92 & 4.14 & 32.58 & 4.20 & 32.81 & 4.07 & 35.24 & 4.17 & 33.29 & 34.48 & 4.10 \\
\hline 5128171 & 54.70 & 5.33 & 55.42 & 5.30 & 53.65 & 5.24 & 57.35 & 5.29 & 50.83 & 5.61 & 55.33 & 56.71 & 5.27 \\
\hline 5129011 & 40.90 & 4.68 & 40.21 & 4.53 & 41.38 & 4.74 & 41.37 & & & & 41.18 & 42.80 & 4.66 \\
\hline 5167473 & 30.70 & 4.00 & 30.86 & 4.35 & 30.86 & 3.96 & 30.93 & & & & 31.38 & 32.68 & 3.99 \\
\hline 5172229 & 39.90 & 4.38 & 39.59 & 4.43 & 39.75 & 4.43 & 39.94 & 4.44 & 37.83 & 4.36 & 39.98 & 40.94 & 4.36 \\
\hline 5174642 & & & & & 10.41 & 1.57 & 10.12 & & & & & & \\
\hline
\end{tabular}


Table 7. continued.

\begin{tabular}{|c|c|c|c|c|c|c|c|c|c|c|c|c|c|}
\hline Star & $\begin{array}{c}\text { COR } \\
v_{\max } \\
\mu \mathrm{Hz}\end{array}$ & $\begin{array}{c}\text { COR } \\
\Delta v \\
\mu \mathrm{Hz}\end{array}$ & $\begin{array}{l}\text { CAN } \\
v_{\max } \\
\mu \mathrm{Hz}\end{array}$ & $\begin{array}{c}\text { CAN } \\
\Delta v \\
\mu \mathrm{Hz}\end{array}$ & $\begin{array}{l}\mathrm{A} 2 \mathrm{Z} \\
v_{\max } \\
\mu \mathrm{Hz}\end{array}$ & $\begin{array}{c}\mathrm{A} 2 \mathrm{Z} \\
\Delta v \\
\mu \mathrm{Hz}\end{array}$ & $\begin{array}{l}\text { SYD } \\
v_{\max } \\
\mu \mathrm{Hz}\end{array}$ & $\begin{array}{c}\text { SYD } \\
\Delta v \\
\mu \mathrm{Hz}\end{array}$ & $\begin{array}{l}\text { DLB } \\
v_{\max } \\
\mu \mathrm{Hz}\end{array}$ & $\begin{array}{c}\text { DLB } \\
\Delta v \\
\mu \mathrm{Hz}\end{array}$ & $\begin{array}{c}\text { OCT I } \\
v_{\max } \\
\mu \mathrm{Hz}\end{array}$ & $\begin{array}{c}\text { OCT II } \\
v_{\max } \\
\mu \mathrm{Hz}\end{array}$ & $\begin{array}{c}\text { OCT } \\
\Delta v \\
\mu \mathrm{Hz}\end{array}$ \\
\hline 5184285 & & & & & & & & & 1.16 & 0.38 & & & \\
\hline 5196152 & 80.10 & 7.39 & 79.09 & 7.32 & 79.64 & 7.34 & 80.54 & 7.32 & & & 79.72 & 83.00 & 7.36 \\
\hline 5199906 & 68.10 & 5.83 & 69.33 & 6.14 & 68.98 & 5.81 & 67.30 & 5.85 & & & 69.92 & 72.94 & 5.86 \\
\hline 5266416 & 31.60 & 3.74 & & & 31.44 & 2.81 & 32.52 & 3.53 & 28.65 & 3.63 & 32.71 & 33.18 & 3.74 \\
\hline 5269165 & 29.90 & 3.74 & 29.18 & 3.74 & & & 31.37 & & & & 30.25 & 31.44 & 3.71 \\
\hline 5270737 & & & & & & & & & 1.07 & 0.37 & & & \\
\hline 5270933 & 36.70 & 4.14 & 36.78 & 4.27 & 36.61 & 4.15 & 36.92 & 4.22 & & & 36.99 & 37.85 & 4.17 \\
\hline 5272829 & 6.30 & 1.10 & & & & & & & & & & & \\
\hline 5283798 & 53.70 & 5.33 & 54.29 & 5.09 & 54.04 & 5.26 & 53.99 & & & & 55.09 & 56.38 & 5.27 \\
\hline 5284127 & 32.40 & 3.91 & 32.85 & 4.00 & 29.47 & 3.83 & 32.54 & 3.95 & & & 33.44 & 34.92 & 3.92 \\
\hline 5285357 & 32.80 & 4.04 & & & & & & & & & 33.03 & 33.75 & 3.95 \\
\hline 5287610 & 59.90 & 6.19 & 59.91 & 6.16 & 59.73 & 6.22 & 59.81 & 6.18 & 57.04 & 6.03 & 60.64 & 61.65 & 6.16 \\
\hline 5288607 & 55.50 & 5.73 & 57.00 & 6.23 & 58.11 & 5.71 & 53.49 & & 55.00 & & 58.75 & 60.39 & 5.70 \\
\hline 5288716 & 35.40 & 4.27 & & & 36.37 & 4.29 & 35.56 & 4.25 & & & 35.77 & 37.04 & 4.22 \\
\hline 5289959 & 6.50 & 1.12 & & & & & & & 5.74 & & & & \\
\hline 5297605 & 6.00 & 0.90 & & & & & & & & & & & \\
\hline 5307203 & 48.30 & 5.37 & 47.06 & 5.34 & 47.82 & 5.22 & 48.94 & 5.29 & 45.06 & 4.84 & 47.73 & 48.34 & 5.34 \\
\hline 5307747 & 85.40 & 6.87 & 84.27 & 7.06 & 85.82 & 6.92 & 85.36 & 6.99 & 81.06 & & 84.20 & 84.90 & 6.84 \\
\hline 5307930 & 50.10 & 4.46 & 48.87 & 4.87 & 51.86 & 4.52 & 50.03 & 4.55 & & & 53.51 & 53.61 & 4.59 \\
\hline 5339823 & 40.50 & 4.14 & 40.84 & 4.33 & 40.67 & 4.15 & 40.87 & 4.32 & 41.17 & & 41.14 & 44.27 & 4.22 \\
\hline 5353178 & & & 29.48 & 3.82 & & & 29.10 & & 30.82 & 4.07 & 29.69 & 31.36 & \\
\hline 5356201 & 202.90 & 15.62 & 210.10 & 15.81 & 210.00 & 15.36 & 209.08 & 15.88 & & & 208.23 & 202.59 & 15.76 \\
\hline 5360757 & 37.40 & 4.12 & 37.10 & 4.12 & 37.37 & 4.10 & 37.63 & 4.19 & & & 37.85 & 40.57 & 4.15 \\
\hline 5364240 & 71.50 & 6.81 & 72.26 & 6.76 & 71.46 & 6.75 & 71.28 & 6.78 & 73.10 & & 72.37 & 75.08 & 6.77 \\
\hline 5371626 & 21.40 & 2.59 & & & 21.48 & 2.67 & 21.60 & 2.53 & & & 21.63 & 22.31 & 2.56 \\
\hline 5380775 & 65.60 & 5.98 & 66.49 & 5.92 & 65.56 & 6.00 & 65.59 & 5.91 & & & 66.12 & 65.63 & 5.97 \\
\hline 5384584 & 15.10 & 2.10 & & & 16.30 & 2.17 & 15.42 & & 12.24 & 2.21 & & & \\
\hline 5385518 & 57.10 & 5.25 & 56.25 & 5.26 & 55.62 & 5.32 & 57.38 & 5.25 & 58.59 & 5.69 & 56.61 & 54.98 & 5.26 \\
\hline 5392657 & 45.20 & 4.55 & 44.79 & 4.60 & 44.97 & 4.49 & 45.58 & 4.55 & 40.06 & & 44.93 & 47.88 & 4.53 \\
\hline 5426041 & 50.40 & 5.05 & 51.23 & 5.09 & 50.04 & 5.10 & 50.33 & 5.07 & & & 51.03 & 53.42 & 5.02 \\
\hline 5430086 & & & & & & & 27.76 & 3.41 & & & & & \\
\hline 5430779 & 128.60 & 10.42 & 129.82 & 10.44 & 129.29 & 10.55 & 126.91 & 10.48 & & & 130.42 & 134.12 & 10.35 \\
\hline 5436082 & 39.40 & 4.38 & 38.78 & 4.38 & 38.84 & 4.38 & 39.71 & & & & 39.67 & 41.34 & 4.35 \\
\hline 5436814 & 9.70 & 1.68 & & & 9.67 & 1.17 & 10.04 & & 9.43 & & & & \\
\hline 5437909 & 199.40 & 14.94 & 205.93 & 14.55 & 200.00 & 15.48 & 204.74 & 15.29 & & & & & 15.19 \\
\hline 5449290 & & & & & & & & & 17.47 & 2.38 & & & \\
\hline 5457811 & 30.90 & 3.64 & 29.48 & 3.70 & 30.36 & 3.56 & 30.64 & 3.63 & 25.20 & & 31.44 & 33.54 & \\
\hline 5462797 & 12.10 & 1.54 & & & & & 12.13 & 1.59 & & & & & \\
\hline 5514079 & 86.80 & 7.90 & 88.39 & 7.82 & 88.60 & 7.88 & 87.10 & 7.85 & & & 88.19 & 91.53 & 7.92 \\
\hline 5514974 & 31.90 & 3.93 & 32.12 & 4.03 & 31.67 & 3.88 & 32.01 & 4.04 & 33.70 & 4.08 & 31.96 & 33.49 & 3.90 \\
\hline 5515314 & 137.00 & 10.91 & 136.10 & 10.83 & 137.58 & 10.76 & 137.10 & 10.86 & 137.09 & & 135.84 & 138.45 & 10.88 \\
\hline 5517118 & 32.10 & 3.93 & 31.72 & 3.89 & 31.57 & 3.90 & 32.36 & 3.86 & & & 31.89 & 33.22 & 3.95 \\
\hline 5517310 & 20.10 & 2.78 & 20.12 & 2.52 & 20.04 & 2.75 & 20.74 & & & & 20.33 & 21.04 & \\
\hline 5517442 & 34.60 & 3.97 & 34.90 & 3.97 & 35.20 & 3.95 & 35.41 & 3.97 & & & 35.49 & 36.60 & 3.94 \\
\hline 5524720 & 21.60 & 2.53 & 20.98 & 2.60 & 21.87 & & 21.70 & 2.62 & & & & & \\
\hline 5525572 & 27.70 & 3.37 & 28.37 & 3.39 & & & 27.41 & 3.87 & & & 28.72 & 30.40 & 4.12 \\
\hline 5526083 & 41.00 & 4.58 & 41.46 & 4.78 & 41.08 & 4.55 & 41.35 & 4.51 & & & 41.49 & 43.46 & 4.60 \\
\hline 5530598 & 103.00 & 8.70 & 100.52 & 8.61 & 102.90 & 8.71 & 104.39 & 8.68 & 103.00 & 8.55 & 103.78 & 107.35 & 8.66 \\
\hline 5546141 & 79.50 & 7.47 & 80.58 & 7.55 & 79.41 & 7.67 & 79.94 & 7.51 & 80.27 & 7.93 & 79.64 & 82.87 & 7.47 \\
\hline 5546749 & 36.60 & 3.97 & & & 35.43 & 4.00 & 36.52 & 4.04 & 41.83 & 4.43 & 37.71 & 38.53 & 4.01 \\
\hline 5553032 & 2.30 & 0.51 & & & & & & & & & & & \\
\hline 5597397 & 45.30 & 5.09 & 45.08 & 5.19 & 44.74 & 5.20 & 45.33 & 5.11 & & & 45.49 & 47.29 & 5.07 \\
\hline 5598645 & 87.90 & 6.55 & & & & & & & & & & & \\
\hline 5598789 & 56.20 & 5.46 & 56.86 & 5.41 & 56.24 & 5.48 & 55.47 & 5.43 & 53.91 & 5.45 & 57.12 & 58.35 & 5.39 \\
\hline 5599763 & 31.20 & 3.80 & 31.69 & 3.87 & 31.24 & 3.85 & 31.52 & 3.81 & & & 31.77 & 33.35 & 3.80 \\
\hline 5611192 & 99.10 & 7.99 & 99.97 & 7.95 & 99.48 & 7.76 & 97.91 & 8.07 & & & 101.14 & 105.88 & 7.91 \\
\hline 5612549 & 27.70 & 3.64 & & & 27.43 & 3.38 & & & 25.53 & 3.15 & 28.05 & 29.63 & 3.59 \\
\hline 5615439 & 14.70 & 2.41 & & & & & 14.67 & & & & & & \\
\hline 5617601 & & & & & & & & & 20.67 & 2.57 & & & \\
\hline 5631061 & 18.20 & 2.18 & & & & & 18.51 & & & & & & \\
\hline 5640750 & 24.30 & 2.83 & 24.02 & 2.95 & 24.10 & 2.86 & 24.65 & 2.98 & & & 24.39 & 24.57 & 2.81 \\
\hline 5648894 & 75.70 & 6.14 & 74.76 & 6.30 & 75.37 & 6.09 & 75.69 & 6.15 & 78.46 & & 75.23 & 78.30 & 6.14 \\
\hline 5685244 & 28.30 & 3.74 & & & & & 28.56 & 3.79 & & & & & \\
\hline 5685279 & 7.60 & 1.59 & & & 7.52 & 1.09 & 7.59 & & 8.21 & 1.39 & & & \\
\hline 5694720 & 31.00 & 3.35 & & & 30.82 & 3.41 & 30.63 & 3.35 & 29.34 & 3.77 & 31.53 & 33.64 & 3.39 \\
\hline
\end{tabular}


S. Hekker et al.: Comparison of global oscillation parameters from different methods

Table 7. continued.

\begin{tabular}{|c|c|c|c|c|c|c|c|c|c|c|c|c|c|}
\hline Star & $\begin{array}{c}\mathrm{COR} \\
v_{\max } \\
\mu \mathrm{Hz}\end{array}$ & $\begin{array}{c}\mathrm{COR} \\
\Delta v \\
\mu \mathrm{Hz}\end{array}$ & $\begin{array}{l}\text { CAN } \\
v_{\max } \\
\mu \mathrm{Hz}\end{array}$ & $\begin{array}{c}\text { CAN } \\
\Delta v \\
\mu \mathrm{Hz}\end{array}$ & $\begin{array}{l}\mathrm{A} 2 \mathrm{Z} \\
v_{\max } \\
\mu \mathrm{Hz}\end{array}$ & $\begin{array}{c}\mathrm{A} 2 \mathrm{Z} \\
\Delta v \\
\mu \mathrm{Hz}\end{array}$ & $\begin{array}{l}\text { SYD } \\
v_{\max } \\
\mu \mathrm{Hz}\end{array}$ & $\begin{array}{c}\text { SYD } \\
\Delta v \\
\mu \mathrm{Hz}\end{array}$ & $\begin{array}{l}\text { DLB } \\
v_{\max } \\
\mu \mathrm{Hz}\end{array}$ & $\begin{array}{c}\overline{\mathrm{DLB}} \\
\Delta v \\
\mu \mathrm{Hz}\end{array}$ & $\begin{array}{c}\text { OCT I } \\
v_{\max } \\
\mu \mathrm{Hz}\end{array}$ & $\begin{array}{c}\text { OCT II } \\
v_{\max } \\
\mu \mathrm{Hz}\end{array}$ & $\begin{array}{c}\mathrm{OCT} \\
\Delta v \\
\mu \mathrm{Hz}\end{array}$ \\
\hline 5698156 & 8.40 & 1.65 & & & & & 10.24 & & 8.67 & & & & \\
\hline 5700368 & 36.70 & 4.12 & 36.72 & 4.16 & & & 37.29 & 4.15 & 35.27 & 4.37 & 37.10 & 38.38 & 4.17 \\
\hline 5709564 & 26.40 & 3.84 & 26.32 & 3.75 & & & 26.68 & & 27.26 & 3.14 & 26.50 & 27.35 & \\
\hline 5709667 & 90.50 & 8.18 & 91.03 & 8.18 & 90.20 & 8.18 & 89.80 & 8.06 & & & 91.05 & 96.19 & 8.09 \\
\hline 5732847 & & & & & & & & & 27.24 & 3.35 & & & \\
\hline 5737655 & 31.30 & 4.32 & & & & & & & & & & & \\
\hline 5768953 & 10.10 & 1.66 & & & & & 10.82 & & & & & & \\
\hline 5770923 & 79.60 & 7.01 & 78.66 & & 79.21 & 6.92 & 78.75 & 6.99 & 68.45 & & 80.29 & 84.22 & 6.98 \\
\hline 5779724 & 5.90 & 1.13 & & & & & & & & & & & \\
\hline 5780782 & & & & & & & 11.11 & & 9.46 & & & & \\
\hline 5782127 & 26.80 & 3.58 & 26.48 & 3.59 & 26.23 & 3.25 & 26.85 & 3.45 & 24.14 & 3.13 & 27.27 & 28.78 & 3.54 \\
\hline 5782671 & 21.80 & 2.61 & 21.69 & 2.63 & 21.99 & 2.62 & 22.04 & 2.64 & 23.56 & 2.52 & 21.82 & 22.35 & 2.61 \\
\hline 5787509 & 31.40 & 3.86 & 31.82 & 3.84 & 31.50 & 3.92 & 31.28 & 3.82 & 30.65 & 3.83 & 32.22 & 33.46 & 3.85 \\
\hline 5790837 & 48.80 & 4.74 & 48.39 & 4.64 & 48.67 & 4.73 & 48.40 & 4.67 & 46.00 & 4.42 & 48.10 & 49.97 & 4.74 \\
\hline 5791135 & 42.30 & 4.43 & 43.47 & 4.43 & 42.85 & 4.59 & 42.31 & & & & 43.94 & 46.82 & 4.51 \\
\hline 5791844 & 51.20 & 5.17 & 51.84 & 5.32 & 52.26 & 5.14 & 53.18 & 5.21 & 46.69 & 5.34 & 53.28 & 55.59 & 5.14 \\
\hline 5793628 & 38.30 & 4.74 & & & & & 37.69 & & 35.09 & 4.32 & & & \\
\hline 5794100 & 34.30 & 4.17 & 34.22 & 4.28 & 34.13 & 4.13 & 34.39 & 4.12 & & & 34.60 & 35.72 & 4.23 \\
\hline 5795250 & 51.80 & 5.21 & 51.83 & 5.17 & 51.37 & 5.13 & 51.83 & 5.17 & & & 51.73 & 51.14 & 5.25 \\
\hline 5795626 & 39.90 & 4.55 & & & 39.52 & 4.52 & 40.08 & & & & 39.74 & 41.47 & 4.55 \\
\hline 5806522 & 60.50 & 5.98 & 58.49 & 5.85 & 59.47 & 5.88 & 60.89 & 5.92 & 56.01 & & 58.76 & 58.30 & 5.94 \\
\hline 5854149 & 41.70 & 4.35 & 41.19 & 4.31 & 40.77 & 4.30 & 41.50 & 4.36 & & & 41.61 & 42.51 & 4.36 \\
\hline 5855568 & 37.10 & 4.27 & 36.86 & 4.14 & 36.61 & 4.33 & 37.86 & & & & 37.48 & 38.89 & 4.22 \\
\hline 5856931 & 5.50 & 1.39 & & & & & & & 6.99 & & & & \\
\hline 5857618 & 71.40 & 6.31 & 70.06 & 6.33 & 70.76 & 6.29 & 71.33 & 6.31 & & & 70.99 & 72.35 & 6.25 \\
\hline 5857724 & 15.90 & 2.31 & 16.31 & 2.40 & 16.05 & 2.75 & 16.08 & 2.37 & & & 16.37 & 17.81 & 2.30 \\
\hline 5858034 & 42.10 & 4.38 & 40.87 & 4.45 & 40.72 & 4.27 & 42.54 & & & & 41.41 & 42.23 & 4.27 \\
\hline 5858947 & 165.30 & 14.63 & 165.94 & 14.99 & 165.21 & 14.49 & 164.22 & 14.51 & & & 166.40 & 168.00 & 14.48 \\
\hline 5859492 & 35.80 & 3.91 & 35.93 & 4.15 & 35.17 & 3.88 & 35.31 & & 34.44 & 4.22 & 36.24 & 37.83 & 3.95 \\
\hline 5866737 & 65.90 & 6.42 & & & 65.64 & 6.35 & 65.82 & 6.47 & 66.28 & & 66.35 & 66.53 & 6.44 \\
\hline 5866965 & 3.10 & 0.66 & & & & & & & & & & & \\
\hline 5872509 & 39.60 & 4.30 & 39.48 & 4.36 & 38.93 & 4.33 & 39.76 & 4.37 & 35.46 & 4.66 & 39.06 & 39.47 & \\
\hline 5887977 & 27.40 & 3.14 & 28.16 & 3.20 & 27.29 & 3.22 & 27.64 & 3.16 & 25.46 & & 28.32 & 29.73 & 3.13 \\
\hline 5891971 & 30.30 & 4.52 & & & & & 29.79 & & & & 31.72 & 33.48 & \\
\hline 5900096 & 31.00 & 3.88 & 31.52 & 3.97 & 31.03 & 3.86 & 30.93 & 3.89 & 29.22 & 3.83 & 31.76 & 32.94 & 3.88 \\
\hline 5903484 & 30.90 & 3.44 & 30.84 & 3.52 & 28.76 & 3.41 & 30.76 & 3.35 & & & 31.42 & 32.42 & 3.39 \\
\hline 5905655 & 13.80 & 2.76 & & & 14.89 & 1.81 & 14.42 & & & & & & \\
\hline 5940228 & 26.20 & 3.04 & 26.56 & 3.10 & 25.01 & 3.05 & 26.47 & 3.06 & & & 26.32 & 26.24 & 3.05 \\
\hline 5943345 & 47.90 & 4.68 & 49.97 & & 50.42 & 4.81 & 47.49 & 4.69 & 56.89 & & 51.52 & 55.13 & 4.70 \\
\hline 5950394 & 38.30 & 4.14 & 38.72 & 4.27 & 38.37 & 4.12 & 38.21 & 4.25 & 37.29 & & 39.23 & 40.66 & 4.16 \\
\hline 5950668 & 11.20 & 1.72 & & & & & 11.31 & & 11.93 & 1.78 & & & \\
\hline 5954370 & 15.50 & 2.40 & & & & & 16.33 & & & & & & \\
\hline 5955168 & & & & & & & & & 12.27 & 1.85 & & & \\
\hline 5959615 & 29.30 & 3.45 & 29.10 & 3.45 & 28.96 & 3.59 & 29.54 & 3.51 & & & 29.42 & 30.52 & \\
\hline 5963732 & 9.80 & 1.53 & & & & & 10.15 & & & & & & \\
\hline 5983299 & 64.80 & 5.83 & 59.93 & 5.76 & 61.19 & 5.88 & 63.88 & 5.80 & 55.65 & 5.96 & 62.18 & 63.22 & 5.90 \\
\hline 5990753 & & & 94.13 & & 99.36 & 7.60 & 97.64 & 7.56 & & & 100.12 & 105.96 & 7.69 \\
\hline 6029189 & 67.20 & 6.67 & 68.87 & 6.64 & 67.42 & 6.62 & 66.98 & 6.66 & 65.82 & 6.96 & 68.95 & 69.18 & 6.64 \\
\hline 6029474 & 75.30 & 7.09 & 75.62 & 7.17 & 75.02 & 7.27 & 75.35 & 7.17 & & & 75.74 & 76.90 & 7.14 \\
\hline 6032639 & & & & & 43.84 & 4.77 & 45.92 & & & & 45.13 & 46.89 & 4.87 \\
\hline 6033938 & 161.40 & 13.48 & 162.39 & 13.28 & 160.94 & 13.45 & 160.81 & 13.23 & & & 162.38 & 164.51 & 13.54 \\
\hline 6037660 & 30.80 & 4.09 & 30.71 & 4.09 & 29.86 & 4.17 & 30.93 & 4.10 & & & 30.77 & 31.82 & 4.01 \\
\hline 6037858 & 42.90 & 4.52 & 42.90 & 4.54 & & & 43.12 & 4.60 & 38.85 & & 43.17 & 45.76 & 4.54 \\
\hline 6043187 & 61.20 & 6.31 & 61.61 & 6.28 & 61.17 & 6.17 & 61.37 & 6.28 & & & 61.62 & 62.23 & 6.30 \\
\hline 6067958 & 16.30 & 2.32 & 17.02 & 2.50 & 16.80 & 2.31 & 16.38 & & & & 17.04 & 17.89 & 2.35 \\
\hline 6099979 & 40.00 & 4.49 & 40.61 & 4.59 & 40.03 & 4.52 & 40.23 & 4.63 & & & 40.61 & 40.83 & 4.50 \\
\hline 6101376 & 42.70 & 3.70 & & & & & & & & & & & \\
\hline 6103934 & 98.70 & 8.38 & 99.46 & 8.41 & 97.58 & 8.39 & 97.93 & 8.41 & 98.78 & & 98.92 & 102.82 & 8.39 \\
\hline 6104786 & & & & & 167.89 & 13.61 & 166.77 & 13.84 & & & 169.29 & 171.48 & 13.71 \\
\hline 6115575 & 50.60 & 4.91 & 50.69 & 4.85 & 49.97 & 4.87 & 50.55 & 4.86 & & & 50.70 & 50.17 & 4.85 \\
\hline 6117517 & 118.70 & 10.11 & 118.88 & 10.09 & 118.36 & 10.00 & 118.10 & 10.09 & 121.73 & & 118.74 & 122.51 & 10.12 \\
\hline 6118479 & 63.90 & 5.98 & 64.91 & 6.09 & 64.22 & 5.95 & 64.18 & 5.98 & 65.70 & & 64.96 & 63.70 & 5.93 \\
\hline 6123202 & 54.50 & 5.50 & 54.52 & 5.39 & 54.57 & 5.48 & 55.08 & 5.50 & 54.93 & & 54.96 & 54.83 & 5.42 \\
\hline 6125893 & 7.30 & 1.21 & & & & & 7.69 & & & & & & \\
\hline 6131884 & 26.30 & 3.66 & 26.14 & 3.70 & 26.23 & & 26.53 & 3.58 & & & 27.13 & 28.66 & \\
\hline
\end{tabular}


Table 7. continued.

\begin{tabular}{|c|c|c|c|c|c|c|c|c|c|c|c|c|c|}
\hline Star & $\begin{array}{l}\mathrm{COR} \\
v_{\max } \\
\mu \mathrm{Hz}\end{array}$ & $\begin{array}{c}\mathrm{COR} \\
\Delta v \\
\mu \mathrm{Hz}\end{array}$ & $\begin{array}{l}\text { CAN } \\
v_{\max } \\
\mu \mathrm{Hz}\end{array}$ & $\begin{array}{c}\text { CAN } \\
\Delta v \\
\mu \mathrm{Hz}\end{array}$ & $\begin{array}{l}\mathrm{A} 2 \mathrm{Z} \\
v_{\max } \\
\mu \mathrm{Hz}\end{array}$ & $\begin{array}{c}\mathrm{A} 2 \mathrm{Z} \\
\Delta v \\
\mu \mathrm{Hz}\end{array}$ & $\begin{array}{l}\text { SYD } \\
v_{\max } \\
\mu \mathrm{Hz}\end{array}$ & $\begin{array}{c}\text { SYD } \\
\Delta v \\
\mu \mathrm{Hz}\end{array}$ & $\begin{array}{c}\text { DLB } \\
v_{\max } \\
\mu \mathrm{Hz}\end{array}$ & $\begin{array}{c}\text { DLB } \\
\Delta v \\
\mu \mathrm{Hz}\end{array}$ & $\begin{array}{c}\text { OCT I } \\
v_{\max } \\
\mu \mathrm{Hz}\end{array}$ & $\begin{array}{c}\text { OCT II } \\
v_{\max } \\
\mu \mathrm{Hz}\end{array}$ & $\begin{array}{c}\mathrm{OCT} \\
\Delta v \\
\mu \mathrm{Hz}\end{array}$ \\
\hline 6140161 & 14.20 & 2.00 & 14.44 & 2.09 & & & 14.42 & 2.06 & 14.88 & 2.39 & & & \\
\hline 6144766 & 96.20 & 7.72 & & & & & & & & & & & \\
\hline 6144777 & 123.80 & 10.91 & 128.71 & 10.96 & 127.83 & 10.86 & 127.08 & 10.95 & 119.29 & & 128.53 & 132.25 & 10.83 \\
\hline 6150124 & 40.70 & 4.45 & & & & & & & & & & & \\
\hline 6185790 & 44.20 & 4.58 & 42.45 & 4.75 & 42.53 & 4.71 & 44.47 & 4.68 & & & 43.47 & 45.77 & 4.63 \\
\hline 6188269 & 64.60 & 5.63 & 62.93 & 5.73 & 61.31 & 5.67 & 64.38 & 5.70 & & & & & \\
\hline 6191288 & 25.70 & 3.10 & 25.83 & 3.17 & 25.54 & 3.08 & 26.01 & 3.20 & 24.07 & 3.14 & 25.83 & 26.87 & 3.13 \\
\hline 6192431 & 35.20 & 4.38 & 36.33 & 4.16 & 34.87 & 4.08 & 36.10 & 4.26 & 31.34 & 3.28 & 36.01 & 36.78 & 4.34 \\
\hline $\begin{array}{l}6196149 \\
6197019\end{array}$ & & & & & 26.92 & 3.36 & $\begin{array}{c}28.41 \\
9.90\end{array}$ & & & & & & \\
\hline 6198581 & 40.80 & 4.68 & 40.74 & 4.94 & 40.30 & 4.41 & 40.65 & & 39.43 & 4.64 & 41.15 & 44.46 & 4.48 \\
\hline 6200565 & 38.40 & 4.38 & 38.05 & 4.14 & & & 38.73 & & & & 38.02 & 37.56 & 4.18 \\
\hline 6231193 & 31.50 & 3.93 & 31.22 & 3.91 & 31.40 & 3.92 & 31.84 & 3.93 & 29.09 & 3.74 & 31.72 & 32.84 & 3.89 \\
\hline 6264398 & 29.30 & 3.26 & 30.47 & 3.26 & & & 30.93 & & & & 31.27 & 31.96 & \\
\hline 6276948 & 89.60 & 7.47 & 89.40 & 7.67 & 89.54 & 7.59 & 90.07 & 7.52 & 89.76 & 7.89 & 89.40 & 92.22 & 7.46 \\
\hline 6277741 & 24.40 & 2.93 & 24.74 & 3.06 & 24.42 & 3.01 & 24.60 & 3.03 & & & 24.75 & 25.78 & \\
\hline 6279491 & 28.50 & 3.88 & 28.03 & 3.72 & & & 29.08 & 3.71 & & & 28.62 & 29.97 & 3.05 \\
\hline 6279696 & & & & & & & & & 13.02 & 1.81 & & & \\
\hline 6286142 & 12.10 & 1.80 & 12.36 & 1.79 & 11.79 & & 12.27 & & & & & & \\
\hline 6304081 & 69.10 & 5.78 & 68.57 & 5.48 & 67.03 & 5.79 & 69.36 & 5.90 & 60.65 & 6.23 & 67.75 & 65.95 & 5.79 \\
\hline 6308068 & 6.30 & 1.14 & & & & & 6.18 & & 6.02 & 1.03 & & & \\
\hline 6345474 & 32.30 & 4.19 & 33.10 & 3.93 & & & 32.12 & & 34.81 & 4.65 & 33.19 & 35.07 & 4.18 \\
\hline 6357879 & 40.50 & 4.58 & 40.66 & 4.70 & 41.00 & 4.53 & 40.53 & 4.57 & & & 41.49 & 44.71 & 4.59 \\
\hline 6363090 & 44.20 & 4.61 & 42.59 & 4.64 & 42.25 & 4.64 & 45.47 & 4.53 & 35.59 & & 43.05 & 45.92 & 4.58 \\
\hline 6365867 & 27.90 & 3.72 & 28.01 & 3.85 & 27.86 & 3.56 & 27.83 & & & & 28.34 & 29.80 & 3.42 \\
\hline 6370549 & 10.00 & 1.82 & & & 9.73 & 1.28 & 10.32 & & & & & & \\
\hline 6371008 & 30.00 & 2.79 & 29.19 & 2.79 & & & 30.01 & & & & 29.81 & 32.71 & \\
\hline 6382830 & 21.80 & 2.46 & & & 20.87 & 2.50 & 22.72 & & 22.80 & 2.72 & & & \\
\hline 6418912 & 32.10 & 3.74 & & & & & 32.86 & 3.71 & & & 32.58 & 33.72 & 3.71 \\
\hline 6421579 & 8.20 & 1.49 & & & & & 8.12 & & & & & & \\
\hline 6424157 & 98.90 & 8.81 & 98.26 & 8.77 & 99.44 & 9.00 & 99.08 & 8.76 & & & 98.31 & 100.22 & 8.74 \\
\hline 6431941 & 20.90 & 2.89 & 20.74 & 2.91 & 21.31 & 2.88 & 21.06 & 2.86 & & & 21.07 & 22.08 & 2.89 \\
\hline 6444595 & 19.90 & 2.52 & 19.90 & 2.63 & & & 20.56 & & & & 20.01 & 20.16 & 2.52 \\
\hline 6448010 & 35.20 & 4.27 & 35.38 & 4.11 & 34.86 & 4.32 & 35.51 & 4.13 & & & 35.92 & 37.09 & 4.27 \\
\hline 6448764 & & & & & & & & & 2.11 & 0.57 & & & \\
\hline 6451238 & 29.90 & 3.86 & & & & & 30.19 & & & & & & \\
\hline 6462755 & 32.60 & 2.94 & 30.79 & 4.23 & & & 32.31 & & 30.16 & & 30.82 & 32.39 & \\
\hline 6465610 & 42.00 & 4.41 & 40.57 & 4.49 & 39.52 & 4.42 & 43.32 & 4.42 & 34.94 & 4.72 & 41.20 & 41.38 & 4.50 \\
\hline 6500038 & 52.40 & 5.37 & 52.77 & 5.42 & 51.57 & 5.37 & 52.53 & 5.40 & & & 52.24 & 50.44 & 5.32 \\
\hline 6500623 & 15.10 & 1.78 & & & 14.91 & 2.42 & 15.02 & & & & & & \\
\hline 6505025 & 32.00 & 4.19 & 32.66 & 4.42 & & & 31.74 & 4.22 & & & 33.20 & 35.16 & 4.20 \\
\hline 6508328 & 38.30 & 4.24 & 37.99 & 4.25 & 37.30 & 4.23 & 37.96 & 4.28 & & & 38.54 & 40.21 & 4.22 \\
\hline 6508943 & 32.30 & 4.12 & 32.83 & 4.12 & & & 31.38 & & & & & & \\
\hline 6522182 & 8.10 & 1.31 & & & & & 8.22 & & & & & & \\
\hline 6526377 & & & & & 33.78 & 3.67 & 33.09 & & & & & & \\
\hline 6543514 & 4.30 & 0.94 & & & & & & & & & & & \\
\hline $\begin{array}{l}6547007 \\
6549467\end{array}$ & 38.90 & 4.58 & & & 38.42 & 4.46 & $\begin{array}{c}39.35 \\
2.12\end{array}$ & 4.59 & 35.29 & & 38.70 & 39.55 & 4.54 \\
\hline 6579495 & 86.10 & 8.09 & 84.79 & 8.29 & 85.56 & 8.10 & 85.76 & 8.17 & & & 85.94 & 86.81 & 8.11 \\
\hline 6579998 & 31.80 & 4.32 & 32.22 & 4.21 & & & 32.41 & & & & 32.31 & 32.70 & 4.31 \\
\hline 6580479 & 34.30 & 4.17 & 34.22 & 4.10 & 33.37 & 4.17 & 34.09 & 4.16 & & & 35.63 & 37.71 & 4.15 \\
\hline 6586554 & 55.50 & 5.41 & 55.41 & 5.46 & 55.39 & 5.29 & 55.72 & 5.57 & & & 55.85 & 57.35 & 5.39 \\
\hline 6591336 & 23.40 & 2.99 & 23.96 & 2.99 & 23.91 & 3.03 & 23.73 & & & & & & \\
\hline 6593623 & 37.30 & 4.17 & 37.94 & 4.16 & 37.05 & 4.22 & 37.17 & 4.22 & & & 37.88 & 39.65 & 4.14 \\
\hline 6604237 & 3.40 & 0.74 & & & & & & & & & & & \\
\hline 6606106 & 31.80 & 4.07 & 31.72 & 4.16 & 31.61 & 3.34 & 32.17 & 3.98 & & & 32.18 & 33.09 & 4.06 \\
\hline 6610828 & 32.70 & 4.04 & 33.21 & 4.00 & 32.80 & 4.00 & 32.68 & & & & 33.48 & 34.72 & 4.07 \\
\hline 6612293 & 43.80 & 4.43 & & & 42.06 & 4.40 & 43.95 & 4.45 & 39.65 & 4.58 & 43.46 & 45.83 & 4.43 \\
\hline 6614588 & 39.90 & 4.35 & & & 40.91 & 4.38 & 40.14 & & 44.16 & 4.85 & 40.79 & 43.33 & 4.44 \\
\hline 6630797 & 2.90 & 0.58 & & & & & & & & & & & \\
\hline 6665058 & 106.40 & 10.11 & 104.04 & 9.69 & 106.31 & 10.00 & 106.55 & 9.69 & & & 104.93 & 106.21 & 9.79 \\
\hline 6680734 & 17.50 & 2.38 & 17.59 & 2.38 & 18.52 & 2.40 & 18.11 & 2.55 & & & 18.10 & 18.52 & 2.38 \\
\hline 6690139 & 114.60 & 9.68 & 115.26 & 9.70 & 114.58 & 9.80 & 114.07 & 9.68 & & & 115.45 & 119.24 & 9.69 \\
\hline 6696436 & 24.40 & 3.17 & 24.45 & 3.16 & 24.98 & 3.19 & 26.01 & 3.25 & & & 24.71 & 25.75 & 3.19 \\
\hline 6701238 & 28.50 & 4.00 & & & 28.92 & 4.15 & 29.07 & 4.05 & 26.57 & 3.51 & 29.14 & 31.47 & 4.11 \\
\hline
\end{tabular}


S. Hekker et al.: Comparison of global oscillation parameters from different methods

Table 7. continued.

\begin{tabular}{|c|c|c|c|c|c|c|c|c|c|c|c|c|c|}
\hline Star & $\begin{array}{l}\mathrm{COR} \\
v_{\max } \\
\mu \mathrm{Hz} \\
\end{array}$ & $\begin{array}{c}\text { COR } \\
\Delta v \\
\mu \mathrm{Hz}\end{array}$ & $\begin{array}{l}\text { CAN } \\
v_{\max } \\
\mu \mathrm{Hz}\end{array}$ & $\begin{array}{c}\text { CAN } \\
\Delta v \\
\mu \mathrm{Hz}\end{array}$ & $\begin{array}{l}\mathrm{A} 2 \mathrm{Z} \\
v_{\max } \\
\mu \mathrm{Hz}\end{array}$ & $\begin{array}{c}\mathrm{A} 2 \mathrm{Z} \\
\Delta v \\
\mu \mathrm{Hz}\end{array}$ & $\begin{array}{c}\text { SYD } \\
v_{\max } \\
\mu \mathrm{Hz} \\
\end{array}$ & $\begin{array}{c}\text { SYD } \\
\Delta v \\
\mu \mathrm{Hz}\end{array}$ & $\begin{array}{l}\text { DLB } \\
v_{\max } \\
\mu \mathrm{Hz}\end{array}$ & $\begin{array}{c}\mathrm{DLB} \\
\Delta v \\
\mu \mathrm{Hz} \\
\end{array}$ & $\begin{array}{c}\text { OCT I } \\
v_{\max } \\
\mu \mathrm{Hz}\end{array}$ & $\begin{array}{c}\text { OCT II } \\
v_{\max } \\
\mu \mathrm{Hz}\end{array}$ & $\begin{array}{c}\mathrm{OCT} \\
\Delta v \\
\mu \mathrm{Hz}\end{array}$ \\
\hline $\begin{array}{l}6718509 \\
6751876\end{array}$ & 28.80 & 4.00 & & & & & $\begin{array}{l}10.09 \\
30.14\end{array}$ & & & & 29.30 & 30.95 & 3.82 \\
\hline 6752023 & 86.20 & 6.37 & & & & & & & & & & & \\
\hline 6755907 & 43.70 & 4.88 & 43.75 & 4.82 & 43.65 & 4.88 & 44.00 & 4.84 & & & 43.97 & 45.41 & 4.82 \\
\hline 6762022 & 41.50 & 4.41 & 41.79 & 4.39 & 40.85 & 4.77 & 41.54 & 4.39 & & & 41.76 & 42.54 & 4.42 \\
\hline $\begin{array}{l}6765032 \\
6768319\end{array}$ & 52.70 & 5.54 & 53.91 & 5.60 & 53.10 & 5.61 & $\begin{array}{c}6.70 \\
52.55\end{array}$ & 5.57 & & & 54.16 & 56.64 & 5.52 \\
\hline 6779699 & 88.20 & 7.99 & 88.07 & 8.00 & 88.26 & 7.97 & 88.41 & 8.00 & 80.08 & & 87.41 & 90.50 & 8.00 \\
\hline 6780490 & 36.40 & 4.12 & & & 36.58 & 4.26 & 36.95 & & 35.46 & 4.14 & 37.26 & 38.37 & 4.25 \\
\hline 6785040 & 3.40 & 0.64 & & & & & & & & & & & \\
\hline 6804138 & 84.50 & 7.31 & 81.49 & 7.25 & 83.77 & 7.31 & 85.51 & 7.33 & & & 83.49 & 84.70 & 7.23 \\
\hline 6805631 & 42.80 & 4.64 & 42.49 & 4.50 & 42.42 & 4.57 & 43.20 & 4.52 & & & 42.68 & 44.09 & 4.59 \\
\hline 6837256 & 36.40 & 4.04 & 35.78 & 4.05 & 35.36 & 4.00 & 36.85 & 4.06 & 35.91 & 3.86 & 36.22 & 37.27 & 4.03 \\
\hline 6838420 & 28.70 & 3.45 & 29.22 & 3.62 & 28.25 & 3.55 & 28.23 & 3.49 & 26.98 & & 29.66 & 30.94 & 3.49 \\
\hline 6838707 & 33.10 & 4.04 & & & & & 33.10 & & 34.56 & 4.41 & & & \\
\hline 6843557 & 48.00 & 4.88 & 48.55 & 4.84 & 48.08 & 4.79 & 48.15 & 4.86 & 49.75 & & 49.46 & 51.11 & 4.90 \\
\hline 6849167 & 33.70 & 4.24 & & & 33.84 & 4.30 & 35.13 & & 25.62 & & & & \\
\hline 6851499 & 52.20 & 5.68 & 52.28 & 5.63 & 52.44 & 5.73 & 52.28 & 5.64 & & & 52.62 & 53.94 & 5.70 \\
\hline 6853465 & 85.90 & 7.55 & 86.61 & 7.46 & 85.46 & 7.41 & 84.80 & 7.51 & & & 87.15 & 90.38 & 7.56 \\
\hline 6853779 & 11.20 & 1.81 & & & & & 11.26 & & & & & & \\
\hline 6854095 & 30.70 & 4.00 & 30.33 & 4.00 & 30.52 & & 31.00 & & & & 31.12 & 31.78 & 4.08 \\
\hline 6860004 & & & & & & & 235.93 & 15.85 & & & & & \\
\hline 6878800 & 20.90 & 2.79 & 20.70 & 2.78 & 21.32 & 2.80 & 21.15 & 2.75 & & & 20.97 & 21.44 & \\
\hline 6888756 & 13.20 & 1.91 & & & & & 13.31 & 1.93 & & & 14.09 & 15.63 & 1.91 \\
\hline 6922899 & & & & & & & & & 3.35 & 0.71 & & & \\
\hline 6925158 & 89.40 & 7.90 & 90.44 & 7.99 & 89.37 & 7.88 & 89.28 & 8.04 & & & 90.26 & 94.18 & 7.93 \\
\hline 6928997 & 120.50 & 9.96 & 122.57 & 10.02 & 121.07 & 10.00 & 118.28 & 10.04 & & & 123.01 & 125.98 & 9.96 \\
\hline 6929729 & 65.40 & 6.08 & 66.20 & 6.04 & 65.10 & 6.08 & 65.15 & 6.07 & & & 66.21 & 67.92 & 6.05 \\
\hline 6936796 & 21.90 & 3.30 & & & 22.23 & 2.68 & 22.37 & & 20.51 & 2.24 & 22.89 & 24.36 & \\
\hline 6939158 & 30.90 & 3.35 & 31.36 & 3.35 & 30.54 & 3.33 & 31.00 & 3.46 & & & 31.11 & 29.88 & \\
\hline 6949816 & 79.20 & 7.99 & 80.39 & 8.00 & 79.58 & 7.95 & 78.84 & 7.92 & 78.50 & 8.43 & 80.48 & 82.32 & 8.08 \\
\hline 6951925 & 43.00 & 4.64 & 44.44 & 4.72 & 43.71 & 4.58 & 44.42 & 4.62 & 40.93 & 5.11 & 44.31 & 45.66 & 4.62 \\
\hline 6952140 & 8.60 & 1.35 & & & & & 8.69 & & 8.76 & 1.20 & & & \\
\hline 6952430 & 35.80 & 4.24 & 36.26 & 4.42 & 36.24 & 4.17 & 35.06 & 4.16 & & & 36.55 & 38.08 & 4.23 \\
\hline 6964342 & 41.40 & 4.49 & 41.52 & 4.38 & 40.09 & 4.49 & 41.79 & 4.40 & 42.48 & & 41.15 & 43.26 & 4.49 \\
\hline 7006979 & 35.10 & 4.07 & 35.63 & 3.73 & 35.40 & 4.05 & 35.48 & 4.20 & 35.48 & 4.34 & 35.62 & 37.39 & 4.01 \\
\hline 7018252 & 26.50 & 3.37 & & & & & 26.37 & & 27.69 & 3.52 & 26.98 & 27.77 & 3.34 \\
\hline 7020371 & 15.50 & 2.12 & 15.43 & 2.12 & & & 15.56 & & & & 15.49 & 16.02 & 2.09 \\
\hline 7024018 & 84.50 & 6.67 & 81.80 & 6.90 & 80.20 & 6.86 & 85.61 & 6.70 & & & 86.90 & 90.83 & 6.81 \\
\hline 7031016 & 33.40 & 4.17 & 33.75 & 4.09 & 33.56 & 4.17 & 33.92 & 4.17 & & & 34.10 & 35.61 & 4.15 \\
\hline 7039075 & 45.10 & 4.74 & 45.10 & 4.79 & 45.03 & 4.82 & 44.73 & 4.76 & 45.24 & 5.24 & 46.01 & 48.33 & 4.72 \\
\hline 7044886 & 43.50 & 4.17 & 42.15 & 4.22 & 42.33 & 4.20 & 43.90 & 4.16 & & & 43.58 & 43.27 & 4.15 \\
\hline 7048889 & 29.60 & 3.78 & 29.39 & 3.75 & 29.34 & 3.83 & 30.48 & 3.76 & & & 30.04 & 31.66 & 3.82 \\
\hline 7049306 & 32.60 & 4.07 & 33.35 & 4.32 & 32.40 & 3.94 & 32.89 & 3.93 & & & 33.50 & 34.64 & 4.12 \\
\hline 7060732 & 136.00 & 10.91 & 133.56 & 10.84 & 132.33 & 10.91 & 132.25 & 10.86 & & & 133.18 & 136.24 & 10.90 \\
\hline 7092067 & 51.30 & 5.46 & 51.69 & 5.46 & & & 51.42 & 5.49 & & & 51.74 & 52.78 & 5.46 \\
\hline 7093179 & 31.10 & 3.70 & 30.66 & 3.71 & 29.59 & 3.62 & 31.52 & 3.73 & & & 30.77 & 31.01 & 3.64 \\
\hline 7098252 & 24.80 & 3.30 & 25.28 & 3.03 & & & 25.46 & & & & 24.57 & 24.44 & \\
\hline 7098412 & & & & & & & 6.93 & & & & & & \\
\hline 7100768 & & & & & 23.79 & 2.38 & 24.47 & & & & & & \\
\hline 7100955 & & & & & & & 4.83 & & & & & & \\
\hline 7103297 & 40.50 & 4.41 & 39.73 & 4.41 & 38.43 & 4.33 & 40.46 & 4.35 & & & 39.92 & 39.84 & 4.40 \\
\hline 7109120 & 61.90 & 5.93 & 61.56 & 6.01 & 61.50 & 5.85 & 62.65 & 5.98 & 62.43 & & 62.09 & 62.63 & 5.92 \\
\hline 7118721 & 69.20 & 6.08 & 67.62 & 6.11 & 68.05 & 6.03 & 70.21 & 6.11 & & & 68.22 & 69.26 & 6.18 \\
\hline 7134640 & & & & & & & & & 4.34 & 1.00 & & & \\
\hline 7177015 & 82.80 & 7.47 & 84.20 & 7.48 & 82.87 & 7.34 & 82.61 & 7.46 & 74.71 & 7.67 & 84.43 & 87.30 & 7.48 \\
\hline 7204369 & 16.50 & 2.15 & 16.38 & 2.14 & & & 16.50 & & 15.53 & 2.11 & 16.47 & 16.96 & \\
\hline 7205067 & 60.00 & 5.29 & & & 61.47 & 5.26 & & & 60.02 & & 62.37 & 66.96 & 5.40 \\
\hline 7206531 & 44.90 & 4.71 & 46.32 & 4.71 & 45.57 & 4.65 & 44.93 & & & & 46.25 & 47.76 & 4.64 \\
\hline 7213716 & 15.40 & 1.93 & & & & & 15.34 & & & & 15.09 & 15.52 & 1.93 \\
\hline 7258771 & 83.10 & 7.47 & 83.04 & 7.77 & 83.20 & 7.59 & 83.21 & 7.45 & & & 84.13 & 83.48 & 7.52 \\
\hline 7259653 & 38.30 & 4.46 & 39.01 & 4.39 & 39.68 & 4.33 & 37.68 & 4.42 & & & 40.44 & 41.31 & 4.39 \\
\hline 7270142 & 52.20 & 5.46 & 53.19 & 5.44 & 52.21 & 5.45 & 52.34 & 5.45 & & & 52.83 & 54.69 & 5.45 \\
\hline 7272257 & 23.90 & 3.06 & 23.70 & 3.04 & 23.28 & & 24.08 & 3.03 & & & 23.92 & 24.43 & 3.01 \\
\hline 7273672 & 20.90 & 2.78 & 21.07 & 2.67 & 20.87 & 2.18 & 21.15 & & 21.88 & 2.90 & 21.18 & 21.88 & 2.81 \\
\hline
\end{tabular}


Table 7. continued.

\begin{tabular}{|c|c|c|c|c|c|c|c|c|c|c|c|c|c|}
\hline Star & $\begin{array}{l}\mathrm{COR} \\
v_{\max } \\
\mu \mathrm{Hz}\end{array}$ & $\begin{array}{c}\mathrm{COR} \\
\Delta v \\
\mu \mathrm{Hz}\end{array}$ & $\begin{array}{l}\text { CAN } \\
v_{\max } \\
\mu \mathrm{Hz}\end{array}$ & $\begin{array}{c}\text { CAN } \\
\Delta v \\
\mu \mathrm{Hz}\end{array}$ & $\begin{array}{l}\mathrm{A} 2 \mathrm{Z} \\
v_{\max } \\
\mu \mathrm{Hz}\end{array}$ & $\begin{array}{c}\mathrm{A} 2 \mathrm{Z} \\
\Delta v \\
\mu \mathrm{Hz}\end{array}$ & $\begin{array}{l}\text { SYD } \\
v_{\max } \\
\mu \mathrm{Hz}\end{array}$ & $\begin{array}{c}\text { SYD } \\
\Delta v \\
\mu \mathrm{Hz}\end{array}$ & $\begin{array}{c}\text { DLB } \\
v_{\max } \\
\mu \mathrm{Hz}\end{array}$ & $\begin{array}{c}\text { DLB } \\
\Delta v \\
\mu \mathrm{Hz}\end{array}$ & $\begin{array}{c}\text { OCT I } \\
v_{\max } \\
\mu \mathrm{Hz}\end{array}$ & $\begin{array}{c}\text { OCT II } \\
v_{\max } \\
\mu \mathrm{Hz}\end{array}$ & $\begin{array}{c}\mathrm{OCT} \\
\Delta v \\
\mu \mathrm{Hz}\end{array}$ \\
\hline 7281777 & 38.10 & 4.95 & 36.83 & 4.95 & & & & & & & 38.41 & 39.33 & 5.05 \\
\hline 7289277 & 29.20 & 3.78 & 29.35 & 3.84 & 29.07 & 3.77 & 29.55 & & & & 29.22 & 31.46 & 3.75 \\
\hline 7293531 & 17.10 & 2.72 & & & 17.81 & 2.66 & 21.06 & & 16.80 & 2.80 & & & \\
\hline 7293609 & 30.40 & 3.76 & 31.01 & 3.74 & & & 31.05 & & & & 31.27 & 33.22 & 3.72 \\
\hline 7294715 & 42.20 & 4.55 & 42.01 & 4.89 & 42.32 & 3.98 & 43.03 & & & & 42.32 & 43.86 & 4.48 \\
\hline 7297940 & 100.30 & 8.19 & 99.76 & 8.14 & 99.75 & 8.13 & 99.90 & 8.13 & & & 100.00 & 103.82 & 8.16 \\
\hline 7303187 & 24.40 & 3.21 & 24.59 & 3.41 & & & 24.64 & & & & 25.56 & 27.54 & 3.27 \\
\hline 7337994 & 28.60 & 4.09 & & & & & 29.14 & 4.27 & 24.19 & 2.91 & 29.17 & 29.92 & 4.01 \\
\hline 7340724 & 128.40 & 10.74 & 128.80 & 10.61 & 129.46 & 10.66 & 127.98 & 10.59 & & & 128.79 & 131.09 & 10.57 \\
\hline 7341343 & 14.10 & 1.98 & 13.88 & 1.91 & & & 14.37 & & 14.40 & 2.32 & & & \\
\hline 7346365 & 53.00 & 5.33 & 53.20 & 5.39 & 52.71 & 5.40 & 53.08 & 5.32 & & & 53.68 & 54.41 & 5.35 \\
\hline 7346442 & 48.80 & 5.17 & 48.41 & 5.21 & & & 49.38 & 5.22 & & & 48.55 & 50.31 & 5.17 \\
\hline 7365701 & 31.60 & 4.14 & 31.54 & 4.19 & & & 31.42 & & & & 31.95 & 33.41 & 3.96 \\
\hline 7366121 & 43.70 & 4.43 & 43.39 & 4.65 & 43.56 & 4.31 & 43.47 & & & & 43.91 & 45.93 & 4.37 \\
\hline 7366820 & & & & & 35.91 & 4.20 & 38.18 & & & & 37.10 & 36.04 & 4.09 \\
\hline 7374855 & 36.30 & 3.68 & 39.50 & 3.68 & 37.47 & 3.57 & 37.66 & 3.68 & & & 39.49 & 40.52 & 3.65 \\
\hline 7378357 & 7.30 & 1.29 & & & & & 7.32 & & 7.76 & & & & \\
\hline 7382407 & 43.50 & 4.77 & 43.29 & 4.67 & 43.49 & 4.79 & 43.89 & 4.74 & 42.47 & & 43.54 & 44.65 & 4.65 \\
\hline 7384523 & 33.70 & 4.12 & 33.27 & 4.12 & & & 33.50 & 4.15 & & & 34.50 & 36.23 & 4.13 \\
\hline 7387188 & 32.60 & 4.14 & 33.39 & 4.14 & & & 32.96 & & & & 33.51 & 35.18 & 4.12 \\
\hline 7420223 & 34.60 & 4.22 & 35.52 & 4.30 & 34.31 & 4.01 & 34.96 & 4.28 & 34.61 & 3.99 & 35.70 & 38.06 & 4.20 \\
\hline 7429055 & 58.00 & 6.08 & & & 59.56 & 6.15 & 58.84 & 6.11 & 62.01 & 5.85 & 60.70 & 62.91 & 6.09 \\
\hline 7435143 & 59.50 & 6.19 & 60.34 & 6.19 & 60.80 & 6.22 & 59.97 & 6.20 & & & 62.08 & 61.17 & 6.21 \\
\hline 7439931 & & & & & 258.76 & 17.12 & 241.55 & & & & & & \\
\hline 7445698 & 41.40 & 4.17 & 41.13 & 4.13 & 39.64 & 4.04 & 41.57 & 4.18 & & & 41.14 & 40.58 & 4.14 \\
\hline 7446560 & 12.30 & 1.98 & & & & & 12.45 & & & & & & \\
\hline 7448051 & 34.10 & 3.78 & 33.90 & 3.83 & 33.95 & 3.85 & 34.27 & 3.89 & 34.50 & 3.41 & 33.79 & 33.97 & 3.79 \\
\hline 7448746 & 4.90 & 0.91 & & & & & & & & & & & \\
\hline 7457184 & 67.30 & 5.88 & 67.53 & 5.99 & 64.96 & 5.93 & 65.45 & 5.93 & & & 68.06 & 70.74 & 5.86 \\
\hline 7466616 & 76.40 & 6.19 & 72.69 & & 75.08 & 6.17 & 75.29 & 6.32 & & & 74.80 & 77.28 & 6.21 \\
\hline 7500388 & 39.50 & 4.91 & 39.42 & 4.67 & 39.15 & 4.65 & 39.54 & 4.71 & & & 39.89 & 40.98 & \\
\hline 7513379 & 105.60 & 9.05 & 108.85 & 9.01 & 109.67 & 9.04 & 107.71 & 9.10 & & & 109.52 & 112.61 & 9.02 \\
\hline 7522297 & 32.80 & 4.19 & 31.95 & 4.15 & 32.27 & 4.09 & 33.08 & 4.14 & & & 33.28 & 35.01 & 4.16 \\
\hline 7523875 & 85.30 & 7.72 & 85.91 & 7.68 & 84.73 & 7.65 & 84.05 & 7.69 & 89.07 & & 85.97 & 91.24 & 7.68 \\
\hline 7529215 & 90.40 & 8.49 & 90.33 & 8.30 & 90.37 & 8.33 & 90.69 & 8.30 & & & 90.24 & 92.14 & 8.47 \\
\hline 7540130 & 12.80 & 1.67 & & & & & 12.87 & & 12.38 & 1.93 & & & \\
\hline 7543672 & 19.10 & 2.15 & & & 18.57 & 2.14 & 19.28 & 2.21 & & & 19.26 & 19.91 & 2.15 \\
\hline 7549472 & & & & & & & 4.21 & & 3.18 & 0.74 & & & \\
\hline 7552779 & & & & & & & 7.59 & & 5.16 & & & & \\
\hline 7581399 & 86.20 & 6.55 & & & & & & & & & & & \\
\hline 7581822 & 161.50 & 13.22 & 167.24 & 13.03 & 161.27 & 13.14 & 161.57 & 13.08 & & & 166.51 & 171.06 & 13.15 \\
\hline 7584122 & & & & & 239.87 & 18.22 & 250.05 & & & & & & 18.06 \\
\hline 7585523 & 37.70 & 3.93 & 35.96 & 3.98 & 35.31 & 3.93 & 37.96 & 3.94 & & & 36.99 & 38.01 & 3.91 \\
\hline 7587099 & 36.90 & 3.76 & & & 35.75 & 3.95 & 37.95 & & & & 36.43 & 37.10 & 3.81 \\
\hline 7591642 & 25.10 & 3.58 & & & & & 24.52 & & & & & & \\
\hline 7594865 & 30.10 & 3.91 & 30.24 & 4.22 & 29.70 & 3.81 & 30.36 & 3.93 & & & 30.41 & 31.51 & 3.92 \\
\hline 7595155 & 26.50 & 3.60 & 27.07 & 3.60 & & & 25.98 & & & & & & \\
\hline 7595285 & 29.40 & 3.95 & & & 27.42 & 4.33 & 29.49 & & 29.86 & 3.41 & & & \\
\hline 7596701 & 45.50 & 5.09 & 45.64 & 5.08 & 43.83 & 5.00 & 45.46 & 5.13 & & & 45.65 & 47.14 & 5.03 \\
\hline 7611069 & 154.40 & 12.50 & 156.58 & 12.56 & 155.72 & 12.35 & 154.22 & 12.63 & & & 156.77 & 157.87 & 12.57 \\
\hline 7618122 & 71.20 & 6.94 & 70.68 & 6.97 & 70.60 & 6.86 & 71.53 & 7.05 & & & 70.93 & 70.80 & 6.91 \\
\hline 7619034 & 44.30 & 4.77 & 44.25 & 4.85 & 44.27 & 4.76 & 45.05 & & 43.92 & 4.85 & 44.90 & 47.43 & 4.65 \\
\hline 7619745 & & & & & 167.25 & 12.99 & 165.31 & 13.12 & & & 168.56 & 178.67 & 12.98 \\
\hline 7626457 & 176.20 & 13.22 & 176.24 & 13.32 & 176.07 & & 174.96 & 13.33 & & & 175.52 & 175.33 & 13.29 \\
\hline 7661609 & 21.40 & 2.70 & 21.20 & 2.69 & 18.89 & 2.39 & 21.52 & 2.73 & & & 21.66 & 22.36 & 2.68 \\
\hline 7662025 & 28.70 & 4.09 & 29.54 & 3.79 & 28.36 & 3.80 & 29.39 & & & & 29.98 & 31.70 & 4.18 \\
\hline 7663241 & 25.00 & 2.86 & 24.77 & 3.02 & 23.76 & 3.00 & 25.19 & & & & & & \\
\hline 7667657 & 44.80 & 4.44 & 43.33 & 4.64 & 43.05 & 4.45 & 44.86 & & & & 44.47 & 44.99 & 4.44 \\
\hline 7668782 & 14.50 & 2.06 & & & 14.86 & 2.05 & 14.60 & 2.04 & & & & & \\
\hline 7670419 & 29.50 & 3.72 & & & & & 30.59 & & 26.08 & 3.62 & & & \\
\hline 7671562 & 30.80 & 3.76 & & & 29.61 & 3.75 & 30.81 & 3.77 & & & 31.43 & 32.35 & 3.78 \\
\hline 7672405 & 12.30 & 1.76 & & & & & 13.22 & & & & & & \\
\hline 7674224 & & & & & 131.40 & 10.71 & 130.94 & 10.70 & & & 130.84 & 134.94 & 10.72 \\
\hline 7677317 & 22.10 & 3.08 & 23.54 & 3.14 & 21.34 & 3.07 & 21.89 & 3.05 & 22.66 & 2.66 & 24.00 & 25.36 & 3.08 \\
\hline 7685724 & 36.30 & 4.12 & 34.84 & 4.12 & 35.16 & 4.19 & 36.33 & & & & 35.63 & 37.07 & 4.16 \\
\hline
\end{tabular}


S. Hekker et al.: Comparison of global oscillation parameters from different methods

Table 7. continued.

\begin{tabular}{|c|c|c|c|c|c|c|c|c|c|c|c|c|c|}
\hline Star & $\begin{array}{l}\mathrm{COR} \\
\nu_{\max } \\
\mu \mathrm{Hz}\end{array}$ & $\begin{array}{c}\text { COR } \\
\Delta v \\
\mu \mathrm{Hz}\end{array}$ & $\begin{array}{l}\text { CAN } \\
\nu_{\max } \\
\mu \mathrm{Hz}\end{array}$ & $\begin{array}{c}\text { CAN } \\
\Delta v \\
\mu \mathrm{Hz}\end{array}$ & $\begin{array}{l}\mathrm{A} 2 \mathrm{Z} \\
\nu_{\max } \\
\mu \mathrm{Hz}\end{array}$ & $\begin{array}{c}\mathrm{A} 2 \mathrm{Z} \\
\Delta v \\
\mu \mathrm{Hz}\end{array}$ & $\begin{array}{l}\text { SYD } \\
v_{\max } \\
\mu \mathrm{Hz}\end{array}$ & $\begin{array}{c}\text { SYD } \\
\Delta v \\
\mu \mathrm{Hz}\end{array}$ & $\begin{array}{c}\text { DLB } \\
v_{\max } \\
\mu \mathrm{Hz}\end{array}$ & $\begin{array}{c}\text { DLB } \\
\Delta v \\
\mu \mathrm{Hz}\end{array}$ & $\begin{array}{c}\text { OCT I } \\
v_{\max } \\
\mu \mathrm{Hz}\end{array}$ & $\begin{array}{c}\text { OCT II } \\
v_{\max } \\
\mu \mathrm{Hz}\end{array}$ & $\begin{array}{c}\mathrm{OCT} \\
\Delta v \\
\mu \mathrm{Hz}\end{array}$ \\
\hline 7693833 & 31.30 & 4.19 & 31.76 & 4.01 & 31.36 & 3.86 & 31.48 & 4.05 & & & 32.17 & 33.84 & 4.13 \\
\hline 7697146 & 61.30 & 5.88 & 61.23 & 5.79 & 61.31 & 5.89 & 61.71 & 5.80 & 62.65 & 5.60 & 61.90 & 62.98 & 5.85 \\
\hline 7701048 & 42.10 & 4.14 & 42.22 & 4.20 & 42.11 & 4.13 & 42.34 & 4.18 & 44.59 & & 42.10 & 40.82 & 4.10 \\
\hline 7729396 & 8.30 & 1.41 & & & & & 8.76 & 1.39 & 9.35 & 1.65 & & & \\
\hline 7732065 & 12.50 & 1.91 & & & & & 12.64 & 1.88 & & & & & \\
\hline 7732335 & 81.80 & 7.64 & 79.38 & 7.69 & 81.14 & 7.59 & 81.72 & 7.65 & 73.32 & 7.70 & 82.02 & 83.69 & 7.67 \\
\hline 7734065 & 26.30 & 3.60 & 26.57 & 3.20 & & & 26.53 & & & & 26.92 & 28.33 & 3.57 \\
\hline 7749249 & 71.70 & 6.67 & 70.48 & 6.62 & 69.28 & 6.76 & 71.11 & 6.67 & & & 70.68 & 71.89 & 6.64 \\
\hline 7764335 & & & & & & & 9.77 & & & & & & \\
\hline 7771603 & 34.20 & 3.74 & 35.17 & 3.88 & & & 35.02 & 3.75 & & & & & \\
\hline 7798339 & & & 29.57 & 3.86 & & & & & & & & & \\
\hline 7799762 & 17.70 & 2.25 & 16.97 & 2.25 & 15.30 & 2.19 & 18.78 & 2.23 & & & 17.93 & 18.34 & 2.27 \\
\hline 7802551 & 13.60 & 1.82 & & & 13.18 & 1.85 & 13.61 & 1.75 & & & & & \\
\hline 7808227 & 31.60 & 4.30 & 32.67 & 4.24 & & & 31.85 & & & & 32.77 & 34.08 & \\
\hline 7820638 & 48.70 & 4.85 & & & & & & & & & & & \\
\hline 7821234 & 33.70 & 4.14 & 34.51 & 4.11 & & & 34.79 & 4.16 & & & 34.65 & 36.88 & 4.10 \\
\hline 7831348 & 37.50 & 4.04 & 36.68 & 4.10 & 37.55 & 3.98 & 38.15 & 4.11 & & & 37.06 & 37.87 & 4.13 \\
\hline 7841410 & 7.10 & 1.32 & & & & & 7.25 & & & & & & \\
\hline 7867854 & 31.20 & 4.24 & 30.18 & 4.24 & & & 29.76 & & & & 31.93 & 32.85 & \\
\hline 7868532 & 72.40 & 7.09 & 73.37 & 7.10 & 72.48 & 7.22 & 72.32 & 7.09 & & & 74.13 & 76.67 & 7.06 \\
\hline 7872519 & 32.70 & 4.32 & 33.07 & 4.13 & & & 32.46 & 4.01 & & & 33.33 & 34.33 & \\
\hline 7888403 & 47.80 & 5.25 & 48.26 & 5.28 & 47.59 & 5.21 & 46.92 & 5.26 & & & 48.27 & 50.08 & 5.27 \\
\hline 7905696 & 45.20 & 4.64 & 45.74 & 4.57 & 44.51 & 4.69 & 45.23 & 4.56 & & & 45.81 & 47.34 & 4.60 \\
\hline 7909976 & 76.00 & 6.12 & & & & & & & & & & & \\
\hline 7919558 & 39.20 & 4.04 & 37.85 & 4.04 & 38.68 & 4.05 & 39.29 & 4.03 & & & 38.79 & 39.74 & 4.01 \\
\hline 7935931 & & & & & & & 30.88 & & & & & & \\
\hline 7936033 & 14.70 & 2.06 & & & 14.79 & 2.11 & 14.90 & 2.07 & & & & & \\
\hline 7938029 & 20.40 & 2.77 & 21.22 & 2.73 & 20.27 & 2.72 & 20.51 & 2.74 & & & & & \\
\hline 7940959 & 6.40 & 1.20 & & & & & & & & & & & \\
\hline 7944142 & 75.60 & 7.09 & 75.03 & 7.04 & 75.34 & 7.10 & 75.54 & 7.03 & & & 75.71 & 76.59 & 7.03 \\
\hline 7948086 & 41.20 & 4.55 & 41.72 & 4.50 & 41.91 & 4.59 & 41.28 & 4.53 & 40.17 & 4.67 & 42.15 & 45.02 & 4.53 \\
\hline 7949599 & 32.80 & 3.93 & 32.84 & 4.17 & 32.55 & 3.92 & 33.28 & 4.13 & 35.37 & 4.55 & 33.16 & 33.96 & 3.93 \\
\hline 7950229 & 42.60 & 3.86 & & & & & & & & & & & \\
\hline 7955392 & 42.30 & 4.09 & 43.57 & 4.14 & 42.94 & 4.18 & 41.97 & 4.20 & & & 44.03 & 46.31 & 4.14 \\
\hline 7957166 & & & & & 205.83 & 14.67 & 197.99 & 14.34 & & & & & \\
\hline 7966761 & & & & & & & & & 18.53 & 2.76 & & & \\
\hline 7967534 & 29.40 & 4.09 & 30.16 & 4.10 & & & 30.26 & 4.07 & & & & & \\
\hline 7973937 & 2.90 & 0.62 & & & & & & & & & & & \\
\hline 7974439 & & & & & & & 8.95 & & & & & & \\
\hline 7989213 & 8.40 & 1.42 & & & & & 8.44 & & & & & & \\
\hline 8005116 & 37.70 & 4.19 & 36.82 & 4.31 & 36.76 & 4.24 & 37.76 & 4.28 & & & 37.59 & 39.06 & 4.09 \\
\hline 8005874 & 9.70 & 1.57 & & & & & 9.90 & 1.57 & 9.63 & & & & \\
\hline 8008492 & & & & & & & & & 39.41 & 3.52 & & & \\
\hline 8017159 & 3.20 & 0.60 & & & & & & & & & & & \\
\hline 8037095 & & & & & & & & & 2.27 & 0.62 & & & \\
\hline 8039416 & 31.80 & 4.00 & 31.57 & 4.12 & 31.28 & 3.98 & 32.04 & 4.02 & & & 32.26 & 33.96 & 3.98 \\
\hline 8041612 & 3.80 & 0.67 & & & & & 3.78 & & & & & & \\
\hline 8045883 & 30.90 & 4.00 & 29.82 & 4.03 & 29.87 & 3.86 & 31.16 & 4.04 & 26.11 & 3.53 & 30.93 & 31.56 & 3.83 \\
\hline 8056874 & 52.50 & 5.29 & 51.82 & 5.31 & 52.65 & 5.38 & 51.29 & 5.24 & & & 52.97 & 57.94 & 5.20 \\
\hline 8078554 & 34.20 & 4.22 & 35.23 & 4.24 & 34.53 & 4.22 & 34.52 & 4.16 & 36.80 & & 35.34 & 36.92 & 4.15 \\
\hline 8081145 & 2.80 & 0.69 & & & & & & & & & & & \\
\hline 8081278 & 33.20 & 3.86 & 33.10 & 3.90 & & & 33.92 & 3.84 & & & 33.28 & 34.26 & 3.87 \\
\hline $\begin{array}{l}8081535 \\
8081853\end{array}$ & 42.60 & 4.58 & 42.62 & 4.58 & 42.29 & 4.66 & $\begin{array}{c}43.13 \\
6.76\end{array}$ & & 40.33 & & 42.63 & 44.02 & 4.55 \\
\hline 8085998 & 34.70 & 4.12 & 35.72 & 4.25 & 35.10 & 4.12 & 34.69 & & 34.21 & 4.56 & 35.66 & 37.83 & 4.04 \\
\hline 8086192 & 74.40 & 7.09 & 75.69 & 7.01 & 74.43 & 7.00 & 74.23 & 7.10 & & & 75.19 & 77.79 & 7.10 \\
\hline 8087067 & 100.40 & 8.93 & 99.96 & 8.98 & 100.08 & 9.07 & 100.43 & 9.01 & & & 100.03 & 101.49 & 8.98 \\
\hline 8088244 & 29.90 & 4.14 & 29.72 & 4.11 & & & 31.77 & 4.16 & & & & & \\
\hline 8094602 & 55.30 & 5.41 & 55.74 & 5.58 & 54.57 & 5.57 & 54.44 & 5.49 & & & 55.98 & 58.07 & 5.42 \\
\hline 8099073 & 42.50 & 4.49 & 42.89 & 4.50 & 42.34 & 4.48 & 43.32 & 4.43 & & & 43.06 & 46.01 & 4.45 \\
\hline 8120076 & 33.40 & 3.91 & 33.76 & 4.18 & & & 33.56 & & & & 34.18 & 35.06 & 3.94 \\
\hline 8123843 & 34.60 & 3.62 & 34.92 & 3.72 & 34.54 & 3.59 & 34.52 & 3.65 & & & 34.88 & 35.36 & 3.63 \\
\hline 8144529 & 38.90 & 4.41 & 38.70 & 4.36 & 38.73 & 4.43 & 39.02 & 4.40 & & & 39.28 & 40.54 & 4.36 \\
\hline 8154383 & 64.90 & 6.37 & & & 64.95 & 6.45 & 64.66 & 6.41 & 65.58 & 6.06 & 65.95 & 68.47 & 6.34 \\
\hline 8160590 & 34.00 & 4.04 & 33.77 & 4.04 & 33.29 & 4.09 & 34.09 & 3.95 & 30.98 & 3.35 & 33.86 & 35.89 & 4.15 \\
\hline
\end{tabular}


Table 7. continued.

\begin{tabular}{|c|c|c|c|c|c|c|c|c|c|c|c|c|c|}
\hline Star & $\begin{array}{l}\mathrm{COR} \\
v_{\max } \\
\mu \mathrm{Hz} \\
\end{array}$ & $\begin{array}{c}\mathrm{COR} \\
\Delta v \\
\mu \mathrm{Hz} \\
\end{array}$ & $\begin{array}{l}\text { CAN } \\
v_{\max } \\
\mu \mathrm{Hz} \\
\end{array}$ & $\begin{array}{c}\text { CAN } \\
\Delta v \\
\mu \mathrm{Hz}\end{array}$ & $\begin{array}{l}\mathrm{A} 2 \mathrm{Z} \\
v_{\max } \\
\mu \mathrm{Hz}\end{array}$ & $\begin{array}{c}\mathrm{A} 2 \mathrm{Z} \\
\Delta v \\
\mu \mathrm{Hz}\end{array}$ & $\begin{array}{l}\text { SYD } \\
v_{\max } \\
\mu \mathrm{Hz}\end{array}$ & $\begin{array}{c}\text { SYD } \\
\Delta v \\
\mu \mathrm{Hz}\end{array}$ & $\begin{array}{c}\text { DLB } \\
v_{\max } \\
\mu \mathrm{Hz}\end{array}$ & $\begin{array}{c}\text { DLB } \\
\Delta v \\
\mu \mathrm{Hz}\end{array}$ & $\begin{array}{c}\text { OCT I } \\
v_{\max } \\
\mu \mathrm{Hz}\end{array}$ & $\begin{array}{c}\text { OCT II } \\
v_{\max } \\
\mu \mathrm{Hz}\end{array}$ & $\begin{array}{c}\text { OCT } \\
\Delta v \\
\mu \mathrm{Hz}\end{array}$ \\
\hline 8164977 & 35.00 & 4.38 & 34.06 & 4.08 & 33.24 & 3.68 & 35.34 & & & & 34.99 & 36.74 & \\
\hline 8173148 & 34.40 & 4.19 & 33.97 & 4.18 & 34.01 & 4.29 & 34.49 & 4.17 & & & 34.48 & 35.46 & 4.25 \\
\hline 8173725 & 47.60 & 5.02 & & & 47.60 & 5.00 & 47.56 & & 48.87 & & 48.56 & 51.29 & 4.91 \\
\hline 8176543 & & & & & & & 13.12 & & & & & & \\
\hline 8192986 & & & & & & & & & 16.93 & 2.51 & & & \\
\hline 8195595 & 69.00 & 6.42 & 65.59 & 6.52 & 65.85 & 6.35 & 69.04 & 6.49 & & & 66.05 & 68.03 & 6.39 \\
\hline 8196436 & 37.50 & 4.17 & 40.12 & 4.16 & 38.50 & 4.17 & 37.84 & 4.20 & 36.17 & & 40.82 & 43.02 & 4.19 \\
\hline 8210100 & 40.90 & 4.46 & 40.93 & 4.44 & 41.59 & 4.35 & 41.25 & 4.47 & 35.43 & 4.44 & 41.62 & 43.57 & 4.38 \\
\hline 8210270 & 86.80 & 6.31 & & & & & & & & & & & \\
\hline 8212786 & 19.70 & 2.55 & 19.72 & 2.62 & 18.61 & 2.46 & 19.72 & 2.55 & & & 19.87 & 21.32 & 2.53 \\
\hline 8218419 & & & & & & & & & 1.76 & 0.46 & & & \\
\hline 8232851 & 45.80 & 4.74 & 46.19 & 4.69 & 45.72 & 4.70 & 45.58 & 4.80 & 44.51 & & 46.34 & 48.84 & 4.81 \\
\hline 8233917 & 30.00 & 3.76 & 30.04 & 3.55 & & & 29.99 & 3.87 & & & 30.09 & 31.13 & 3.79 \\
\hline 8235013 & 27.80 & 3.64 & 28.02 & 3.64 & & & 27.49 & & & & 28.34 & 30.49 & 3.66 \\
\hline 8241402 & & & & & 29.32 & 2.88 & 30.09 & & & & 30.07 & 31.17 & \\
\hline 8246635 & 40.40 & 4.49 & 39.64 & 4.71 & 40.47 & 4.46 & 41.08 & 4.47 & 37.29 & 4.29 & 40.75 & 43.09 & 4.35 \\
\hline 8248948 & 30.80 & 3.37 & 28.74 & 3.37 & 28.95 & 3.37 & 31.42 & 3.38 & & & 29.27 & 29.69 & 3.34 \\
\hline 8258311 & & & & & 35.15 & 3.64 & 37.39 & & & & 36.37 & 36.47 & \\
\hline 8260453 & 46.00 & 4.68 & 44.06 & 4.71 & & & & & & & 44.80 & 45.54 & \\
\hline 8278906 & 31.20 & 4.12 & 30.02 & 4.29 & & & 32.14 & & & & 31.17 & 31.77 & 4.12 \\
\hline 8284032 & 39.50 & 4.27 & 39.09 & 4.29 & 39.29 & 4.27 & 39.74 & 4.33 & & & 39.61 & 40.39 & 4.23 \\
\hline 8285025 & 14.70 & 2.02 & & & 15.50 & 2.07 & 15.05 & & & & 14.85 & 15.85 & \\
\hline 8293158 & 33.10 & 4.19 & 33.73 & 4.17 & 32.99 & 4.10 & 35.11 & 4.25 & & & 34.40 & 36.01 & 4.26 \\
\hline 8293686 & 10.80 & 1.52 & & & & & 10.98 & & & & & & \\
\hline 8300747 & 15.50 & 2.44 & 15.99 & 2.39 & 15.73 & 2.23 & 15.98 & & & & 16.32 & 17.38 & 2.45 \\
\hline 8307630 & 32.20 & 3.91 & 32.79 & 3.84 & 31.32 & 3.94 & 32.83 & 3.86 & 31.14 & 4.22 & 32.69 & 32.01 & 3.93 \\
\hline 8310792 & 125.90 & 8.09 & 114.39 & 8.27 & 110.86 & 8.28 & 107.12 & 8.30 & & & 111.86 & 120.81 & 8.55 \\
\hline 8343654 & 30.00 & 3.27 & 28.97 & 3.42 & 29.14 & 3.31 & 30.06 & 3.27 & & & 29.93 & 30.91 & 3.27 \\
\hline 8346238 & 31.50 & 3.76 & & & & & 30.78 & 3.62 & & & 31.36 & 34.08 & 3.79 \\
\hline 8350443 & 31.00 & 4.12 & & & & & 31.18 & & 26.36 & 3.12 & & & \\
\hline 8350645 & 54.30 & 5.21 & 53.43 & 5.29 & 53.92 & 5.15 & 54.54 & 5.30 & 47.18 & 5.29 & 54.24 & 56.43 & 5.18 \\
\hline 8359169 & 52.10 & 5.33 & 53.22 & 5.48 & 53.23 & 5.33 & 52.51 & 5.30 & & & 53.33 & 55.27 & 5.25 \\
\hline 8366239 & 182.40 & 13.75 & 181.11 & 13.70 & 180.63 & 13.78 & 186.43 & 13.73 & & & 184.17 & 186.59 & 13.66 \\
\hline 8377116 & 27.60 & 3.82 & 28.48 & 3.82 & & & 26.97 & 3.92 & & & 29.18 & 32.32 & 3.81 \\
\hline 8378462 & 89.00 & 7.31 & 89.06 & & 88.42 & 7.32 & 88.29 & 7.38 & 91.63 & & 89.65 & 91.60 & 7.27 \\
\hline 8378653 & 88.90 & 7.39 & 86.89 & 7.39 & 89.34 & 7.31 & 89.65 & & & & 89.81 & 92.91 & 7.40 \\
\hline 8381505 & & & & & & & 7.20 & & & & & & \\
\hline 8386921 & 27.10 & 3.56 & 27.04 & 3.58 & & & 27.46 & & & & 27.55 & 28.28 & 3.59 \\
\hline 8387412 & 6.60 & 1.06 & & & & & 6.56 & & & & & & \\
\hline 8396782 & 82.30 & 7.16 & 80.03 & 7.23 & 82.49 & 7.11 & 82.27 & 7.09 & & & 83.29 & 88.55 & 7.13 \\
\hline 8397081 & 72.20 & 5.88 & 67.43 & 5.88 & 68.95 & 5.71 & 70.79 & 5.83 & & & 70.67 & 73.56 & 5.88 \\
\hline 8410006 & 32.10 & 4.07 & 31.90 & 3.91 & & & 31.69 & 3.95 & & & 32.42 & 33.84 & \\
\hline 8410637 & & & & & & & 43.42 & 4.61 & & & & & \\
\hline 8411503 & & & & & & & 283.50 & & & & & & \\
\hline 8415501 & 32.00 & 3.68 & 31.80 & 3.59 & 31.51 & 3.62 & 32.27 & 3.60 & & & 31.97 & 32.57 & 3.63 \\
\hline 8431695 & 59.80 & 5.98 & 57.76 & 6.09 & 58.52 & 6.00 & 60.25 & 6.14 & & & 58.26 & 58.80 & 5.98 \\
\hline 8432239 & 51.50 & 4.95 & 51.68 & 4.95 & 51.09 & 5.00 & 51.41 & 4.93 & 47.57 & 5.17 & 51.77 & 54.96 & 4.94 \\
\hline 8454209 & 15.80 & 1.90 & 15.75 & 1.93 & & & 15.79 & 1.93 & 15.97 & & & & \\
\hline 8458519 & 31.00 & 3.86 & 31.71 & 3.86 & 31.04 & 3.82 & 30.71 & 3.97 & & & 32.02 & 33.75 & 3.85 \\
\hline 8461449 & 54.70 & 5.29 & 53.10 & 5.46 & 55.74 & 5.16 & 55.20 & 5.28 & & & 56.36 & 56.28 & 5.30 \\
\hline 8462934 & 72.90 & 6.25 & 70.73 & 6.70 & 72.24 & 6.31 & 73.32 & 6.30 & & & 72.72 & 74.55 & 6.24 \\
\hline 8475025 & 111.20 & 9.55 & 110.71 & 9.35 & 111.10 & 9.76 & 110.48 & 9.52 & & & 111.12 & 114.42 & 9.60 \\
\hline 8475511 & 13.50 & 2.08 & & & 13.31 & 1.97 & 13.46 & 1.98 & & & & & \\
\hline 8476245 & 10.70 & 1.80 & & & 12.82 & 1.48 & 11.71 & & 9.40 & & & & \\
\hline 8480097 & 192.00 & 15.62 & 197.28 & 15.32 & 200.78 & 15.48 & 195.69 & 15.41 & & & & & \\
\hline 8482522 & 34.00 & 3.44 & 33.30 & 3.44 & & & 33.20 & & & & 33.92 & 35.52 & \\
\hline 8495082 & 8.70 & 1.44 & & & & & 8.98 & & & & & & \\
\hline 8496527 & & & & & 33.99 & 3.93 & & & & & & & \\
\hline 8500704 & & & & & & & 26.28 & 4.04 & & & 26.81 & 28.22 & \\
\hline 8506788 & 31.00 & 4.32 & & & & & 31.13 & & & & 31.20 & 32.01 & 4.17 \\
\hline 8507397 & 35.30 & 4.35 & 33.83 & 4.38 & 34.42 & 4.36 & 36.27 & & 27.20 & & & & \\
\hline 8508931 & 55.00 & 4.98 & & & & & & & & & & & \\
\hline 8515390 & 56.00 & 5.25 & 54.64 & 5.42 & 54.75 & 5.28 & 56.34 & 5.41 & 53.36 & 5.55 & 55.02 & 56.67 & 5.23 \\
\hline 8518198 & 135.30 & 11.46 & 135.06 & 11.56 & 136.38 & 11.35 & 135.72 & 11.56 & & & 135.13 & 134.96 & 11.49 \\
\hline 8522040 & 47.50 & 4.74 & 48.03 & 4.80 & 47.05 & 4.77 & 48.15 & 4.74 & 47.44 & 5.18 & 48.15 & 49.58 & 4.72 \\
\hline
\end{tabular}


S. Hekker et al.: Comparison of global oscillation parameters from different methods

Table 7. continued.

\begin{tabular}{|c|c|c|c|c|c|c|c|c|c|c|c|c|c|}
\hline Star & $\begin{array}{l}\text { COR } \\
v_{\max } \\
\mu \mathrm{Hz}\end{array}$ & $\begin{array}{c}\text { COR } \\
\Delta v \\
\mu \mathrm{Hz}\end{array}$ & $\begin{array}{l}\text { CAN } \\
v_{\max } \\
\mu \mathrm{Hz}\end{array}$ & $\begin{array}{c}\text { CAN } \\
\Delta v \\
\mu \mathrm{Hz}\end{array}$ & $\begin{array}{l}\mathrm{A} 2 \mathrm{Z} \\
v_{\max } \\
\mu \mathrm{Hz}\end{array}$ & $\begin{array}{c}\mathrm{A} 2 \mathrm{Z} \\
\Delta v \\
\mu \mathrm{Hz}\end{array}$ & $\begin{array}{l}\text { SYD } \\
v_{\max } \\
\mu \mathrm{Hz}\end{array}$ & $\begin{array}{c}\text { SYD } \\
\Delta v \\
\mu \mathrm{Hz}\end{array}$ & $\begin{array}{l}\text { DLB } \\
v_{\max } \\
\mu \mathrm{Hz}\end{array}$ & $\begin{array}{c}\text { DLB } \\
\Delta v \\
\mu \mathrm{Hz}\end{array}$ & $\begin{array}{c}\text { OCT I } \\
v_{\max } \\
\mu \mathrm{Hz}\end{array}$ & $\begin{array}{c}\text { OCT II } \\
v_{\max } \\
\mu \mathrm{Hz}\end{array}$ & $\begin{array}{c}\text { OCT } \\
\Delta v \\
\mu \mathrm{Hz}\end{array}$ \\
\hline 8525359 & 57.50 & 5.78 & 57.66 & 5.57 & 57.17 & 5.74 & 57.78 & 5.58 & & & 57.42 & 59.33 & 5.77 \\
\hline 8526108 & 42.80 & 4.55 & 42.26 & 4.33 & 41.89 & 4.66 & 42.86 & 4.43 & 42.15 & & 42.70 & 44.40 & 4.64 \\
\hline 8539201 & 34.80 & 3.84 & 34.16 & 3.99 & 34.43 & 3.73 & 35.78 & & 33.70 & 3.53 & 34.70 & 35.82 & 3.81 \\
\hline 8540615 & 43.70 & 4.71 & 44.97 & 4.65 & 42.95 & 4.79 & 47.56 & 4.67 & & & 45.44 & 48.35 & 4.68 \\
\hline 8540767 & 28.40 & 3.12 & 28.43 & 3.12 & 27.27 & & 28.75 & & & & 28.86 & 28.90 & \\
\hline 8543727 & 4.40 & 0.81 & & & & & & & & & & & \\
\hline 8545504 & 106.20 & 9.42 & 107.95 & 9.30 & 107.60 & 9.32 & 105.70 & 9.28 & & & 108.13 & 107.73 & 9.27 \\
\hline 8546146 & 6.50 & 0.94 & & & & & 6.48 & & & & & & \\
\hline 8547390 & 44.70 & 4.71 & 43.10 & 4.85 & 42.84 & 4.57 & 44.91 & 4.69 & 39.76 & & 44.35 & 46.56 & 4.65 \\
\hline 8547469 & 29.20 & 3.97 & 29.26 & 3.89 & 28.90 & 3.65 & 29.52 & & & & 29.62 & 32.24 & 3.81 \\
\hline 8561238 & 51.80 & 5.41 & 54.20 & 5.41 & & & 51.14 & 5.37 & & & 55.75 & 58.30 & 5.34 \\
\hline 8583586 & 49.60 & 4.43 & 46.80 & 4.44 & 44.92 & 4.65 & 49.99 & 4.41 & 45.88 & & 47.99 & 46.05 & 4.45 \\
\hline 8611833 & 37.60 & 4.14 & 38.68 & 4.14 & 38.23 & 4.18 & 37.21 & 4.19 & & & 38.96 & 40.30 & 4.14 \\
\hline 8612520 & 41.90 & 4.24 & 41.05 & 4.28 & 40.63 & 4.17 & 41.96 & 4.25 & & & 41.80 & 44.24 & 4.22 \\
\hline 8612811 & 31.00 & 4.24 & 31.89 & 4.13 & 31.33 & 4.20 & 31.00 & & & & 32.04 & 33.64 & 4.26 \\
\hline 8617695 & & & & & & & 236.00 & & & & & & \\
\hline 8624155 & 80.20 & 7.02 & 80.08 & & 79.92 & 7.03 & 77.23 & & & & 82.09 & 84.00 & 7.08 \\
\hline 8628585 & 35.20 & 4.14 & 36.12 & 4.22 & 35.13 & 4.11 & 35.23 & 4.15 & & & 36.50 & 39.29 & 4.19 \\
\hline 8649099 & & & & & 41.02 & 4.41 & 42.59 & 4.44 & 41.38 & & & & \\
\hline 8669282 & 37.40 & 4.41 & 37.00 & 4.38 & 38.26 & 4.38 & 37.09 & 4.41 & 34.80 & 4.84 & 38.24 & 38.90 & 4.32 \\
\hline 8683589 & 15.40 & 2.32 & 15.62 & 2.35 & 15.34 & 2.18 & 15.51 & & & & 15.80 & 17.04 & \\
\hline 8686058 & 44.60 & 4.71 & 44.53 & 4.73 & 44.44 & 4.71 & 44.73 & & 46.09 & & 44.86 & 45.77 & 4.74 \\
\hline 8686389 & & & & & 23.22 & 2.37 & 23.91 & & & & 23.48 & 24.30 & 3.01 \\
\hline 8690009 & 28.00 & 3.72 & 28.82 & 3.60 & & & 28.14 & & & & 28.90 & 31.30 & \\
\hline 8702088 & & & & & & & & & 23.10 & 3.04 & & & \\
\hline 8707667 & 35.70 & 3.95 & 36.16 & 3.95 & 34.53 & 3.92 & 36.08 & 3.92 & & & 36.69 & 38.29 & 3.98 \\
\hline 8708654 & 51.90 & 5.33 & & & 54.99 & 5.26 & 51.71 & 5.36 & & & 55.19 & 58.11 & 5.33 \\
\hline 8718745 & 129.30 & 11.65 & 130.27 & 11.36 & 131.19 & 11.30 & 130.67 & 11.33 & 121.10 & & 129.29 & 127.78 & 11.50 \\
\hline 8736415 & 7.60 & 1.43 & & & & & 7.80 & & & & & & \\
\hline 8740371 & & & & & & & & & 7.09 & 1.39 & & & \\
\hline 8746834 & & & 56.10 & 5.83 & & & & & & & 55.24 & 54.31 & \\
\hline 8747415 & & & 137.08 & 11.32 & & & & & & & 137.26 & 142.89 & 11.30 \\
\hline 8748278 & 35.40 & 3.88 & 34.84 & 4.26 & 33.90 & 3.96 & 36.08 & & & & 35.05 & 35.18 & 3.89 \\
\hline 8753234 & 55.00 & 5.41 & 57.21 & 5.32 & 56.63 & 5.45 & 56.09 & 5.43 & 51.29 & 5.45 & 57.85 & 60.42 & 5.40 \\
\hline 8757215 & 35.20 & 4.22 & 36.17 & 4.29 & & & 35.51 & 4.28 & & & 36.33 & 37.64 & 4.22 \\
\hline 8758700 & 31.50 & 4.02 & 31.55 & 4.03 & 29.35 & & 32.37 & & & & 32.43 & 33.41 & 3.97 \\
\hline 8771414 & 38.90 & 4.24 & 40.00 & 4.39 & 39.02 & 4.17 & 38.65 & 4.22 & & & 40.41 & 42.04 & 4.25 \\
\hline 8803432 & 32.20 & 3.93 & 31.87 & 4.11 & & & 32.74 & 3.89 & & & 32.54 & 33.16 & 4.02 \\
\hline 8812397 & 70.70 & 6.67 & & & & & 69.55 & 6.70 & & & 70.14 & 72.56 & 6.80 \\
\hline 8813946 & 71.70 & 6.44 & & & & & & & & & & & \\
\hline 8818252 & & & & & & & 18.75 & 2.75 & & & 18.74 & 20.41 & 2.56 \\
\hline 8827646 & 33.70 & 4.02 & 32.48 & 4.42 & 32.19 & 3.99 & 34.07 & & & & & & \\
\hline 8827934 & 52.70 & 5.33 & 52.09 & 5.46 & 55.50 & 5.37 & 55.50 & 5.34 & & & 53.68 & 56.37 & 5.37 \\
\hline 8839028 & 27.80 & 3.26 & & & 26.89 & 3.67 & 28.36 & 3.38 & & & 27.72 & 28.70 & \\
\hline 8840004 & 11.60 & 1.60 & & & 10.91 & 1.50 & 11.46 & 1.58 & 11.13 & 1.87 & & & \\
\hline 8843610 & 41.80 & 4.35 & 40.78 & 4.39 & 41.77 & 4.40 & 41.92 & 4.30 & & & 41.47 & 43.00 & 4.42 \\
\hline 8845313 & 34.90 & 4.41 & & & & & 35.28 & & & & 37.14 & 39.26 & 4.38 \\
\hline 8845321 & & & & & & & 30.51 & & & & 31.81 & 34.24 & \\
\hline 8846187 & 47.40 & 4.95 & 48.28 & 4.95 & 48.13 & 5.00 & 47.86 & 4.93 & & & 48.43 & 49.78 & 5.02 \\
\hline 8867740 & 22.70 & 2.91 & 22.09 & 3.02 & 22.28 & & 22.96 & 2.95 & & & 22.71 & 23.21 & 2.89 \\
\hline 8873797 & 29.30 & 3.76 & 28.88 & 3.77 & 28.44 & 3.75 & 28.40 & 3.75 & & & 29.56 & 31.86 & 3.74 \\
\hline 8880420 & 18.70 & 2.69 & 19.59 & 2.68 & 18.30 & 2.41 & 18.78 & & & & 19.66 & 20.57 & \\
\hline 8907943 & 31.70 & 4.02 & 32.33 & 4.21 & 31.72 & 3.28 & 31.28 & & & & 32.86 & 34.83 & \\
\hline 8909673 & 43.90 & 4.68 & 44.28 & 4.56 & & & 43.69 & 4.66 & & & 44.69 & 46.26 & 4.60 \\
\hline 8911558 & 40.30 & 4.43 & 40.05 & 4.58 & 40.17 & 4.36 & 40.16 & 4.45 & 38.07 & 4.65 & 40.74 & 42.80 & 4.43 \\
\hline 8914504 & 33.80 & 4.17 & 33.32 & 4.16 & 34.26 & 3.70 & 34.44 & 4.16 & & & 34.33 & 35.88 & 4.19 \\
\hline 8934264 & & & & & & & 8.84 & & & & & & \\
\hline 8936084 & 30.00 & 4.52 & 30.67 & 4.31 & & & 30.26 & & & & 31.10 & 32.53 & 4.30 \\
\hline 8936339 & & & & & & & 44.19 & & & & 44.38 & 44.60 & 4.60 \\
\hline 8936409 & 6.80 & 0.91 & & & & & 7.00 & & & & & & \\
\hline 8941202 & 33.90 & 4.00 & 34.10 & 4.22 & 33.75 & 3.17 & 34.39 & 4.14 & & & 35.29 & 36.13 & 4.04 \\
\hline 8949653 & 18.50 & 2.60 & 18.94 & 2.55 & 18.78 & 2.10 & 19.69 & 2.55 & & & 19.26 & 19.61 & \\
\hline 8955683 & 45.80 & 4.71 & 47.45 & 4.61 & 46.43 & 4.68 & 45.75 & 4.82 & 43.98 & 4.96 & 47.90 & 50.35 & 4.72 \\
\hline 8962844 & 38.80 & 4.49 & 40.70 & 4.52 & 40.18 & 4.58 & 39.04 & 4.55 & & & 41.30 & 41.49 & 4.43 \\
\hline 9002344 & 43.50 & 4.38 & 42.44 & 4.40 & 42.15 & 4.27 & 44.54 & 4.43 & & & 42.87 & 44.92 & 4.32 \\
\hline
\end{tabular}


Table 7. continued.

\begin{tabular}{|c|c|c|c|c|c|c|c|c|c|c|c|c|c|}
\hline Star & $\begin{array}{c}\text { COR } \\
v_{\max } \\
\mu \mathrm{Hz}\end{array}$ & $\begin{array}{c}\text { COR } \\
\Delta v \\
\mu \mathrm{Hz}\end{array}$ & $\begin{array}{l}\text { CAN } \\
v_{\max } \\
\mu \mathrm{Hz}\end{array}$ & $\begin{array}{c}\text { CAN } \\
\Delta v \\
\mu \mathrm{Hz}\end{array}$ & $\begin{array}{l}\mathrm{A} 2 \mathrm{Z} \\
v_{\max } \\
\mu \mathrm{Hz}\end{array}$ & $\begin{array}{c}\mathrm{A} 2 \mathrm{Z} \\
\Delta v \\
\mu \mathrm{Hz}\end{array}$ & $\begin{array}{l}\text { SYD } \\
v_{\max } \\
\mu \mathrm{Hz}\end{array}$ & $\begin{array}{c}\text { SYD } \\
\Delta v \\
\mu \mathrm{Hz}\end{array}$ & $\begin{array}{l}\text { DLB } \\
v_{\max } \\
\mu \mathrm{Hz}\end{array}$ & $\begin{array}{c}\text { DLB } \\
\Delta v \\
\mu \mathrm{Hz}\end{array}$ & $\begin{array}{c}\text { OCT I } \\
v_{\max } \\
\mu \mathrm{Hz}\end{array}$ & $\begin{array}{c}\text { OCT II } \\
v_{\max } \\
\mu \mathrm{Hz}\end{array}$ & $\begin{array}{c}\text { OCT } \\
\Delta v \\
\mu \mathrm{Hz}\end{array}$ \\
\hline 9002884 & 4.80 & 0.92 & & & & & 4.85 & & & & & & \\
\hline 9009805 & 50.00 & 5.21 & 51.30 & 5.21 & 49.44 & 5.23 & 50.13 & 5.25 & & & 50.70 & 51.98 & 5.22 \\
\hline 9020419 & 32.60 & 3.86 & 32.97 & 4.03 & 32.33 & 2.71 & 32.93 & & & & 33.27 & 34.35 & \\
\hline 9021464 & 38.90 & 4.41 & 38.69 & 4.41 & 38.82 & 4.58 & 39.57 & 4.48 & & & 39.16 & 39.55 & 4.45 \\
\hline 9026209 & 9.20 & 1.34 & & & 9.25 & 1.35 & 9.38 & & & & & & \\
\hline 9027245 & 49.10 & 5.41 & 48.70 & 5.36 & 49.22 & 5.42 & 49.88 & 5.37 & & & 48.98 & 49.91 & 5.35 \\
\hline 9028293 & & & & & & & 62.36 & & & & 61.88 & 62.55 & 5.67 \\
\hline 9028697 & 59.00 & 5.88 & 58.46 & 5.94 & 58.78 & 5.79 & 59.58 & 5.95 & & & 58.81 & 60.03 & 5.86 \\
\hline 9072262 & 38.00 & 4.46 & 38.26 & 4.51 & 37.81 & & 38.13 & 4.53 & & & 38.46 & 40.31 & 4.52 \\
\hline 9075872 & 157.80 & 12.97 & 156.54 & 13.24 & 158.16 & 12.86 & 157.98 & 13.19 & & & 156.54 & 159.77 & 12.97 \\
\hline 9086505 & 30.50 & 3.95 & 30.40 & 4.10 & & & 30.53 & 4.09 & & & 30.93 & 32.54 & 3.97 \\
\hline 9088119 & 34.40 & 4.04 & 34.16 & 3.96 & & & 35.03 & 3.88 & 26.31 & 3.62 & 34.83 & 33.58 & 3.95 \\
\hline 9100325 & & & & & & & 5.29 & & & & & & \\
\hline 9110060 & 10.50 & 1.53 & & & & & 10.86 & 1.49 & & & & & \\
\hline 9112472 & 46.90 & 4.77 & 45.00 & 4.75 & 45.12 & 4.84 & 46.03 & 4.59 & & & 47.18 & 47.48 & 4.75 \\
\hline 9115334 & 68.40 & 6.03 & 63.71 & 6.42 & 65.89 & 6.00 & 66.36 & 6.09 & & & 68.82 & 70.36 & 6.05 \\
\hline 9138411 & 5.20 & 0.91 & & & & & & & & & & & \\
\hline 9139859 & 71.90 & 6.81 & 71.78 & 6.85 & 71.72 & 6.77 & 72.08 & 6.80 & & & 72.51 & 74.89 & 6.82 \\
\hline 9143834 & 15.70 & 2.32 & 15.74 & 2.27 & 15.82 & 2.36 & 15.69 & 2.29 & & & & & \\
\hline 9143924 & 10.20 & 1.51 & & & & & 10.25 & & & & & & \\
\hline 9145781 & & & & & & & 244.10 & & & & & & \\
\hline 9145952 & 32.70 & 3.88 & 32.32 & 3.80 & & & 33.65 & 3.69 & & & 32.54 & 33.74 & \\
\hline 9145955 & 124.90 & 10.91 & 131.86 & 10.90 & 131.24 & 10.97 & 131.04 & 10.95 & 128.72 & & 132.35 & 129.69 & 10.91 \\
\hline 9150223 & 36.30 & 4.38 & 35.44 & 4.39 & 35.30 & 4.24 & 36.12 & 4.37 & & & 36.55 & 37.93 & 4.29 \\
\hline 9151763 & 13.90 & 1.94 & & & & & 14.38 & 1.89 & & & & & \\
\hline 9157260 & & & & & 238.11 & 18.22 & 233.26 & 18.17 & & & & & \\
\hline 9159227 & 28.30 & 3.78 & 28.41 & 3.90 & 28.32 & 3.64 & 28.20 & 3.81 & & & 29.52 & 31.17 & 3.78 \\
\hline 9166023 & 19.10 & 2.38 & 18.69 & 2.39 & 19.47 & 2.35 & 19.25 & 2.40 & & & 18.85 & 19.04 & 2.35 \\
\hline 9173371 & 96.20 & 7.99 & 98.01 & 7.96 & 97.03 & 7.97 & 96.20 & 7.98 & 94.74 & & 98.72 & 101.42 & 8.03 \\
\hline 9176207 & 56.20 & 5.59 & 58.07 & 5.51 & 56.59 & 5.68 & 55.80 & 5.48 & & & 58.60 & 61.42 & 5.54 \\
\hline 9210413 & & & & & 230.74 & 19.82 & & & & & & & \\
\hline 9217456 & 10.90 & 1.52 & & & & & 10.56 & & & & & & \\
\hline 9230478 & 4.30 & 0.66 & & & & & & & & & & & \\
\hline 9240941 & 110.60 & 8.38 & & & 108.89 & 8.33 & 108.11 & 8.35 & 107.64 & & 110.39 & 113.68 & 8.38 \\
\hline 9246190 & 2.80 & 0.48 & & & & & & & & & & & \\
\hline 9265513 & 48.10 & 5.41 & 48.04 & 5.35 & 47.72 & 5.44 & 48.44 & 5.35 & & & 48.35 & 49.25 & 5.36 \\
\hline 9267654 & 118.60 & 10.42 & 118.03 & 10.30 & 118.30 & 10.17 & 117.98 & 10.26 & & & 117.98 & 121.69 & 10.34 \\
\hline 9268146 & 29.60 & 3.66 & 29.42 & 3.57 & 29.18 & 3.75 & 30.26 & 3.61 & & & 29.71 & 31.12 & 3.69 \\
\hline 9269772 & 55.30 & 5.93 & 57.17 & 6.06 & 56.14 & 5.94 & 54.71 & 5.91 & & & 57.68 & 60.32 & 5.90 \\
\hline 9300159 & 165.10 & 12.50 & 164.78 & 12.71 & 168.72 & 12.55 & 167.41 & 12.71 & 171.29 & & 166.18 & 165.20 & 12.61 \\
\hline 9307073 & 41.50 & 4.71 & 42.22 & 4.69 & 41.38 & 5.00 & 41.23 & 4.81 & 40.23 & & 42.33 & 44.45 & 4.75 \\
\hline 9327993 & & & & & & & & & & & 34.55 & 34.19 & 3.98 \\
\hline 9328135 & 54.60 & 5.78 & 55.52 & 5.79 & 55.05 & 5.73 & 54.49 & 5.78 & 41.54 & & 55.80 & 56.71 & 5.84 \\
\hline 9329200 & 76.10 & 6.81 & 76.36 & 6.87 & 75.79 & 6.94 & 76.48 & 6.81 & 75.46 & & 76.43 & 79.63 & 6.81 \\
\hline 9332840 & 38.40 & 4.58 & 39.64 & 4.49 & 39.32 & 4.31 & 39.37 & 4.47 & & & 39.66 & 43.03 & 4.44 \\
\hline 9333184 & 31.70 & 3.76 & 31.63 & 3.77 & 30.69 & 3.67 & 32.59 & 3.49 & & & 32.16 & 33.77 & \\
\hline 9333501 & 27.60 & 3.47 & 27.19 & 3.56 & 26.95 & 3.44 & 27.49 & 3.45 & & & 27.84 & 28.16 & \\
\hline 9340114 & 26.70 & 3.68 & & & & & 27.07 & & & & & & \\
\hline 9344639 & 7.00 & 1.14 & & & & & 6.92 & & & & & & \\
\hline 9346288 & 29.20 & 3.32 & 29.03 & 3.29 & 28.27 & 3.27 & 29.37 & 3.31 & & & 29.17 & 30.49 & 3.28 \\
\hline 9349632 & 96.70 & 7.72 & 93.34 & 7.73 & 98.35 & 7.67 & 98.18 & 7.80 & & & 95.76 & 95.42 & 7.76 \\
\hline 9349690 & 4.90 & 0.71 & & & & & 4.88 & & & & & & \\
\hline 9351617 & 37.40 & 4.38 & 39.05 & 4.43 & & & 37.07 & 4.35 & & & 38.74 & 42.09 & 4.43 \\
\hline 9364778 & & & & & & & 37.69 & & & & & & \\
\hline 9368427 & & & & & & & & & 21.01 & 2.63 & & & \\
\hline 9390293 & 30.30 & 3.80 & 31.03 & 3.80 & 29.90 & 3.69 & 30.83 & 3.73 & & & 31.30 & 33.08 & 3.79 \\
\hline 9392039 & 36.50 & 4.09 & 36.85 & 4.23 & 36.78 & 4.58 & 36.59 & 4.12 & & & 36.87 & 37.70 & 4.15 \\
\hline 9395535 & 21.70 & 2.96 & 22.03 & 2.93 & 21.57 & 2.47 & 21.68 & 2.93 & & & 22.36 & 23.24 & \\
\hline 9396363 & 42.90 & 4.55 & 42.95 & 4.62 & 43.90 & 4.58 & 43.00 & 4.61 & 39.27 & 4.79 & 42.97 & 43.85 & 4.46 \\
\hline 9414068 & 9.20 & 1.50 & & & & & 9.33 & & & & & & \\
\hline 9427247 & 124.30 & 8.70 & 112.59 & & & & 112.54 & 8.74 & & & 108.03 & 111.24 & 8.77 \\
\hline 9427889 & 37.40 & 3.70 & 38.47 & & 37.61 & 3.77 & 37.98 & 3.62 & & & 38.35 & 39.74 & 3.67 \\
\hline 9430402 & 70.00 & 6.08 & 68.13 & 6.08 & 69.98 & 6.07 & 68.86 & 6.19 & & & 70.22 & 72.03 & 6.05 \\
\hline 9430786 & 40.00 & 4.02 & 39.08 & 4.11 & 38.48 & 4.08 & 40.08 & 4.15 & 34.10 & & 39.45 & 38.20 & 4.01 \\
\hline 9447310 & 72.80 & 7.39 & 73.00 & 7.28 & 72.76 & 7.33 & 72.87 & 7.31 & 71.41 & 7.52 & 73.18 & 75.24 & 7.33 \\
\hline
\end{tabular}


S. Hekker et al.: Comparison of global oscillation parameters from different methods

Table 7. continued.

\begin{tabular}{|c|c|c|c|c|c|c|c|c|c|c|c|c|c|}
\hline Star & $\begin{array}{l}\mathrm{COR} \\
v_{\max } \\
\mu \mathrm{Hz}\end{array}$ & $\begin{array}{c}\text { COR } \\
\Delta v \\
\mu \mathrm{Hz}\end{array}$ & $\begin{array}{l}\text { CAN } \\
v_{\max } \\
\mu \mathrm{Hz}\end{array}$ & $\begin{array}{c}\text { CAN } \\
\Delta v \\
\mu \mathrm{Hz}\end{array}$ & $\begin{array}{l}\mathrm{A} 2 \mathrm{Z} \\
v_{\max } \\
\mu \mathrm{Hz}\end{array}$ & $\begin{array}{c}\mathrm{A} 2 \mathrm{Z} \\
\Delta v \\
\mu \mathrm{Hz}\end{array}$ & $\begin{array}{l}\text { SYD } \\
v_{\max } \\
\mu \mathrm{Hz}\end{array}$ & $\begin{array}{c}\text { SYD } \\
\Delta v \\
\mu \mathrm{Hz}\end{array}$ & $\begin{array}{l}\text { DLB } \\
v_{\max } \\
\mu \mathrm{Hz}\end{array}$ & $\begin{array}{c}\text { DLB } \\
\Delta v \\
\mu \mathrm{Hz}\end{array}$ & $\begin{array}{c}\text { OCT I } \\
v_{\max } \\
\mu \mathrm{Hz}\end{array}$ & $\begin{array}{c}\text { OCT II } \\
v_{\max } \\
\mu \mathrm{Hz}\end{array}$ & $\begin{array}{c}\text { OCT } \\
\Delta v \\
\mu \mathrm{Hz}\end{array}$ \\
\hline 9451434 & 27.60 & 3.66 & & & 27.54 & 3.57 & 27.56 & 3.54 & 26.94 & 3.55 & 28.38 & 30.33 & \\
\hline 9456598 & 40.50 & 4.32 & 40.66 & 4.53 & 41.36 & 4.32 & 40.83 & 4.51 & & & 41.72 & 42.97 & 4.35 \\
\hline 9470055 & & & & & & & 8.73 & & 8.72 & & & & \\
\hline 9474021 & 2.20 & 0.42 & & & & & & & 2.61 & 0.59 & & & \\
\hline 9475697 & 116.80 & 9.82 & 113.50 & 9.83 & 117.20 & 9.63 & 116.94 & 9.87 & 117.67 & & 112.69 & 113.83 & 9.81 \\
\hline 9508346 & 12.40 & 1.84 & & & & & 12.59 & 1.81 & & & & & \\
\hline 9508595 & 29.20 & 4.27 & 28.02 & 4.28 & 27.92 & 3.96 & 30.31 & 4.13 & & & 29.19 & 29.76 & 4.01 \\
\hline 9511938 & 48.70 & 4.91 & & & 48.01 & 4.91 & 48.89 & 4.90 & 44.14 & & 48.94 & 49.35 & 4.88 \\
\hline 9517003 & 8.30 & 1.32 & & & & & 8.32 & & & & & & \\
\hline 9517542 & 20.50 & 2.69 & 20.58 & 2.71 & 21.21 & 2.66 & 20.66 & 2.78 & 17.64 & & 20.79 & 21.17 & 2.71 \\
\hline 9520156 & 31.30 & 4.38 & 31.61 & 3.85 & & & 32.89 & & & & 31.85 & 32.33 & \\
\hline 9540226 & 26.20 & 3.17 & & & & & & & & & & & \\
\hline 9542075 & & & & & 26.66 & 3.84 & 27.15 & 4.06 & & & 28.18 & 30.03 & 3.94 \\
\hline 9542218 & 56.00 & 5.78 & 57.35 & 5.77 & 56.38 & 5.73 & 56.02 & 5.80 & & & 57.98 & 58.22 & 5.78 \\
\hline 9551036 & 54.00 & 5.73 & 54.25 & 5.70 & 53.58 & 5.81 & 53.89 & 5.72 & & & 54.05 & 54.71 & 5.73 \\
\hline 9569462 & 26.50 & 3.62 & 26.79 & 3.62 & 26.84 & & 26.82 & 3.52 & & & 27.95 & 29.74 & \\
\hline 9570409 & 29.80 & 3.66 & 30.28 & 3.55 & 28.64 & 3.68 & 29.97 & & & & & & \\
\hline 9574650 & 105.70 & 9.55 & 105.88 & 9.55 & 105.63 & 9.55 & 105.61 & 9.61 & & & 105.68 & 106.84 & 9.54 \\
\hline 9575803 & & & & & 260.76 & 20.54 & 283.28 & & & & & & \\
\hline 9579860 & 57.40 & 5.93 & 55.65 & 5.94 & 55.79 & 5.77 & 57.55 & 5.94 & & & 56.33 & 56.13 & 5.85 \\
\hline 9583430 & 101.40 & 7.72 & 102.73 & 7.78 & 101.29 & 7.94 & 101.03 & 7.89 & & & 103.11 & 103.63 & 7.77 \\
\hline 9589500 & 106.30 & 8.28 & 101.74 & & 102.58 & 8.18 & 104.14 & 8.42 & & & 103.51 & 103.69 & 8.25 \\
\hline 9589638 & 41.30 & 4.38 & 41.37 & 4.40 & 41.13 & 4.33 & 41.50 & 4.38 & & & 41.44 & 43.08 & 4.38 \\
\hline 9596106 & 21.80 & 2.69 & 21.77 & 2.66 & 21.55 & 2.69 & 22.37 & 2.65 & 22.84 & 3.11 & 21.62 & 22.23 & 2.64 \\
\hline 9611634 & 27.90 & 3.47 & 28.42 & 3.46 & 27.80 & 3.44 & 28.06 & 3.44 & & & 28.25 & 30.26 & \\
\hline 9631782 & 64.90 & 6.49 & 64.43 & 6.65 & 64.51 & 6.67 & 64.97 & 6.58 & & & 64.89 & 67.62 & 6.47 \\
\hline 9635388 & 36.50 & 4.32 & & & 37.81 & 4.30 & 37.63 & 4.39 & 32.40 & 4.20 & & & \\
\hline 9635603 & 31.80 & 3.97 & & & & & 32.64 & & & & 32.05 & 32.52 & 3.95 \\
\hline 9635649 & 198.50 & 14.94 & 206.20 & 15.17 & 200.54 & 14.87 & 204.45 & 15.22 & & & 206.32 & 201.52 & 15.01 \\
\hline 9640886 & 33.20 & 4.00 & 33.18 & 4.02 & 33.44 & 3.85 & 33.80 & 4.01 & 27.33 & 3.62 & 33.76 & 35.89 & 3.82 \\
\hline 9641772 & 34.50 & 4.12 & 35.18 & 4.25 & 34.23 & 4.09 & 34.29 & 4.14 & 33.62 & 4.28 & 35.51 & 37.18 & 4.04 \\
\hline 9650046 & 66.50 & 6.03 & 67.80 & 6.03 & 65.98 & 6.00 & 67.42 & 6.02 & & & 67.73 & 68.71 & 6.03 \\
\hline 9650527 & 54.20 & 5.29 & 52.43 & 5.27 & 52.85 & 5.24 & 54.29 & 5.33 & & & 52.81 & 53.93 & 5.29 \\
\hline 9652494 & 42.90 & 4.55 & 41.43 & 4.58 & 42.55 & 4.52 & 43.62 & 4.62 & & & 42.43 & 43.11 & 4.53 \\
\hline 9655167 & & & 101.98 & 8.23 & & & & & & & & & \\
\hline 9657639 & & & & & & & 27.88 & & & & & & \\
\hline 9658002 & 37.90 & 4.12 & 35.28 & 4.05 & 35.08 & 4.15 & 38.18 & 4.19 & 30.92 & 4.02 & 37.23 & 38.02 & 4.11 \\
\hline 9666718 & 7.10 & 1.25 & & & & & & & & & & & \\
\hline 9674113 & 30.30 & 4.07 & 30.59 & 3.62 & & & 30.80 & 3.86 & 29.77 & 3.52 & 30.81 & 32.04 & 3.90 \\
\hline 9704774 & 33.70 & 4.27 & 33.96 & 4.17 & 34.44 & 4.06 & 33.38 & 4.18 & & & 34.31 & 35.91 & 4.21 \\
\hline 9705636 & 33.30 & 4.00 & 32.38 & 4.04 & 35.12 & 3.58 & 33.52 & & & & 33.40 & 34.78 & 3.92 \\
\hline 9705687 & 71.60 & 6.61 & 72.62 & & 74.44 & 6.57 & 72.00 & 6.68 & 70.17 & & 73.20 & 75.02 & 6.62 \\
\hline 9706194 & 25.50 & 3.35 & 26.20 & 3.33 & 25.56 & 3.36 & 25.84 & 3.31 & & & 26.34 & 27.35 & 3.29 \\
\hline 9711231 & 47.20 & 5.02 & 47.97 & 5.02 & 48.02 & 5.00 & 47.80 & 5.12 & 42.66 & 5.08 & 48.01 & 47.63 & 4.99 \\
\hline 9711349 & 15.30 & 2.11 & 15.03 & 2.14 & 15.42 & 2.16 & 15.42 & 2.08 & & & & & \\
\hline 9711492 & 49.40 & 5.02 & 51.07 & 5.29 & 50.77 & 5.00 & 49.73 & 5.07 & 54.38 & 5.31 & 51.98 & 54.26 & 5.01 \\
\hline 9711510 & 52.30 & 5.02 & 50.74 & 5.03 & 51.37 & 5.11 & 52.41 & 5.08 & & & 51.94 & 54.66 & 5.04 \\
\hline 9716866 & 32.20 & 3.72 & 33.33 & 3.73 & 32.50 & & 32.39 & 3.79 & & & 33.58 & 35.04 & 3.74 \\
\hline 9724324 & 39.70 & 4.17 & 39.76 & 4.23 & 40.23 & 4.09 & 40.13 & & 38.70 & 4.23 & 40.28 & 41.86 & 4.10 \\
\hline 9726045 & 3.30 & 0.67 & & & & & & & & & & & \\
\hline 9728845 & 27.10 & 3.21 & 27.59 & 3.12 & & & 27.17 & 3.14 & 26.41 & 3.39 & 27.97 & 30.55 & 3.14 \\
\hline 9761597 & 58.60 & 5.83 & 59.71 & 5.84 & 58.65 & 5.78 & 58.64 & 5.83 & & & 59.39 & 61.96 & 5.71 \\
\hline 9761625 & 9.10 & 1.25 & & & & & 9.31 & & & & & & \\
\hline 9767051 & 20.70 & 2.83 & 20.99 & 2.80 & 20.00 & & 20.52 & 2.83 & & & 21.01 & 23.61 & 2.85 \\
\hline 9767815 & 81.70 & 8.09 & 83.11 & 8.07 & 82.58 & 7.97 & 82.15 & 8.15 & & & 83.11 & 85.13 & 8.04 \\
\hline 9771905 & & & & & & & 12.15 & 1.78 & & & & & \\
\hline 9777536 & 45.70 & 4.74 & 45.21 & 4.72 & & & 46.28 & & & & 46.01 & 46.76 & 4.67 \\
\hline 9778288 & 51.60 & 5.09 & 54.25 & 5.21 & 50.82 & 5.23 & 52.60 & 5.11 & & & 55.66 & 61.46 & 5.13 \\
\hline 9780183 & 30.30 & 3.78 & & & & & 31.32 & & 27.19 & 3.54 & 30.41 & 31.32 & 3.77 \\
\hline 9783807 & & & & & & & & & 0.91 & 0.39 & & & \\
\hline 9791929 & 43.90 & 4.58 & & & & & & & & & & & \\
\hline 9814943 & 31.20 & 3.14 & 31.27 & 3.09 & 31.28 & 3.29 & 31.87 & & & & 31.25 & 32.98 & 3.12 \\
\hline 9818513 & 32.70 & 3.88 & 32.87 & 3.90 & 32.94 & 3.84 & 33.30 & 3.90 & & & 33.12 & 35.52 & 3.87 \\
\hline 9818604 & 30.70 & 3.56 & 31.12 & 3.51 & 30.81 & 3.49 & 30.93 & 3.52 & 29.15 & 3.83 & 31.09 & 32.15 & 3.55 \\
\hline 9825822 & 5.60 & 0.97 & & & & & 5.59 & & 5.16 & 1.01 & & & \\
\hline
\end{tabular}


Table 7. continued.

\begin{tabular}{|c|c|c|c|c|c|c|c|c|c|c|c|c|c|}
\hline Star & $\begin{array}{c}\text { COR } \\
v_{\max } \\
\mu \mathrm{Hz}\end{array}$ & $\begin{array}{c}\text { COR } \\
\Delta v \\
\mu \mathrm{Hz}\end{array}$ & $\begin{array}{l}\text { CAN } \\
v_{\max } \\
\mu \mathrm{Hz}\end{array}$ & $\begin{array}{c}\text { CAN } \\
\Delta v \\
\mu \mathrm{Hz}\end{array}$ & $\begin{array}{l}\mathrm{A} 2 \mathrm{Z} \\
v_{\max } \\
\mu \mathrm{Hz}\end{array}$ & $\begin{array}{c}\mathrm{A} 2 \mathrm{Z} \\
\Delta v \\
\mu \mathrm{Hz}\end{array}$ & $\begin{array}{l}\text { SYD } \\
v_{\max } \\
\mu \mathrm{Hz}\end{array}$ & $\begin{array}{c}\text { SYD } \\
\Delta v \\
\mu \mathrm{Hz}\end{array}$ & $\begin{array}{c}\text { DLB } \\
v_{\max } \\
\mu \mathrm{Hz}\end{array}$ & $\begin{array}{c}\overline{\mathrm{DLB}} \\
\Delta v \\
\mu \mathrm{Hz}\end{array}$ & $\begin{array}{c}\text { OCT I } \\
v_{\max } \\
\mu \mathrm{Hz}\end{array}$ & $\begin{array}{c}\text { OCT II } \\
v_{\max } \\
\mu \mathrm{Hz}\end{array}$ & $\begin{array}{c}\mathrm{OCT} \\
\Delta v \\
\mu \mathrm{Hz}\end{array}$ \\
\hline 9835436 & 24.50 & 3.12 & 24.82 & 2.90 & 24.12 & 3.01 & 24.30 & & & & & & \\
\hline 9835626 & 56.00 & 5.46 & 55.60 & 5.42 & 55.13 & 5.45 & 56.19 & 5.41 & 53.82 & & 55.71 & 56.10 & 5.46 \\
\hline 9838978 & 33.00 & 3.86 & 33.13 & 4.09 & & & 33.28 & & & & 33.25 & 33.11 & \\
\hline 9852625 & 14.40 & 2.22 & & & & & 14.50 & & & & & & \\
\hline 9882316 & & & & & 186.38 & 13.45 & 184.07 & 13.67 & & & 186.26 & 175.48 & 13.63 \\
\hline 9882550 & 36.00 & 4.27 & 35.60 & 4.18 & 35.49 & & 36.65 & & & & 36.58 & 38.30 & 4.20 \\
\hline 9884818 & 34.40 & 4.32 & 35.04 & 4.26 & 35.05 & 4.24 & 34.81 & & & & 35.24 & 37.05 & 4.23 \\
\hline 9896174 & & & & & 250.00 & 17.66 & 254.12 & & & & & & \\
\hline 9896877 & 9.30 & 1.61 & & & & & 9.60 & & & & & & \\
\hline 9902962 & 74.70 & 6.74 & 73.06 & 7.05 & 74.71 & 6.76 & 74.89 & 7.14 & & & 74.01 & 74.28 & 6.72 \\
\hline 9903290 & 4.90 & 0.93 & & & & & & & 4.48 & 0.91 & & & \\
\hline 9933754 & 33.10 & 4.04 & 33.16 & 4.05 & & & 33.05 & 4.11 & & & 33.90 & 35.19 & 4.04 \\
\hline 9941242 & 15.60 & 2.41 & 15.82 & 2.47 & & & 15.76 & 2.34 & & & & & \\
\hline 9942071 & 34.60 & 3.91 & 34.43 & 3.92 & 34.02 & 4.12 & 36.30 & & & & 35.36 & 36.54 & 3.94 \\
\hline 9942816 & 39.90 & 4.41 & 40.38 & 4.67 & & & 41.70 & & & & 40.78 & 42.80 & 4.39 \\
\hline 9945290 & 37.00 & 4.30 & 36.76 & 4.30 & 36.71 & 4.35 & 36.60 & 4.20 & & & 37.75 & 39.05 & 4.26 \\
\hline 9965582 & 7.70 & 1.13 & & & & & 7.60 & & & & & & \\
\hline 9967351 & 61.20 & 5.73 & 60.64 & 5.69 & 60.35 & 5.85 & 61.41 & 5.70 & 60.66 & & 60.54 & 61.30 & 5.70 \\
\hline 9968040 & 43.50 & 4.91 & 43.32 & 4.97 & 43.13 & 4.93 & 43.60 & 5.00 & & & 43.33 & 43.79 & 4.89 \\
\hline 9992544 & 28.10 & 3.42 & 28.44 & 3.46 & 28.16 & 3.41 & 28.21 & 3.45 & 28.93 & 3.89 & 28.45 & 28.54 & 3.44 \\
\hline 9994798 & 39.90 & 4.46 & 40.20 & 4.41 & 40.19 & 4.46 & 40.66 & 4.45 & 31.66 & 3.98 & 40.78 & 42.36 & 4.48 \\
\hline 9995464 & & & 29.20 & 3.85 & & & & & & & 29.94 & 30.95 & 3.98 \\
\hline 10004975 & 3.50 & 0.64 & & & & & 3.32 & & & & & & \\
\hline 10006158 & & & & & & & & & & & 30.92 & 32.42 & 4.07 \\
\hline 10010946 & 42.80 & 4.52 & 42.84 & 4.72 & 42.94 & 4.54 & 42.95 & 4.72 & 36.77 & 4.50 & 42.69 & 43.37 & 4.56 \\
\hline 10011329 & 35.70 & 3.80 & 36.13 & 3.81 & 35.58 & 3.88 & 35.75 & 3.79 & 32.51 & 3.34 & 36.31 & 37.72 & 3.79 \\
\hline 10016093 & 31.40 & 3.86 & 31.47 & 3.81 & 31.01 & 3.89 & 31.84 & 3.79 & & & 31.98 & 33.20 & \\
\hline 10031856 & 20.90 & 2.55 & 21.15 & 2.55 & 20.93 & 2.53 & 21.05 & 2.52 & & & 21.11 & 22.77 & \\
\hline 10034623 & 28.90 & 4.02 & 28.93 & 4.15 & & & 28.77 & & & & 29.46 & 31.62 & 3.92 \\
\hline 10053224 & 27.00 & 3.66 & & & 25.80 & 2.56 & 27.20 & & & & & & \\
\hline 10055061 & & & & & & & & & & & 16.08 & 16.35 & \\
\hline $\begin{array}{l}10055196 \\
10055660\end{array}$ & 109.30 & 8.81 & 107.45 & 8.75 & 108.04 & 9.00 & $\begin{array}{c}107.58 \\
021\end{array}$ & 8.77 & & & 108.11 & 111.08 & 8.83 \\
\hline 10055700 & 34.90 & 4.17 & 35.18 & 4.17 & 36.91 & 4.13 & 34.81 & & & & & & \\
\hline 10062594 & 42.80 & 4.71 & 43.20 & 4.79 & 43.07 & 4.81 & 43.01 & 4.79 & & & 43.18 & 44.08 & 4.72 \\
\hline 10068801 & 11.50 & 1.60 & & & & & 11.55 & 1.61 & 9.67 & & & & \\
\hline 10068810 & 49.80 & 5.37 & 50.04 & 5.37 & 49.68 & 5.38 & 49.96 & 5.40 & 44.46 & & 50.01 & 51.03 & 5.38 \\
\hline 10068892 & & & 14.76 & 2.08 & & & & & & & 14.91 & 15.66 & \\
\hline 10070717 & 31.70 & 4.07 & 32.47 & 4.15 & 35.04 & 4.05 & 31.50 & 4.14 & & & 32.77 & 34.10 & 4.03 \\
\hline 10080974 & 42.50 & 4.58 & 42.27 & 4.61 & 42.10 & 4.58 & 42.90 & 4.32 & & & 42.89 & 44.88 & 4.51 \\
\hline 10089758 & 6.00 & 1.47 & & & & & & & & & & & \\
\hline 10090400 & 32.50 & 4.00 & 33.08 & 4.02 & 32.31 & 3.68 & 32.78 & & & & 33.13 & 35.28 & 3.89 \\
\hline 10095409 & 40.10 & 4.14 & 39.51 & 4.10 & 38.82 & 4.21 & 40.77 & 4.22 & & & 39.86 & 38.99 & 4.19 \\
\hline 10118422 & 45.50 & 4.68 & 44.63 & 4.62 & 44.01 & 4.74 & 45.60 & 4.81 & & & 44.91 & 45.35 & 4.80 \\
\hline 10118929 & 41.60 & 4.27 & 40.96 & 4.30 & 41.65 & 4.31 & 42.06 & 4.29 & 36.85 & & 41.83 & 43.64 & 4.27 \\
\hline 10122158 & 203.10 & 14.03 & 187.42 & 14.46 & 203.82 & 14.30 & 185.85 & 14.42 & & & 192.30 & 193.17 & 14.36 \\
\hline 10123207 & 161.20 & 14.03 & 161.20 & 13.69 & 161.82 & 13.78 & 161.06 & 13.74 & & & 161.48 & 163.12 & 13.72 \\
\hline 10123573 & 34.70 & 3.91 & 34.22 & 4.29 & & & 35.93 & & & & 35.32 & 36.11 & \\
\hline 10124436 & 48.40 & 4.95 & 48.34 & 4.89 & 47.53 & 4.87 & 48.62 & 4.92 & 47.23 & & 48.32 & 47.13 & 4.92 \\
\hline 10136291 & 32.60 & 4.09 & 32.84 & 4.06 & 32.58 & 3.96 & 32.79 & 4.08 & & & 33.35 & 35.14 & 4.07 \\
\hline 10145279 & 31.00 & 3.64 & 28.81 & 3.62 & 29.70 & & 31.33 & 3.61 & & & 30.33 & 30.22 & 3.45 \\
\hline 10161943 & 62.10 & 6.31 & 61.83 & 6.29 & 61.78 & 6.32 & 62.01 & 6.27 & & & 61.78 & 62.24 & 6.33 \\
\hline 10162765 & 84.30 & 6.67 & & & 82.46 & 6.91 & 81.77 & 6.86 & & & & & \\
\hline 10186053 & 32.20 & 3.84 & & & 32.16 & 3.80 & 32.37 & & 31.75 & 4.14 & 32.72 & 33.88 & \\
\hline 10186608 & 32.70 & 4.19 & & & 32.32 & 3.95 & 32.52 & & 31.17 & 3.71 & 33.15 & 34.31 & 4.13 \\
\hline 10188141 & & & 34.66 & 4.32 & & & & & & & 35.83 & 37.90 & 4.21 \\
\hline 10188964 & 37.30 & 4.04 & 38.35 & 3.93 & & & 37.69 & & & & 38.33 & 39.56 & 4.16 \\
\hline 10189920 & 25.30 & 3.54 & 25.10 & 3.76 & & & 25.59 & & & & 25.89 & 28.41 & \\
\hline 10190733 & 29.50 & 3.78 & 29.74 & 3.87 & 29.85 & 3.74 & 29.25 & 3.77 & & & 30.50 & 32.53 & 3.77 \\
\hline 10192458 & 118.10 & 10.42 & 117.45 & 10.40 & 117.95 & 10.45 & 118.23 & 10.48 & & & 117.29 & 119.04 & 10.35 \\
\hline 10196240 & 34.50 & 4.09 & 34.45 & 4.18 & 34.33 & 4.15 & 34.57 & 4.14 & 33.43 & 4.16 & 35.06 & 37.36 & 4.10 \\
\hline 10199187 & 17.30 & 2.46 & 17.54 & 2.42 & & & 17.29 & & & & 17.69 & 18.85 & 2.47 \\
\hline 10200377 & 139.90 & 12.28 & 143.14 & 12.38 & 143.74 & 11.90 & 143.86 & 12.49 & & & 143.09 & 141.87 & 12.30 \\
\hline 10202184 & 157.30 & 12.73 & 157.12 & 12.73 & 158.13 & 12.70 & 156.78 & 12.68 & & & 157.42 & 160.67 & 12.66 \\
\hline 10207321 & 64.70 & 6.67 & 65.63 & 6.67 & 65.03 & 6.71 & 64.77 & 6.66 & & & 65.14 & 64.27 & 6.63 \\
\hline
\end{tabular}


Table 7. continued.

\begin{tabular}{|c|c|c|c|c|c|c|c|c|c|c|c|c|c|}
\hline Star & $\begin{array}{l}\text { COR } \\
v_{\max } \\
\mu \mathrm{Hz}\end{array}$ & $\begin{array}{c}\mathrm{COR} \\
\Delta v \\
\mu \mathrm{Hz}\end{array}$ & $\begin{array}{l}\text { CAN } \\
v_{\max } \\
\mu \mathrm{Hz}\end{array}$ & $\begin{array}{c}\text { CAN } \\
\Delta v \\
\mu \mathrm{Hz}\end{array}$ & $\begin{array}{l}\mathrm{A} 2 \mathrm{Z} \\
v_{\max } \\
\mu \mathrm{Hz}\end{array}$ & $\begin{array}{c}\mathrm{A} 2 \mathrm{Z} \\
\Delta v \\
\mu \mathrm{Hz}\end{array}$ & $\begin{array}{l}\text { SYD } \\
v_{\max } \\
\mu \mathrm{Hz}\end{array}$ & $\begin{array}{c}\text { SYD } \\
\Delta v \\
\mu \mathrm{Hz}\end{array}$ & $\begin{array}{l}\text { DLB } \\
v_{\max } \\
\mu \mathrm{Hz}\end{array}$ & $\begin{array}{c}\text { DLB } \\
\Delta v \\
\mu \mathrm{Hz}\end{array}$ & $\begin{array}{c}\text { OCT I } \\
v_{\max } \\
\mu \mathrm{Hz}\end{array}$ & $\begin{array}{c}\text { OCT II } \\
v_{\max } \\
\mu \mathrm{Hz}\end{array}$ & $\begin{array}{c}\text { OCT } \\
\Delta v \\
\mu \mathrm{Hz}\end{array}$ \\
\hline 10213931 & 43.50 & 4.55 & 42.57 & 4.53 & 42.96 & 4.56 & 44.00 & 4.54 & 39.73 & 4.35 & 43.12 & 43.59 & 4.53 \\
\hline 10220213 & & & 228.21 & 16.41 & & & 234.24 & & & & & & \\
\hline 10221370 & 43.30 & 4.52 & 42.13 & 4.65 & 42.67 & 4.56 & 43.54 & 4.52 & & & 43.28 & 44.34 & 4.52 \\
\hline 10228836 & 54.20 & 5.21 & 53.76 & 5.37 & & & 54.48 & 5.26 & & & 55.09 & 57.46 & 5.22 \\
\hline 10231806 & 9.70 & 1.48 & & & 9.40 & 1.51 & 10.15 & 1.47 & 9.81 & 1.28 & & & \\
\hline 10252281 & 30.70 & 4.00 & 31.36 & 4.00 & & & 30.24 & 4.10 & & & 31.84 & 33.27 & 4.00 \\
\hline 10252692 & 30.10 & 4.07 & 30.31 & 4.30 & & & 30.14 & & 26.86 & 3.49 & 30.08 & 30.75 & \\
\hline 10254023 & 22.50 & 3.14 & 22.79 & 3.14 & 22.36 & 3.20 & 22.77 & 3.11 & & & 23.03 & 23.79 & 3.23 \\
\hline 10257278 & 149.30 & 12.28 & 150.12 & 12.18 & 150.04 & 12.42 & 149.48 & 12.18 & & & 150.34 & 153.86 & 12.21 \\
\hline 10257419 & 91.00 & 8.28 & 91.31 & 8.39 & & & 91.15 & 8.41 & & & 91.75 & 93.38 & 8.30 \\
\hline 10257683 & 31.00 & 3.86 & 31.36 & 3.74 & 30.76 & 3.60 & 31.00 & 3.79 & 30.50 & 3.82 & 31.46 & 32.39 & 4.17 \\
\hline 10267242 & 27.70 & 2.72 & 27.27 & 2.72 & & & 27.81 & & & & 28.31 & 29.24 & \\
\hline 10271721 & 9.50 & 1.34 & & & 9.77 & 1.32 & 9.68 & 1.39 & & & & & \\
\hline 10286378 & 30.40 & 3.95 & 31.22 & 4.15 & 31.29 & 3.53 & 30.38 & 4.22 & & & 31.33 & 32.93 & 4.03 \\
\hline 10286616 & 33.60 & 4.24 & 33.80 & 4.25 & & & 34.07 & & & & 34.02 & 36.00 & 4.20 \\
\hline 10290315 & 39.80 & 4.27 & 39.06 & 4.19 & & & 40.35 & & & & 39.46 & 40.39 & 4.31 \\
\hline 10290718 & & & & & & & 8.27 & & & & & & \\
\hline 10293335 & 5.00 & 1.13 & & & & & 5.04 & & & & & & \\
\hline 10295060 & 8.10 & 1.17 & & & & & 7.96 & & & & & & \\
\hline 10297313 & 98.40 & 7.99 & 97.89 & 8.27 & 100.40 & 8.18 & 98.68 & 8.15 & & & 102.04 & 102.37 & 8.03 \\
\hline 10319045 & 5.30 & 0.92 & & & & & 5.61 & & & & & & \\
\hline 10319709 & 14.20 & 2.12 & & & 14.78 & 2.10 & 14.38 & & & & & & \\
\hline 10320170 & 24.30 & 2.96 & 24.44 & 3.06 & 24.48 & & 24.54 & 2.95 & & & 25.11 & 25.65 & 2.98 \\
\hline 10323222 & 46.60 & 4.84 & 47.39 & 4.84 & 46.60 & 4.80 & 46.44 & 4.86 & & & 47.36 & 48.50 & 4.82 \\
\hline 10328946 & 33.60 & 4.14 & 33.39 & 4.12 & & & 33.92 & 4.09 & & & 33.84 & 33.63 & \\
\hline 10332186 & 22.60 & 3.03 & 22.92 & 3.18 & 22.54 & 3.03 & 23.16 & 3.05 & & & 23.15 & 23.82 & 3.00 \\
\hline 10337695 & & & & & & & & & 22.57 & 3.33 & & & \\
\hline 10339590 & 3.30 & 0.61 & & & & & & & & & & & \\
\hline 10351792 & 27.00 & 3.60 & 27.63 & 3.52 & 27.04 & 3.74 & 26.20 & 3.60 & 26.91 & & 27.81 & 29.12 & 3.60 \\
\hline 10353044 & 33.60 & 4.14 & 33.87 & 4.18 & 33.32 & 4.22 & 33.70 & 4.15 & 31.33 & 4.21 & 34.16 & 35.08 & 4.16 \\
\hline 10382615 & 30.50 & 4.14 & 29.24 & 4.04 & & & 31.02 & & & & 30.37 & 31.33 & \\
\hline 10383488 & 14.10 & 1.98 & & & 13.87 & 1.97 & 14.38 & 1.99 & & & & & \\
\hline 10383668 & 55.30 & 4.95 & 55.21 & & 55.02 & 4.83 & 55.84 & 5.02 & 54.89 & & 55.76 & 57.81 & 4.96 \\
\hline 10384255 & 43.40 & 4.52 & 43.02 & 3.67 & 43.67 & 4.37 & 43.95 & & & & 43.34 & 44.63 & \\
\hline 10386904 & 178.30 & 14.63 & 179.15 & 14.79 & 178.14 & 14.67 & 177.91 & 14.81 & & & & & 14.61 \\
\hline 10388249 & 52.50 & 5.50 & 52.76 & 5.42 & 52.58 & 5.40 & 52.53 & 5.41 & 52.95 & 5.89 & 52.19 & 53.97 & 5.52 \\
\hline 10389037 & & & 39.66 & 4.13 & & & & & & & 40.12 & 41.24 & 4.04 \\
\hline 10389393 & 37.80 & 4.32 & 38.71 & 4.44 & 37.65 & 4.26 & 38.80 & 4.45 & & & 39.22 & 41.13 & 4.31 \\
\hline 10396693 & & & & & & & & & 1.10 & 0.25 & & & \\
\hline 10403036 & 10.10 & 1.42 & & & & & 10.32 & & 8.99 & 1.49 & & & \\
\hline 10403323 & 28.80 & 3.68 & 29.00 & 3.68 & & & 28.83 & & & & 29.48 & 30.85 & 3.71 \\
\hline 10404994 & 41.10 & 4.42 & & & & & & & & & & & \\
\hline 10405285 & 19.50 & 2.64 & 18.95 & 2.56 & 18.82 & 2.72 & 20.09 & 2.53 & & & 19.37 & 20.11 & \\
\hline 10418503 & 9.50 & 1.57 & & & & & 9.73 & & & & & & \\
\hline 10426331 & 18.80 & 2.59 & 18.95 & 2.60 & 19.24 & 2.59 & 18.80 & 2.59 & 15.43 & & 19.01 & 20.35 & 2.61 \\
\hline 10426854 & 40.50 & 4.24 & & & 39.41 & 4.91 & 40.41 & 4.40 & 36.52 & 4.05 & 40.12 & 40.63 & 4.29 \\
\hline 10456555 & 51.60 & 4.77 & 50.17 & 4.85 & 51.49 & 4.79 & 50.73 & 4.84 & 49.83 & & 52.20 & 56.57 & 4.80 \\
\hline 10463137 & 30.00 & 4.00 & 29.61 & 4.12 & & & 29.91 & & & & 30.13 & 31.63 & 4.04 \\
\hline 10467570 & 28.60 & 4.07 & & & & & 29.94 & & & & & & \\
\hline 10468528 & 26.60 & 3.68 & 26.63 & 3.66 & 26.46 & 3.66 & 27.73 & & 25.43 & 3.68 & 26.99 & 28.52 & 3.71 \\
\hline 10474071 & 84.30 & 7.47 & 85.40 & 7.64 & 87.80 & 7.41 & 83.28 & 7.51 & & & 88.77 & 98.01 & 7.52 \\
\hline 10483504 & 105.30 & 8.70 & 103.79 & 8.61 & 104.42 & 8.67 & 104.86 & 8.67 & & & 104.47 & 107.44 & 8.77 \\
\hline $\begin{array}{l}10489073 \\
10514458\end{array}$ & 33.40 & 4.07 & 33.82 & 4.13 & 33.81 & 4.13 & $\begin{array}{l}33.65 \\
10.59\end{array}$ & 4.03 & & & 33.97 & 36.15 & 4.02 \\
\hline $\begin{array}{l}10514458 \\
10527754\end{array}$ & 59.70 & 6.08 & 59.99 & 6.00 & 59.64 & 5.97 & 59.70 & 6.00 & & & 59.87 & 61.86 & 6.01 \\
\hline 10533369 & 42.30 & 4.24 & 41.17 & 4.24 & 39.78 & & 42.95 & & & & 42.29 & 41.94 & 4.23 \\
\hline 10552202 & 38.30 & 4.30 & 37.75 & 4.48 & 37.44 & 4.31 & 38.52 & 4.34 & & & 38.77 & 39.34 & 4.32 \\
\hline 10582841 & 28.50 & 3.35 & & & & & 28.66 & & 27.55 & 3.79 & 29.02 & 30.21 & 3.00 \\
\hline 10590683 & 26.30 & 3.76 & 26.06 & 3.77 & & & 27.34 & 3.70 & & & 26.53 & 27.78 & 3.71 \\
\hline 10592249 & 38.20 & 4.24 & 38.29 & 4.21 & 38.57 & 3.89 & 38.78 & 4.22 & & & 38.73 & 38.71 & 4.25 \\
\hline 10593078 & 197.50 & 15.28 & 204.96 & 15.34 & 200.00 & 15.48 & 204.06 & 15.41 & & & 203.56 & 193.92 & 15.43 \\
\hline 10600926 & 29.50 & 4.17 & 30.03 & 4.18 & 28.69 & 3.85 & 31.17 & & & & 31.26 & 31.10 & 4.12 \\
\hline 10602374 & 34.80 & 4.09 & 35.85 & 4.10 & 35.77 & 4.44 & 35.21 & & & & & & \\
\hline 10602976 & & & & & & & 36.96 & & & & & & \\
\hline 10604460 & 30.20 & 3.80 & 30.44 & 3.90 & 29.92 & 3.77 & 30.59 & 3.75 & 27.09 & 3.80 & 30.70 & 30.52 & 3.75 \\
\hline
\end{tabular}


Table 7. continued.

\begin{tabular}{|c|c|c|c|c|c|c|c|c|c|c|c|c|c|}
\hline Star & $\begin{array}{l}\text { COR } \\
v_{\max } \\
\mu \mathrm{Hz}\end{array}$ & $\begin{array}{c}\mathrm{COR} \\
\Delta v \\
\mu \mathrm{Hz}\end{array}$ & $\begin{array}{l}\text { CAN } \\
v_{\max } \\
\mu \mathrm{Hz}\end{array}$ & $\begin{array}{c}\mathrm{CAN} \\
\Delta v \\
\mu \mathrm{Hz}\end{array}$ & $\begin{array}{l}\mathrm{A} 2 \mathrm{Z} \\
v_{\max } \\
\mu \mathrm{Hz}\end{array}$ & $\begin{array}{c}\mathrm{A} 2 \mathrm{Z} \\
\Delta v \\
\mu \mathrm{Hz}\end{array}$ & $\begin{array}{l}\text { SYD } \\
v_{\max } \\
\mu \mathrm{Hz}\end{array}$ & $\begin{array}{c}\text { SYD } \\
\Delta v \\
\mu \mathrm{Hz}\end{array}$ & $\begin{array}{l}\text { DLB } \\
v_{\max } \\
\mu \mathrm{Hz}\end{array}$ & $\begin{array}{c}\overline{\mathrm{DLB}} \\
\Delta v \\
\mu \mathrm{Hz}\end{array}$ & $\begin{array}{c}\text { OCT I } \\
v_{\max } \\
\mu \mathrm{Hz}\end{array}$ & $\begin{array}{c}\text { OCT II } \\
v_{\max } \\
\mu \mathrm{Hz}\end{array}$ & $\begin{array}{c}\mathrm{OCT} \\
\Delta v \\
\mu \mathrm{Hz}\end{array}$ \\
\hline 10614707 & 34.30 & 4.17 & 34.39 & 4.36 & 34.20 & 4.61 & 35.21 & 4.21 & 36.43 & 4.17 & 34.79 & 36.26 & 4.16 \\
\hline 10619191 & 40.70 & 4.46 & 41.60 & 4.37 & 40.63 & 4.51 & 40.83 & 4.37 & & & 41.70 & 43.72 & 4.47 \\
\hline 10647243 & 41.80 & 4.22 & 39.17 & 4.27 & & & 42.21 & & & & 39.42 & 40.75 & 4.18 \\
\hline 10649021 & & & & & & & & & 21.77 & 3.05 & & & \\
\hline 10649096 & 25.90 & 3.39 & & & & & 26.13 & & 25.54 & 3.38 & 26.08 & 27.17 & \\
\hline 10651578 & 31.60 & 4.00 & 31.00 & 3.80 & & & 31.62 & & & & 32.03 & 33.29 & 4.02 \\
\hline 10656124 & 10.40 & 1.57 & & & & & 10.42 & & 10.58 & 1.38 & & & \\
\hline 10659688 & 23.20 & 3.18 & 23.32 & 3.19 & 22.36 & 3.17 & 23.03 & 3.18 & & & 23.59 & 24.42 & 3.21 \\
\hline 10662618 & 31.50 & 3.93 & 31.24 & 4.07 & 31.06 & 3.87 & 32.42 & 4.01 & 25.93 & 3.47 & & & \\
\hline 10669876 & 19.50 & 2.50 & & & 19.61 & 2.39 & 19.69 & 2.57 & & & 19.35 & 19.22 & \\
\hline 10677958 & 9.10 & 1.43 & & & 9.25 & 1.27 & 9.38 & & 9.95 & 1.81 & & & \\
\hline 10709799 & 36.30 & 4.19 & 36.89 & 4.16 & 36.18 & 4.15 & 36.55 & 4.19 & 32.81 & 4.67 & 36.68 & 38.21 & 4.10 \\
\hline 10711145 & 34.10 & 4.24 & 33.78 & 4.15 & 33.54 & 4.08 & 34.69 & 4.16 & & & 34.27 & 34.39 & 4.19 \\
\hline 10711154 & 46.50 & 4.74 & 46.11 & 4.71 & 46.33 & 4.67 & 47.24 & 4.70 & & & 46.49 & 48.56 & 4.70 \\
\hline 10716853 & 45.00 & 4.95 & & & & & & & & & & & \\
\hline 10721900 & 39.90 & 4.02 & 37.13 & 4.06 & 37.02 & 3.97 & 40.16 & 3.96 & & & 37.55 & 38.25 & \\
\hline 10724027 & 31.80 & 4.09 & 31.62 & 4.02 & 31.88 & 4.05 & 32.66 & 4.01 & 29.01 & 4.30 & 31.84 & 32.28 & \\
\hline 10743092 & 19.50 & 2.40 & 19.76 & 2.52 & & & 19.62 & 2.57 & & & 19.61 & 19.92 & 2.37 \\
\hline 10743515 & 44.20 & 4.46 & 44.83 & 4.57 & 44.54 & 4.55 & 44.54 & 4.52 & & & 45.02 & 46.59 & 4.46 \\
\hline 10747240 & 34.60 & 3.97 & 34.04 & 4.14 & & & 33.98 & & & & 34.70 & 36.15 & 3.91 \\
\hline 10749112 & 47.00 & 5.17 & 47.51 & 5.20 & 47.22 & 5.00 & 47.08 & 5.20 & & & 47.61 & 49.30 & 5.11 \\
\hline 10776256 & & & & & & & 183.26 & 13.21 & & & & & \\
\hline 10777816 & 201.70 & 15.62 & 210.43 & 15.73 & 210.41 & 15.48 & 209.72 & 15.73 & & & & & 15.63 \\
\hline 10778969 & 36.60 & 4.19 & 37.39 & 4.30 & 36.53 & 4.15 & 36.62 & 4.28 & & & 37.45 & 38.48 & 4.22 \\
\hline 10779177 & & & & & & & 231.15 & 17.20 & & & & & \\
\hline 10784013 & 15.40 & 2.46 & 15.55 & 2.46 & & & 15.36 & & & & & & \\
\hline 10788228 & 37.90 & 4.22 & & & & & & & & & & & \\
\hline 10796378 & 40.50 & 4.74 & 40.74 & 4.70 & 41.16 & 4.78 & 40.83 & 4.72 & & & 41.44 & 41.77 & 4.69 \\
\hline 10799530 & 36.90 & 4.27 & 37.12 & 4.32 & & & 36.92 & & & & 37.81 & 39.01 & 4.22 \\
\hline 10816748 & 32.60 & 3.86 & 31.90 & 3.79 & 32.28 & 3.84 & 32.78 & 3.85 & & & 32.78 & 34.02 & \\
\hline 10842231 & 37.90 & 3.95 & 37.70 & 4.25 & & & 38.48 & 4.24 & 32.65 & & 38.33 & 40.60 & 4.22 \\
\hline 10847321 & 69.50 & 6.49 & 69.23 & 6.54 & 68.74 & 6.51 & 69.43 & 6.59 & & & 69.42 & 71.18 & 6.45 \\
\hline 10849541 & 20.00 & 2.83 & 20.65 & 2.83 & 19.64 & 2.74 & 20.12 & 2.87 & & & 20.50 & 21.95 & \\
\hline 10858331 & 28.50 & 4.02 & 27.84 & 3.99 & & & 28.40 & 4.02 & & & 28.69 & 29.70 & 3.95 \\
\hline 10858520 & 33.40 & 3.82 & 34.38 & 3.82 & 33.13 & 3.71 & 33.56 & 3.81 & & & 34.59 & 35.89 & 3.80 \\
\hline 10859779 & 81.80 & 7.39 & 82.10 & 7.38 & 81.77 & 7.50 & 81.36 & 7.29 & & & 82.50 & 81.18 & 7.40 \\
\hline 10863535 & 8.20 & 1.29 & & & & & 8.19 & & & & & & \\
\hline 10864711 & 44.10 & 4.95 & 43.43 & 4.89 & & & 44.17 & 4.90 & 41.98 & 4.84 & 44.00 & 45.35 & 4.91 \\
\hline 10866415 & 96.50 & 8.81 & 96.33 & 8.70 & 96.39 & 8.97 & 96.40 & 8.72 & 97.51 & & 95.73 & 97.27 & 8.76 \\
\hline 10903016 & 24.30 & 3.18 & 24.23 & 3.25 & 24.18 & & 24.67 & 3.28 & & & 24.51 & 25.75 & 3.12 \\
\hline 10903291 & 33.50 & 3.97 & 32.92 & 3.98 & 32.79 & 3.56 & 33.62 & 3.99 & & & 33.54 & 35.41 & 4.01 \\
\hline 10907196 & 44.10 & 4.74 & 44.84 & 4.74 & 43.60 & 4.81 & 43.52 & 4.63 & & & 44.94 & 44.95 & 4.72 \\
\hline 10910802 & 22.90 & 3.12 & 23.13 & 3.26 & & & 22.66 & 3.16 & & & & & \\
\hline 10910889 & 46.70 & 4.61 & 44.66 & 4.83 & 44.02 & 4.66 & 48.20 & & & & 46.25 & 46.70 & 4.61 \\
\hline 10918731 & 43.80 & 4.61 & 43.56 & 4.91 & 44.59 & 4.65 & 43.84 & 4.55 & & & 44.88 & 46.50 & 4.59 \\
\hline 10922821 & 33.00 & 3.76 & 33.75 & 4.04 & 32.71 & 3.91 & 32.86 & 3.54 & & & 33.76 & 35.09 & \\
\hline 10933384 & 9.30 & 1.60 & & & & & 9.58 & 1.56 & & & & & \\
\hline 10961390 & 31.70 & 3.93 & 32.54 & 4.08 & 31.89 & & 31.79 & 4.01 & & & 32.51 & 34.62 & 3.95 \\
\hline 10964584 & 30.60 & 3.95 & 30.79 & 3.43 & & & 30.63 & & & & 30.92 & 32.23 & \\
\hline 10965536 & 25.30 & 3.27 & 25.35 & 3.30 & 25.24 & & 26.23 & & & & 26.17 & 25.65 & 3.21 \\
\hline 10969616 & 29.80 & 3.82 & & & & & 31.20 & 3.76 & & & & & \\
\hline 10973854 & 32.60 & 3.62 & 32.42 & 3.61 & 32.24 & 3.73 & 32.93 & 3.55 & 31.04 & 3.91 & 32.50 & 32.92 & 3.62 \\
\hline 10977629 & 24.20 & 3.26 & 24.64 & 3.28 & 23.89 & 3.19 & 24.50 & 3.23 & & & 24.75 & 25.53 & 3.22 \\
\hline 10979181 & & & & & & & 9.65 & & & & & & \\
\hline 10992126 & 7.60 & 1.34 & & & & & 7.77 & & 7.80 & 1.25 & & & \\
\hline 11018481 & & & & & & & 10.44 & & & & & & \\
\hline 11018745 & 32.80 & 4.09 & 33.46 & 4.42 & 32.57 & 4.02 & 32.88 & 4.06 & & & 34.29 & 36.08 & 4.06 \\
\hline 11018922 & 31.70 & 4.07 & 31.53 & 4.07 & 31.47 & 4.05 & 32.52 & 3.96 & & & 32.48 & 34.27 & 4.01 \\
\hline 11019452 & & & & & & & 192.35 & & & & & & \\
\hline 11027708 & & & & & & & & & 18.37 & 2.93 & & & \\
\hline 11028372 & 37.80 & 4.68 & 38.79 & 4.62 & 37.93 & 4.54 & 37.78 & 4.61 & & & 38.53 & 40.93 & \\
\hline 11028575 & 34.70 & 4.35 & 35.68 & 4.54 & & & 34.42 & 4.32 & & & 35.44 & 38.58 & \\
\hline 11037292 & 16.70 & 2.45 & 16.87 & 2.39 & & & 16.73 & 2.42 & & & 16.89 & 17.98 & \\
\hline 11042388 & 8.50 & 1.46 & & & 8.90 & 1.47 & 8.68 & 1.47 & & & & & \\
\hline 11043208 & 51.40 & 5.41 & 52.34 & 5.35 & 51.49 & 5.41 & 51.37 & 5.40 & & & 52.23 & 52.48 & 5.44 \\
\hline
\end{tabular}


S. Hekker et al.: Comparison of global oscillation parameters from different methods

Table 7. continued.

\begin{tabular}{|c|c|c|c|c|c|c|c|c|c|c|c|c|c|}
\hline Star & $\begin{array}{l}\mathrm{COR} \\
v_{\max } \\
\mu \mathrm{Hz} \\
\end{array}$ & $\begin{array}{c}\text { COR } \\
\Delta v \\
\mu \mathrm{Hz}\end{array}$ & $\begin{array}{l}\text { CAN } \\
\nu_{\max } \\
\mu \mathrm{Hz} \\
\end{array}$ & $\begin{array}{c}\text { CAN } \\
\Delta v \\
\mu \mathrm{Hz}\end{array}$ & $\begin{array}{l}\mathrm{A} 2 \mathrm{Z} \\
v_{\max } \\
\mu \mathrm{Hz}\end{array}$ & $\begin{array}{c}\mathrm{A} 2 \mathrm{Z} \\
\Delta v \\
\mu \mathrm{Hz}\end{array}$ & $\begin{array}{l}\text { SYD } \\
v_{\max } \\
\mu \mathrm{Hz}\end{array}$ & $\begin{array}{c}\text { SYD } \\
\Delta v \\
\mu \mathrm{Hz}\end{array}$ & $\begin{array}{c}\text { DLB } \\
v_{\max } \\
\mu \mathrm{Hz}\end{array}$ & $\begin{array}{c}\text { DLB } \\
\Delta v \\
\mu \mathrm{Hz}\end{array}$ & $\begin{array}{c}\text { OCT I } \\
v_{\max } \\
\mu \mathrm{Hz}\end{array}$ & $\begin{array}{c}\text { OCT II } \\
v_{\max } \\
\mu \mathrm{Hz}\end{array}$ & $\begin{array}{c}\mathrm{OCT} \\
\Delta v \\
\mu \mathrm{Hz}\end{array}$ \\
\hline 11043832 & 58.20 & 5.68 & 58.69 & 5.70 & 57.85 & 5.71 & 57.98 & 5.69 & 53.62 & & 58.99 & 63.03 & 5.69 \\
\hline 11045542 & 7.50 & 1.30 & & & 7.52 & 1.02 & 7.64 & & 8.09 & 1.45 & & & \\
\hline 11046496 & 31.30 & 3.80 & 31.76 & 3.74 & 31.50 & 3.89 & 31.40 & 3.82 & 27.18 & 3.75 & & & \\
\hline 11072852 & 9.70 & 1.48 & & & & & 9.78 & & 8.44 & 1.18 & & & \\
\hline 11075908 & 37.20 & 4.24 & 37.29 & 4.46 & 37.53 & 4.20 & 37.56 & 4.19 & & & 37.99 & 39.28 & 4.23 \\
\hline 11081838 & 30.30 & 3.78 & 30.51 & 3.91 & & & 31.11 & & & & 31.32 & 32.41 & \\
\hline 11083701 & 31.90 & 3.97 & 31.75 & 3.94 & & & 32.34 & 3.90 & & & 32.25 & 33.91 & 4.05 \\
\hline 11084022 & 43.90 & 4.61 & 44.58 & 4.68 & 44.02 & 4.62 & 43.97 & 4.70 & & & 44.69 & 47.07 & 4.61 \\
\hline 11126721 & 29.80 & 3.54 & 29.24 & 3.54 & 28.59 & 3.50 & 30.26 & & & & 29.77 & 30.17 & 3.50 \\
\hline 11128910 & 35.30 & 4.19 & 35.09 & 4.23 & 35.29 & 4.15 & 35.97 & 4.13 & 32.43 & 4.53 & 35.82 & 37.42 & 4.16 \\
\hline 11129153 & 26.90 & 3.68 & & & 25.73 & 3.64 & 28.78 & & & & & & \\
\hline 11134608 & 8.40 & 1.29 & & & & & 8.54 & & & & & & \\
\hline 11138375 & 34.20 & 3.93 & 34.76 & 3.94 & & & 34.96 & & & & 35.06 & 35.88 & 3.89 \\
\hline 11153989 & 37.80 & 4.14 & 37.35 & 4.04 & 36.85 & 4.15 & 38.03 & 4.06 & & & 38.33 & 40.07 & 4.09 \\
\hline 11177727 & 31.50 & 3.88 & 31.75 & 3.89 & 31.30 & 3.87 & 32.19 & 3.78 & & & 32.24 & 33.24 & 3.84 \\
\hline 11177729 & & & & & 35.71 & 3.55 & 35.88 & & & & & & \\
\hline 11177749 & 33.70 & 4.09 & 33.62 & 4.13 & 33.51 & 3.99 & 33.67 & 4.12 & & & 33.85 & 36.01 & 4.07 \\
\hline 11178396 & & & & & 26.33 & 2.38 & 25.81 & & & & & & \\
\hline 11188067 & 38.10 & 4.27 & 38.08 & 4.29 & 38.52 & 4.14 & 38.48 & 4.19 & 34.73 & & 38.72 & 39.25 & 4.29 \\
\hline 11190322 & 55.00 & 5.50 & 55.09 & 5.45 & 54.86 & 5.45 & 55.06 & 5.48 & & & 55.02 & 54.82 & 5.50 \\
\hline 11199105 & 30.00 & 3.91 & 30.12 & 4.10 & & & 30.06 & 3.81 & & & 30.60 & 32.39 & 3.92 \\
\hline 11206069 & 13.50 & 1.64 & & & & & 13.61 & 1.65 & & & & & \\
\hline 11228759 & 35.80 & 4.07 & 36.01 & 4.07 & 35.40 & 4.22 & 36.08 & 4.07 & & & 36.35 & 38.40 & 4.04 \\
\hline 11231549 & 29.70 & 3.47 & 29.65 & 3.55 & 29.31 & 3.48 & 30.16 & 3.49 & 26.85 & 3.62 & 30.26 & 31.75 & \\
\hline 11236536 & 43.00 & 4.44 & 41.04 & 4.60 & 42.50 & 4.59 & 43.15 & 4.60 & 43.03 & 4.02 & 42.69 & 44.50 & 4.44 \\
\hline 11243761 & 9.80 & 1.58 & & & 10.65 & 1.55 & 10.10 & & & & & & \\
\hline 11245234 & 32.60 & 4.32 & 31.86 & 4.32 & 32.58 & 4.48 & 33.23 & & & & 33.27 & 33.17 & \\
\hline 11247049 & 33.80 & 3.86 & & & 33.22 & 4.23 & 33.68 & 3.89 & 29.93 & & 34.27 & 35.92 & 3.82 \\
\hline 11250152 & 30.90 & 3.58 & 30.75 & 3.77 & 30.53 & 3.54 & 31.28 & 3.59 & & & 30.75 & 31.08 & 3.54 \\
\hline 11251115 & 57.50 & 5.09 & 53.87 & 5.24 & 55.32 & 5.16 & 58.55 & 5.02 & 44.10 & 5.20 & 56.90 & 61.23 & 5.10 \\
\hline 11252233 & 32.70 & 3.93 & 31.94 & 3.70 & 31.56 & 3.94 & 33.28 & & & & 32.62 & 33.69 & 3.98 \\
\hline 11252706 & 41.00 & 4.55 & 41.27 & & & & 40.58 & & & & 41.99 & 43.60 & 4.52 \\
\hline 11282383 & 74.50 & 7.16 & 75.26 & 7.23 & 74.78 & 7.33 & 74.52 & 7.23 & & & 74.61 & 77.74 & 7.13 \\
\hline 11284760 & 34.10 & 4.41 & 33.28 & 4.22 & & & 34.37 & & & & 34.67 & 36.67 & 4.18 \\
\hline 11284798 & 10.10 & 1.43 & & & & & 10.44 & & 9.73 & & & & \\
\hline 11289335 & 35.80 & 4.35 & 36.75 & 4.14 & & & 35.53 & & & & 36.94 & 38.20 & 4.16 \\
\hline 11296211 & 33.50 & 3.88 & 33.06 & 4.08 & 33.27 & 3.95 & 33.48 & 4.01 & & & 33.66 & 34.19 & 3.88 \\
\hline 11308784 & 61.90 & 6.19 & 61.87 & 6.11 & 61.79 & 6.25 & 61.95 & 6.16 & & & 62.31 & 63.04 & 6.18 \\
\hline 11338267 & 25.30 & 3.30 & 25.33 & 3.31 & & & 25.24 & & & & 25.39 & 26.47 & \\
\hline 11339000 & 37.30 & 4.19 & 38.71 & 4.39 & 37.83 & 4.22 & 38.73 & 4.30 & & & 39.11 & 40.98 & 4.18 \\
\hline 11340165 & 34.90 & 4.07 & 33.90 & 4.56 & 34.08 & 4.12 & 35.24 & 4.10 & & & 34.62 & 35.11 & 4.14 \\
\hline 11342694 & 75.10 & 7.01 & 75.33 & 7.12 & 75.34 & 7.06 & 75.52 & 7.13 & 75.26 & & 75.45 & 75.75 & 6.96 \\
\hline 11350954 & 21.30 & 2.40 & & & & & 21.50 & 2.41 & & & 21.65 & 22.37 & 2.45 \\
\hline 11352756 & 26.70 & 3.82 & & & 26.45 & 3.64 & 25.78 & & 26.35 & 2.63 & & & \\
\hline 11353313 & 113.90 & 10.11 & 124.90 & 10.79 & 128.71 & 10.55 & 128.26 & 10.75 & & & 125.95 & 123.63 & 10.70 \\
\hline $\begin{array}{l}11359175 \\
11394497\end{array}$ & 33.90 & 4.27 & 34.70 & 4.65 & & & $\begin{array}{c}33.77 \\
6.06\end{array}$ & & & & 34.99 & 37.20 & 4.24 \\
\hline 11403437 & 34.20 & 4.24 & 33.26 & 4.24 & 34.64 & 4.24 & 35.58 & 4.19 & & & 34.94 & 35.85 & 4.22 \\
\hline 11409529 & 36.50 & 4.00 & 34.64 & 4.00 & 34.77 & 3.96 & 36.22 & 3.97 & & & 35.62 & 36.65 & 4.01 \\
\hline 11410549 & 37.00 & 4.22 & 37.34 & 4.28 & 37.08 & & 37.80 & & 35.21 & 4.76 & 37.64 & 38.90 & 4.21 \\
\hline 11444313 & 33.20 & 3.88 & 32.52 & 3.93 & 33.04 & 3.91 & 33.47 & 3.52 & 30.45 & 3.53 & 33.05 & 33.59 & 3.89 \\
\hline 11455176 & 37.80 & 4.17 & 36.76 & 4.18 & & & 37.42 & & & & 37.02 & 38.34 & \\
\hline 11456449 & 113.60 & 9.29 & 111.83 & 9.34 & 111.10 & 9.23 & 110.88 & 9.37 & & & 112.43 & 113.93 & 9.25 \\
\hline 11465784 & 35.70 & 4.24 & 34.50 & 4.25 & 34.35 & 4.39 & 36.70 & & & & 35.90 & 37.05 & 4.25 \\
\hline 11469401 & 29.00 & 3.70 & 30.47 & 3.63 & & & 29.29 & & & & 31.16 & 32.61 & 3.69 \\
\hline 11494511 & 12.40 & 1.78 & & & & & 12.79 & 1.77 & & & & & \\
\hline 11499428 & 32.10 & 3.97 & 32.22 & 4.09 & 31.54 & 4.01 & 32.79 & 3.98 & & & 33.15 & 34.24 & 3.99 \\
\hline 11502218 & 23.20 & 2.76 & & & & & & & & & & & \\
\hline 11509277 & 44.10 & 4.71 & 43.18 & 4.78 & 43.76 & 4.75 & 43.92 & 4.73 & 41.06 & & 44.84 & 47.32 & 4.67 \\
\hline 11509792 & 19.10 & 2.77 & 19.70 & 2.82 & 18.41 & 2.72 & 19.33 & 2.79 & 18.74 & 2.39 & & & \\
\hline 11518492 & 52.40 & 5.33 & 52.25 & 5.36 & 52.08 & 5.28 & 52.87 & 5.36 & 50.47 & 5.21 & 52.46 & 53.48 & 5.28 \\
\hline 11521352 & 106.00 & 9.17 & 106.31 & 9.15 & 106.15 & 9.32 & 105.36 & 9.20 & & & 106.85 & 108.41 & 9.25 \\
\hline 11546335 & & & & & & & & & 91.23 & 8.15 & & & \\
\hline 11548940 & 23.80 & 3.10 & 23.37 & 3.01 & 21.82 & 2.44 & 25.07 & & & & & & \\
\hline 11550492 & 93.60 & 8.59 & 93.56 & 8.63 & 93.74 & 8.80 & 93.61 & 8.60 & 90.30 & & 93.16 & 95.55 & 8.57 \\
\hline
\end{tabular}


Table 7. continued.

\begin{tabular}{|c|c|c|c|c|c|c|c|c|c|c|c|c|c|}
\hline Star & $\begin{array}{c}\text { COR } \\
v_{\max } \\
\mu \mathrm{Hz}\end{array}$ & $\begin{array}{c}\text { COR } \\
\Delta v \\
\mu \mathrm{Hz}\end{array}$ & $\begin{array}{l}\text { CAN } \\
v_{\max } \\
\mu \mathrm{Hz}\end{array}$ & $\begin{array}{c}\text { CAN } \\
\Delta v \\
\mu \mathrm{Hz}\end{array}$ & $\begin{array}{l}\mathrm{A} 2 \mathrm{Z} \\
v_{\max } \\
\mu \mathrm{Hz}\end{array}$ & $\begin{array}{c}\mathrm{A} 2 \mathrm{Z} \\
\Delta v \\
\mu \mathrm{Hz}\end{array}$ & $\begin{array}{l}\text { SYD } \\
v_{\max } \\
\mu \mathrm{Hz}\end{array}$ & $\begin{array}{c}\text { SYD } \\
\Delta v \\
\mu \mathrm{Hz}\end{array}$ & $\begin{array}{c}\text { DLB } \\
v_{\max } \\
\mu \mathrm{Hz}\end{array}$ & $\begin{array}{c}\text { DLB } \\
\Delta v \\
\mu \mathrm{Hz}\end{array}$ & $\begin{array}{c}\text { OCT I } \\
v_{\max } \\
\mu \mathrm{Hz}\end{array}$ & $\begin{array}{c}\text { OCT II } \\
v_{\max } \\
\mu \mathrm{Hz}\end{array}$ & $\begin{array}{c}\mathrm{OCT} \\
\Delta v \\
\mu \mathrm{Hz}\end{array}$ \\
\hline 11550567 & 39.00 & 4.19 & 37.17 & 4.01 & 37.18 & 4.17 & 39.83 & & & & 37.60 & 38.10 & 4.16 \\
\hline 11551628 & 51.50 & 4.95 & 51.56 & 5.13 & 51.63 & 4.91 & 52.13 & 4.93 & & & 51.89 & 54.16 & 4.97 \\
\hline 11551746 & 45.80 & 4.68 & 44.60 & 4.79 & 45.15 & 4.64 & 46.52 & 4.72 & & & 45.27 & 45.90 & 4.81 \\
\hline 11564787 & 28.00 & 3.64 & 28.77 & 3.63 & & & 28.33 & & & & 29.08 & 30.89 & 3.56 \\
\hline 11568605 & 49.50 & 4.98 & 49.86 & 4.91 & 49.52 & 5.00 & 49.91 & 4.91 & 51.48 & & 50.04 & 50.36 & 4.95 \\
\hline 11569659 & 30.30 & 4.09 & 30.60 & 4.15 & 30.34 & 4.06 & 30.33 & & & & 31.07 & 34.52 & \\
\hline 11598116 & 17.40 & 2.58 & 17.78 & 2.59 & 17.62 & & 17.55 & 2.53 & & & 18.25 & 18.90 & \\
\hline 11599067 & 24.20 & 3.06 & 23.60 & 3.22 & 23.72 & 3.03 & 24.47 & 3.02 & & & 24.05 & 24.73 & 3.03 \\
\hline 11603064 & 15.40 & 1.77 & & & 15.46 & 1.62 & 15.64 & & 14.49 & 2.25 & & & \\
\hline 11606796 & 32.10 & 4.09 & 31.89 & 3.53 & & & 32.61 & & 29.53 & 3.73 & 32.32 & 34.70 & \\
\hline 11612171 & 35.30 & 4.07 & 34.38 & 3.89 & 33.79 & 4.04 & 35.91 & & & & & & \\
\hline 11612320 & 9.00 & 1.44 & & & & & 9.35 & & & & & & \\
\hline 11615224 & 28.50 & 4.02 & 29.30 & 4.03 & 28.67 & 3.94 & 28.63 & 4.04 & 28.32 & 3.76 & 29.74 & 32.26 & 3.96 \\
\hline 11617501 & 43.40 & 4.71 & 43.28 & 4.68 & 43.10 & 4.82 & 43.70 & 4.67 & & & 43.45 & 43.50 & 4.69 \\
\hline 11617622 & 31.30 & 3.88 & 32.07 & 4.01 & 31.70 & 3.87 & 31.33 & 3.84 & & & 32.57 & 34.50 & 3.84 \\
\hline 11617751 & 12.80 & 1.90 & & & 12.60 & 1.74 & 13.44 & & 13.87 & & & & \\
\hline 11618103 & 106.60 & 9.42 & 106.37 & 9.41 & 107.16 & 9.31 & 106.92 & 9.38 & 100.68 & 8.89 & 107.02 & 107.37 & 9.38 \\
\hline 11649869 & 16.80 & 2.66 & & & & & 16.87 & & & & & & \\
\hline 11653073 & 39.00 & 4.27 & 38.34 & 4.28 & 38.49 & 4.06 & 39.59 & 4.08 & 36.04 & 3.87 & 38.75 & 40.06 & 4.30 \\
\hline 11656670 & 14.00 & 1.99 & & & 14.39 & 2.03 & 14.63 & 1.97 & 14.02 & 1.67 & & & \\
\hline 11657684 & 33.00 & 3.91 & 33.57 & 4.20 & & & 33.06 & & & & 33.93 & 35.21 & 3.99 \\
\hline 11658270 & 25.30 & 3.10 & 24.65 & 3.11 & 24.63 & 3.01 & 25.49 & 3.10 & & & 25.34 & 26.26 & 3.07 \\
\hline 11658849 & 29.90 & 4.07 & 30.37 & 3.71 & & & 30.43 & 3.75 & & & & & \\
\hline 11668246 & 38.10 & 4.24 & 39.02 & 4.31 & 38.96 & 4.26 & 38.54 & 4.23 & & & 40.06 & 41.53 & 4.27 \\
\hline 11670252 & 4.90 & 0.83 & & & & & & & & & & & \\
\hline 11673900 & 43.90 & 4.64 & 43.74 & 4.68 & 44.28 & 4.62 & 43.84 & 4.59 & & & 44.10 & 46.50 & 4.71 \\
\hline 11674666 & 32.00 & 4.07 & 31.33 & 4.04 & 31.11 & & 32.27 & 4.01 & & & 32.21 & 33.62 & 3.99 \\
\hline 11674677 & 37.30 & 3.82 & 36.88 & 3.97 & 35.88 & 3.68 & 37.76 & & & & 37.71 & 37.58 & 3.83 \\
\hline 11704163 & 46.80 & 4.81 & 46.98 & 4.77 & 46.23 & 4.82 & 47.46 & 4.82 & 42.21 & 4.55 & 47.48 & 48.46 & 4.78 \\
\hline 11706564 & & & 21.06 & 2.67 & & & & & & & 21.20 & 22.06 & 2.78 \\
\hline 11707304 & 35.30 & 4.07 & 34.97 & 4.15 & 34.89 & 4.08 & 35.61 & 4.11 & & & 35.11 & 36.18 & 4.07 \\
\hline 11707798 & 38.40 & 4.19 & 38.77 & 4.29 & 38.12 & 4.20 & 38.33 & 4.38 & 33.65 & & 38.52 & 40.05 & 4.22 \\
\hline 11721438 & 111.10 & 8.70 & 109.34 & 8.58 & & & 110.14 & 8.60 & & & 111.02 & 113.30 & 8.62 \\
\hline 11752358 & 63.00 & 6.61 & 62.98 & 6.60 & 63.05 & 6.72 & 63.25 & 6.57 & & & 63.19 & 65.31 & 6.58 \\
\hline 11752707 & 20.30 & 2.59 & 20.55 & 2.61 & 19.99 & 2.60 & 20.27 & 2.60 & & & 20.66 & 21.75 & 2.61 \\
\hline 11753010 & 15.40 & 2.03 & & & 15.55 & 2.03 & & & 15.41 & 2.18 & & & \\
\hline 11753722 & 46.90 & 5.25 & 47.09 & 5.31 & 46.03 & 5.20 & 47.56 & 5.32 & & & 47.54 & 47.98 & 5.16 \\
\hline 11754056 & 33.90 & 4.24 & 34.25 & 4.30 & 33.48 & 4.01 & 33.01 & 4.15 & & & 34.61 & 35.94 & 4.16 \\
\hline 11757739 & 35.70 & 4.55 & 35.36 & 4.39 & 35.25 & 4.57 & 35.70 & & 31.90 & 4.54 & 36.00 & 38.67 & 4.59 \\
\hline 11758710 & 54.60 & 5.13 & 52.86 & 5.19 & 52.65 & 5.12 & 54.70 & 5.20 & & & 53.51 & 56.31 & 5.07 \\
\hline 11770790 & 10.30 & 1.90 & & & & & 11.90 & & 12.00 & 2.03 & & & \\
\hline 11773146 & 3.90 & 0.67 & & & & & & & & & & & \\
\hline 11775000 & 6.90 & 1.05 & & & & & 7.01 & & & & & & \\
\hline 11805460 & 36.80 & 4.30 & 37.68 & 4.29 & & & 37.11 & 4.28 & 37.97 & 4.04 & 37.59 & 39.19 & 4.45 \\
\hline 11805792 & 33.90 & 4.14 & 33.96 & 4.17 & 33.94 & 4.15 & 34.45 & 4.36 & & & 34.59 & 36.13 & 4.13 \\
\hline 11807840 & 16.40 & 2.18 & 16.82 & 2.26 & & & 16.61 & 2.24 & 13.18 & 2.09 & & & \\
\hline 11807844 & 8.90 & 1.89 & & & & & 9.03 & & & & & & \\
\hline 11817405 & 33.40 & 3.82 & 32.89 & 3.84 & 32.98 & 4.04 & 33.68 & 3.79 & & & 33.09 & 33.86 & 3.79 \\
\hline 11819760 & 31.90 & 3.58 & & & 29.90 & 3.38 & 31.84 & & 29.55 & 3.20 & 31.20 & 31.96 & 3.58 \\
\hline 11821439 & 48.10 & 4.81 & 47.88 & 4.80 & 47.81 & 4.84 & 48.52 & 4.82 & 48.50 & 4.41 & 48.37 & 50.09 & 4.80 \\
\hline 11824403 & 3.10 & 0.71 & & & & & & & & & & & \\
\hline 11854450 & 30.20 & 4.09 & 30.19 & 4.44 & & & 31.38 & 3.94 & & & 30.63 & 30.86 & 3.90 \\
\hline 11854835 & 8.20 & 1.23 & & & & & 8.21 & & & & & & \\
\hline 11855110 & 38.60 & 4.38 & 37.69 & 4.86 & & & 39.37 & & & & 38.55 & 39.23 & 4.39 \\
\hline 11860626 & 41.80 & 4.74 & 42.31 & 4.75 & 41.64 & 4.82 & 41.74 & 4.88 & & & 42.35 & 43.74 & 4.70 \\
\hline 11861823 & 24.10 & 2.98 & 24.39 & 3.04 & 24.23 & 3.00 & 24.80 & 3.01 & & & 24.75 & 25.90 & \\
\hline 11868246 & 33.20 & 3.76 & 33.87 & 4.00 & & & 33.05 & & & & 34.17 & 36.09 & 3.75 \\
\hline 11905840 & 117.70 & 10.11 & 117.09 & 10.33 & 117.60 & 10.45 & 116.37 & 10.31 & & & 117.47 & 123.40 & 10.12 \\
\hline 11909754 & 11.20 & 1.67 & & & & & 11.24 & 1.70 & & & & & \\
\hline 11913545 & 116.10 & 10.11 & 116.73 & 10.07 & 115.73 & 10.00 & 115.38 & 10.10 & & & 116.28 & 122.19 & 10.12 \\
\hline 11960922 & 2.80 & 0.57 & & & & & & & & & & & \\
\hline 11961545 & 48.30 & 5.37 & 48.43 & 5.32 & 48.04 & 5.41 & 48.48 & 5.33 & & & 48.32 & 49.12 & 5.25 \\
\hline 11968334 & 138.00 & 11.27 & 140.43 & 11.29 & 138.56 & 11.36 & 138.20 & 11.28 & & & 140.74 & 143.73 & 11.28 \\
\hline 11973853 & & & & & & & 200.81 & 14.58 & & & & & \\
\hline 12003253 & 32.10 & 3.82 & 31.27 & 3.88 & & & 32.27 & 3.90 & 25.56 & 3.27 & 31.79 & 32.41 & 3.80 \\
\hline
\end{tabular}


S. Hekker et al.: Comparison of global oscillation parameters from different methods

Table 7. continued.

\begin{tabular}{|c|c|c|c|c|c|c|c|c|c|c|c|c|c|}
\hline Star & $\begin{array}{l}\mathrm{COR} \\
v_{\max } \\
\mu \mathrm{Hz}\end{array}$ & $\begin{array}{c}\text { COR } \\
\Delta v \\
\mu \mathrm{Hz}\end{array}$ & $\begin{array}{l}\text { CAN } \\
v_{\max } \\
\mu \mathrm{Hz}\end{array}$ & $\begin{array}{c}\text { CAN } \\
\Delta v \\
\mu \mathrm{Hz}\end{array}$ & $\begin{array}{l}\mathrm{A} 2 \mathrm{Z} \\
v_{\max } \\
\mu \mathrm{Hz}\end{array}$ & $\begin{array}{c}\mathrm{A} 2 \mathrm{Z} \\
\Delta v \\
\mu \mathrm{Hz}\end{array}$ & $\begin{array}{l}\text { SYD } \\
v_{\max } \\
\mu \mathrm{Hz}\end{array}$ & $\begin{array}{c}\text { SYD } \\
\Delta v \\
\mu \mathrm{Hz}\end{array}$ & $\begin{array}{c}\text { DLB } \\
v_{\max } \\
\mu \mathrm{Hz}\end{array}$ & $\begin{array}{c}\text { DLB } \\
\Delta v \\
\mu \mathrm{Hz}\end{array}$ & $\begin{array}{c}\text { OCT I } \\
v_{\max } \\
\mu \mathrm{Hz}\end{array}$ & $\begin{array}{c}\text { OCT II } \\
v_{\max } \\
\mu \mathrm{Hz}\end{array}$ & $\begin{array}{c}\text { OCT } \\
\Delta v \\
\mu \mathrm{Hz}\end{array}$ \\
\hline 12006471 & 38.00 & 4.30 & 38.42 & 4.54 & & & 38.08 & & & & 38.70 & 39.44 & 4.22 \\
\hline 12007085 & 7.60 & 1.17 & & & & & 7.89 & & & & & & \\
\hline 12007304 & 208.10 & 16.77 & 216.84 & 16.70 & 221.27 & 16.62 & 216.51 & 16.64 & & & & & 16.83 \\
\hline 12008680 & 24.30 & 3.68 & & & & & 24.52 & 3.79 & 22.72 & 2.73 & & & \\
\hline 12008797 & 31.70 & 4.35 & 31.90 & 4.00 & 31.54 & 4.15 & 32.10 & 4.04 & 28.59 & 4.00 & & & \\
\hline 12008916 & 160.40 & 12.97 & 160.46 & 12.55 & 161.35 & 12.99 & 159.71 & 12.85 & & & 161.70 & 165.38 & 13.00 \\
\hline 12012082 & 27.50 & 3.29 & 28.94 & 3.25 & 29.01 & 3.27 & 27.81 & 3.31 & & & 29.22 & 31.05 & 3.27 \\
\hline 12012725 & 25.90 & 3.32 & 26.09 & 3.37 & 25.63 & 3.14 & 25.96 & 3.33 & 25.74 & 3.32 & 26.35 & 27.62 & 3.29 \\
\hline 12016555 & 2.30 & 0.57 & & & & & & & & & & & \\
\hline 12020797 & 4.50 & 0.83 & & & & & & & & & & & \\
\hline 12021906 & 41.90 & 4.52 & 41.14 & 4.58 & 40.38 & 4.47 & 42.06 & 4.60 & & & 41.23 & 42.12 & 4.51 \\
\hline 12058556 & 105.50 & 9.29 & 104.50 & 9.35 & 104.67 & 9.34 & 105.03 & 9.29 & & & 106.01 & 111.04 & 9.32 \\
\hline 12060088 & 19.80 & 2.58 & 20.22 & 2.58 & 19.22 & & 19.69 & 2.49 & & & 20.19 & 21.69 & \\
\hline 12061622 & 33.20 & 4.19 & 32.94 & 4.20 & 34.53 & 4.06 & 33.11 & 4.20 & & & 34.68 & 37.36 & 4.13 \\
\hline 12066292 & 4.80 & 0.86 & & & & & & & & & & & \\
\hline 12070114 & 40.50 & 4.38 & 39.32 & 4.61 & 39.32 & 4.29 & 41.70 & 4.30 & 37.11 & & 40.03 & 41.25 & 4.35 \\
\hline 12101990 & 30.00 & 3.93 & 29.73 & 4.00 & 29.11 & 4.00 & 30.09 & & & & 30.08 & 31.16 & 4.01 \\
\hline 12102923 & 11.00 & 1.45 & & & & & 11.19 & 1.54 & & & & & \\
\hline 12104584 & 27.40 & 3.44 & 27.79 & 3.50 & & & 27.77 & 3.49 & & & & & \\
\hline 12109388 & 40.20 & 4.27 & 40.68 & 4.08 & 40.12 & 4.31 & 40.31 & 4.35 & 37.46 & & 40.69 & 42.20 & 4.25 \\
\hline 12109888 & 15.40 & 2.27 & 15.62 & 2.27 & 15.03 & 2.29 & 15.47 & 2.32 & & & & & \\
\hline 12110266 & 40.80 & 4.19 & 41.28 & 4.24 & 40.20 & 4.19 & 41.19 & 4.14 & 42.06 & & 41.44 & 41.61 & 4.21 \\
\hline 12116393 & 61.30 & 6.25 & 61.30 & 6.24 & 60.84 & 6.25 & 61.19 & 6.32 & & & 61.31 & 62.50 & 6.25 \\
\hline 12121207 & 26.90 & 3.72 & 26.98 & 3.68 & & & 27.29 & 3.60 & & & 27.25 & 28.79 & 3.56 \\
\hline 12152850 & 31.30 & 4.12 & 31.83 & 4.14 & 30.90 & 4.14 & 31.47 & 3.98 & 31.18 & 4.17 & 32.12 & 34.06 & 4.04 \\
\hline 12159715 & 62.50 & 6.49 & 63.55 & 6.38 & & & 62.53 & 6.42 & & & 63.69 & 64.20 & 6.45 \\
\hline 12168406 & 45.20 & 4.84 & 45.72 & 4.69 & 44.98 & 4.77 & 45.52 & 4.74 & & & 45.72 & 47.05 & 4.78 \\
\hline 12169845 & 31.30 & 3.32 & 30.12 & 3.30 & 29.57 & 3.11 & 31.65 & 3.31 & 25.76 & & 31.19 & 31.20 & 3.33 \\
\hline 12203243 & 192.60 & 15.30 & 194.24 & 15.15 & 200.00 & 15.07 & 197.30 & 15.17 & & & & & 15.10 \\
\hline 12208273 & 21.20 & 3.18 & 21.91 & 3.03 & & & 21.21 & 3.01 & & & & & \\
\hline 12217239 & 26.00 & 3.15 & 26.22 & 3.24 & & & 26.18 & 3.24 & & & 26.36 & 28.16 & 3.15 \\
\hline 12255028 & 37.10 & 4.24 & 37.52 & 4.10 & 36.47 & 4.22 & 37.54 & & & & 37.62 & 39.35 & 4.18 \\
\hline 12301444 & 3.40 & 0.99 & & & & & & & 2.19 & 0.57 & & & \\
\hline 12302104 & 34.50 & 4.17 & 34.78 & 4.32 & 34.15 & & 34.82 & & & & 34.75 & 36.22 & 4.07 \\
\hline 12302516 & 32.20 & 3.97 & 32.03 & 3.97 & 31.36 & & 32.66 & 4.08 & & & 32.55 & 34.03 & 3.92 \\
\hline 12306695 & 33.50 & 3.74 & 33.64 & 3.81 & 33.23 & 3.75 & 33.80 & 3.78 & & & 33.96 & 35.33 & 3.74 \\
\hline 12306763 & 8.50 & 1.36 & & & & & 8.49 & 1.36 & & & & & \\
\hline 12314927 & 14.00 & 1.93 & & & 13.97 & 1.90 & 14.28 & 1.98 & & & & & \\
\hline 12316494 & 30.20 & 3.76 & 31.13 & 3.86 & 31.80 & 3.89 & 30.24 & 3.74 & & & & & \\
\hline 12352536 & 14.70 & 2.22 & & & & & 14.79 & & & & & & \\
\hline 12366461 & 43.40 & 4.55 & 43.05 & 4.58 & 43.14 & 4.57 & 43.70 & 4.62 & 45.62 & & 43.38 & 44.10 & 4.56 \\
\hline 12454201 & 38.90 & 4.38 & 39.70 & 4.27 & 38.07 & 4.35 & 39.37 & 4.31 & 30.50 & 4.20 & 39.37 & 38.31 & 4.37 \\
\hline 12455504 & 37.70 & 3.95 & 38.00 & 3.90 & 35.72 & 3.83 & 37.31 & 3.93 & & & 38.02 & 38.85 & 3.92 \\
\hline 12458003 & 37.10 & 3.66 & 36.93 & 3.84 & 33.60 & 3.36 & 37.24 & 3.84 & & & 36.92 & 36.51 & 3.71 \\
\hline 12470054 & 164.10 & 12.73 & 161.50 & 12.56 & 164.27 & 12.50 & 163.53 & 12.66 & & & 160.95 & 162.28 & 12.64 \\
\hline 12506577 & 18.40 & 2.52 & 18.68 & 2.53 & 18.59 & 2.54 & 18.78 & & & & 18.89 & 19.58 & \\
\hline 12507577 & 81.70 & 7.55 & 81.32 & 7.55 & 82.05 & 7.59 & 81.80 & 7.54 & & & 82.27 & 84.66 & 7.56 \\
\hline 12519768 & 23.70 & 3.10 & 23.60 & 3.06 & 23.30 & 3.18 & 24.02 & 3.16 & & & 24.03 & 24.25 & \\
\hline 12520106 & 41.00 & 4.30 & 41.34 & 4.30 & 41.64 & 4.22 & 41.17 & 4.27 & & & 41.81 & 43.02 & 4.29 \\
\hline 12555458 & & & & & & & 34.32 & & & & & & \\
\hline 12555902 & 39.10 & 4.07 & 38.43 & 4.30 & & & 39.10 & 4.14 & & & 39.03 & 38.81 & 4.60 \\
\hline 12599612 & 52.00 & 5.46 & 52.21 & 5.43 & 52.09 & 5.33 & 51.54 & 5.41 & & & 52.51 & 53.66 & 5.31 \\
\hline 12599878 & 37.30 & 4.09 & 36.35 & 4.46 & 37.07 & 4.14 & 37.36 & 4.00 & & & 37.10 & 38.67 & \\
\hline 12601771 & & & & & 170.00 & 14.12 & 177.33 & 14.39 & & & 180.23 & 187.99 & 14.44 \\
\hline 12603035 & 29.70 & 4.02 & 29.10 & 3.97 & & & 29.89 & 3.89 & & & 29.53 & 30.60 & 4.10 \\
\hline 12688781 & 43.70 & 4.55 & 44.62 & 4.60 & 43.88 & 4.67 & 43.52 & 4.48 & & & 44.62 & 46.14 & 4.62 \\
\hline 12691122 & 30.60 & 3.88 & & & 29.58 & 3.92 & 30.90 & & & & 30.43 & 30.91 & 3.89 \\
\hline 12691734 & 42.50 & 4.32 & & & 47.10 & 4.42 & 42.65 & 4.22 & & & 46.10 & 47.74 & 4.30 \\
\hline 12784683 & 27.80 & 3.95 & & & 27.96 & 2.86 & 28.58 & 3.85 & & & & & \\
\hline 12884274 & 45.50 & 4.55 & & & & & & & & & & & \\
\hline
\end{tabular}

